\title{
Nuclear Activity in Nearby Galaxies
}

\author{
Luis C. Ho \\ The Observatories of the Carnegie Institution of Washington, 813 Santa Barbara St., \\ Pasadena, CA 91101; e-mail: lho@ociw.edu
}

KEYWORDS: accretion disks, active galactic nuclei, black holes, LINERs, radio galaxies, Seyfert galaxies

\begin{abstract}
A significant fraction of nearby galaxies show evidence of weak nuclear activity unrelated to normal stellar processes. Recent high-resolution, multiwavelength observations indicate that the bulk of this activity derives from black hole accretion with a wide range of accretion rates. The low accretion rates that typify most low-luminosity active galactic nuclei induce significant modifications to their central engine. The broad-line region and obscuring torus disappear in some of the faintest sources, and the optically thick accretion disk transforms into a three-component structure consisting of an inner radiatively inefficient accretion flow, a truncated outer thin disk, and a jet or outflow. The local census of nuclear activity supports the notion that most, perhaps all, bulges host a central supermassive black hole, although the existence of active nuclei in at least some late-type galaxies suggests that a classical bulge is not a prerequisite to seed a nuclear black hole.
\end{abstract}

\section{CONTENTS}

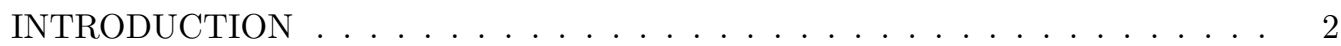

SPECTRAL CLASSIFICATION OF GALACTIC NUCLEI . . . . . . . . . . . 4

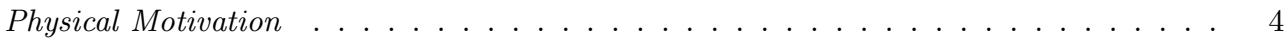

Sample Spectra . . . . . . . . . . . . . . . . . . . . . . . 5

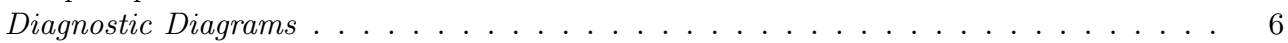

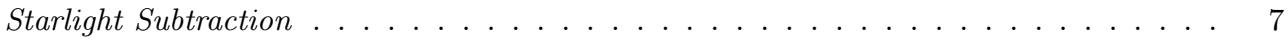

Other Classification Criteria . . . . . . . . . . . . . . . 8

SURVEYS OF NEARBY GALACTIC NUCLEI . . . . . . . . . . . . . . . . . . 10

The Palomar Survey . . . . . . . . . . . . . . . . . . . . 10

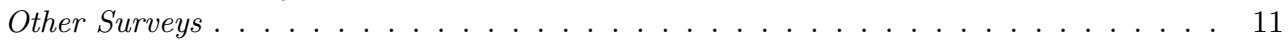

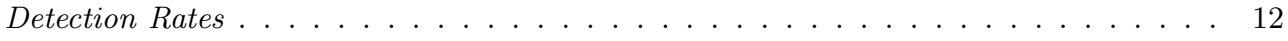

Broad Emission Lines . . . . . . . . . . . . . . . . . . . . . . . . . . . . . . . . . 14

Robustness and Completeness . . . . . . . . . . . . . . . . 15

HOST GALAXY PROPERTIES . . . . . . . . . . . . . . . . . . . 16

Global Parameters . . . . . . . . . . . . . . . . . . . . 16

Nuclear Stellar Populations . . . . . . . . . . . . . . . . . . . . . . . . . . . 16

Influence of Bars and Environment . . . . . . . . . . . . . . . . . . . . . . . . . 18

NUCLEAR PROPERTIES . . . . . . . . . . . . . . . . . . . . . . . 18

Ionizing Continuum Radiation . . . . . . . . . . . . . . . . . . . . . 18

Radio Cores . . . . . . . . . . . . . . . . . . . . . . 20 20

X-ray Cores . . . . . . . . . . . . . . . . . . . . . . . . . 22 
Circumnuclear Thermal Plasma . . . . . . . . . . . . . . . . 25

Broad-line Region . . . . . . . . . . . . . . . . . . . . . . 25

Torus . . . . . . . . . . . . . . . . . . . . . . . . 29

Narrow-line Region Kinematics . . . . . . . . . . . . . . . . . . . . . . . . 30

Spectral Energy Distribution . . . . . . . . . . . . . . . . . . . 31

Luminosity Function . . . . . . . . . . . . . . . . . . . . . . . . . . . . . . . . . . . 35

Bolometric Luminosities and Eddington Ratios . . . . . . . . . . . . . . . . . . . . . . 36

EXCITATION MECHANISMS . . . . . . . . . . . . . . . . . . . 38

Nonstellar Photoionization . . . . . . . . . . . . . . . . . 38

Contribution from Fast Shocks . . . . . . . . . . . . . . . . . . . . 40

Contribution from Stellar Photoionization . . . . . . . . . . . . . . 41

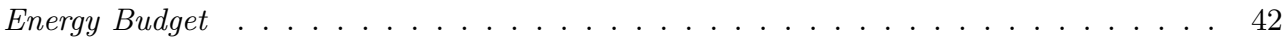

The Nature of Transition Objects and a Unified View of LLAGNs . . . . . . . . . . . . 44

IMPLICATIONS FOR BLACK HOLE DEMOGRAPHICS . . . . . . . . . . . . . 47

IMPLICATIONS FOR ACCRETION PHYSICS . . . . . . . . . . . . . . . . 50

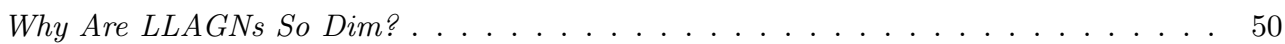

The Disk-Jet Connection . . . . . . . . . . . . . . . . . . . . . . . . 54

The Central Engine of LLAGNs . . . . . . . . . . . . . . . . . . 57

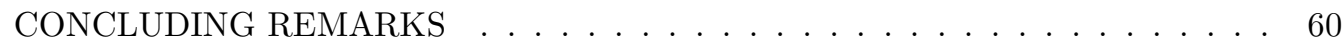

\section{INTRODUCTION}

Far from being rare, exotic entities that inhabit only a tiny fraction of galaxies, central black holes (BHs) are now believed to be basic constituents of most, if not all, massive galaxies (Magorrian et al. 1998; Kormendy 2004). Although less common in low-mass systems, central BHs also have been identified in some late-type, even dwarf, galaxies (Filippenko \& Ho 2003; Barth et al. 2004; Greene \& Ho 2004, 2007b; Dong et al. 2007; Greene, Ho \& Barth 2008). The realization that BH mass correlates strongly with the properties of the host galaxy (Kormendy 1993; Kormendy \& Richstone 1995; Magorrian et al. 1998; Gebhardt et al. 2000; Ferrarese \& Merritt 2000; Barth, Greene \& Ho 2005; Greene \& Ho 2006) has generated intense interest in linking BH growth with galaxy formation, as attested by the increasing number of conferences focusing on this theme (e.g., Schmitt, Kinney \& Ho 1999; Ho 2004a; Storchi-Bergmann, Ho \& Schmitt 2004; Merloni, Nayakshin \& Sunyaev 2005; Fiore 2006). As a direct manifestation of BH accretion, and therefore $\mathrm{BH}$ growth, active galactic nuclei (AGNs) and the consequences of their energy feedback have figured prominently in most current ideas of structure formation (e.g., Granato et al. 2004; Springel, Di Matteo \& Hernquist 2005; Hopkins et al. 2006). At the same time, the community's heightened awareness of the importance of BHs has galvanized broad interest in the study of the AGN phenomenon itself. With the BH mass known-arguably the most fundamental parameter of the system - what once rested on phenomenological analysis can now be put on a more secure physical basis.

This review focuses on nuclear activity in nearby galaxies. By selection, most of the objects occupy the faintest end of the AGN luminosity function and have very low accretion rates. While energetically unimpressive, low-luminosity AGNs (LLAGNs) deserve scrutiny for several reasons. By virtue of the short duty cycle of $\mathrm{BH}$ accretion $\left(\sim 10^{-2}\right.$; Greene \& Ho 2007a), most AGNs spend their lives in a low state, such that the bulk of the population has relatively modest luminosities. 
Over the past several decades, this attribute has led to considerable controversy regarding the physical origin of LLAGNs. As absolute luminosity can no longer be used as a defining metric of nonstellar activity, many alternative excitation mechanisms have been proposed to explain LLAGNs. Fortunately, the advent of new telescopes and new analysis techniques have yielded many fresh insights into this thorny old problem. A major goal of this review is to summarize these recent developments. Along the way, I will emphasize how the collective properties of LLAGNs can shed light on a poorly understood regime of the central engine, namely that governed by low mass accretion rate. A key point I will stress is that LLAGNs are not simply scaled-down versions of their more familiar cousins, the classical Seyfert galaxies and quasars.

Despite the impressive progress made in the direct detection of central BHs in nearby inactive galaxies, our knowledge of the demographics of BHs remains highly incomplete. Direct measurements of $\mathrm{BH}$ masses based on resolved gas or stellar kinematics are still far from routine and are available only for about three dozen galaxies. Certainly nothing approaching a "complete" sample exists yet. More importantly, it is not obvious that the current statistics are unbiased. As discussed by Barth (2004), most nearby galaxies possess chaotic gas velocity fields that defy simple analysis. Stellar kinematics provide a powerful alternative, but in practice this technique has been limited to relatively dust-free systems and, for practical reasons, to galaxies of relatively high central surface brightness. The latter restriction selects against the most luminous, giant ellipticals. Present surveys also severely underrepresent disk-dominated galaxies, because the bulge component in these systems is inconspicuous and star formation tends to perturb the velocity field of the gas. Finally, apart from galaxies within the Local Group, even the highest angular resolution currently achieved is inadequate to directly detect BHs with masses $\lesssim 10^{6} M_{\odot}$. Consequently we are nearly completely ignorant about the low end of the $\mathrm{BH}$ mass function. Given the above limitations, it is desirable to consider alternative constraints on BH demography. The commonly held and now well-substantiated premise that AGNs derive their energy output from $\mathrm{BH}$ accretion implies that an AGN signifies the presence of a central $\mathrm{BH}$ in a galaxy. The AGN signature in and of itself provides little direct information on BH masses, but AGN statistics can inform us, effectively and efficiently, of some key aspects of BH demography. For example, what fraction of all galaxies contain BHs? Do BHs exist preferentially in galaxies of certain types? Does environment matter? I will discuss how studies of nearby AGNs have begun to answer some of these important questions.

This review is structured as follows. I begin with an overview of the basic methodology of the spectral classification of emission-line nuclei ( $(2)$ by describing the currently adopted system, its physical motivation, the complications of starlight subtraction, and some practical examples. Section 3 summarizes past and current spectroscopic surveys and introduces the Palomar survey, covering detection rates, measurement of weak broad emission lines, and issues of robustness and completeless. Host galaxy properties are the subject of $\S 4$, where in addition to global and environmental effects I also cover results on nuclear stellar populations. In Section 5, I devote considerable attention to the nuclear properties of LLAGNs in general and LINERs in particular, focusing on modern results obtained from high-resolution, multiwavelength observations from radio to hard-X-ray energies. I use these data to draw inferences concerning the broadline region (BLR), torus, narrow-line region (NLR), spectral energy distribution 
(SED), luminosity function, bolometric luminosities, and Eddington ratios. This section contains many technical details, but these will be essential ingredients for formulating the big picture at the end. Section 6 covers the controversial subject of the excitation mechanism of LINERs, the growing puzzle concerning the energy budget in these systems, and the nature of narrow-line nuclei. The implications of LLAGNs for BH demographics are discussed in Section 7. Section 8 attempts to synthesize the disparate lines of evidence into a coherent physical framework for LLAGNs and their relation to other classes of objects. Finally, Section 9 concludes with some personal perspectives and suggestions for future directions.

\section{SPECTRAL CLASSIFICATION OF GALACTIC NUCLEI}

\subsection{Physical Motivation}

AGNs can be identified by a variety of methods. Most AGN surveys rely on some aspect of the distinctive AGN spectrum, such as the presence of strong or broad emission lines, an unusually blue continuum, or strong radio or X-ray emission. All of these techniques are effective, but none is free from selection effects. To search for AGNs in nearby galaxies, where the nonstellar signal of the nucleus is expected to be weak relative to the host galaxy, the most effective and least biased method is to conduct a spectroscopic survey of a complete, opticalflux limited sample of galaxies. To be sensitive to weak emission lines, the survey must be deep and of sufficient spectral resolution. To obtain reliable line intensity ratios on which the principal nuclear classifications are based, the data must have accurate relative flux calibration, and one must devise a robust scheme to correct for the starlight contamination.

The most widely used system of spectral classification of emission-line nuclei follows the method promoted by Baldwin, Phillips \& Terlevich (1981), and later modified by Veilleux \& Osterbrock (1987). The basic idea is that the relative strengths of certain prominent emission lines can be used to probe the nebular conditions of a source. In the context of the present discussion, the most important diagnostic is the source of excitation, which broadly falls into two categories: stellar photoionization or photoionization by a centrally located, spectrally hard radiation field, such as that produced by the accretion disk of a massive BH. How does one distinguish between the two? The forbidden lines of the doublet [O I] $\lambda \lambda 6300,6364$ arise from collisional excitation of $\mathrm{O}^{0}$ by hot electrons. Since the ionization potential of $\mathrm{O}^{0}(13.6 \mathrm{eV})$ is nearly identical to that of hydrogen, in an ionization-bounded nebula [O I] is produced predominantly in the "partially ionized zone," wherein both neutral oxygen and free electrons coexist. In addition to $\mathrm{O}^{0}$, the conditions of the partially ionized zone are also favorable for $\mathrm{S}^{+}$and $\mathrm{N}^{+}$, whose ionization potentials are $23.3 \mathrm{eV}$ and $29.6 \mathrm{eV}$, respectively. Hence, in the absence of abundance anomalies, [N II] $\lambda \lambda 6548,6583$ and [S II] $\lambda \lambda 6716,6731$ are strong (relative to, say, $\mathrm{H} \alpha$ ) whenever $[\mathrm{O} \mathrm{I}]$ is strong, and vice versa.

In a nebula photoionized by young, massive stars, the partially ionized zone is very thin because the ionizing spectrum of OB stars contains few photons with energies greater than $13.6 \mathrm{eV}$. Hence, in the optical spectra of H II regions and starburst nuclei (hereinafter H II nuclei ${ }^{1}$ ) the low-ionization transitions [N II], [S II], and especially [O I] are very weak. By contrast, a harder radiation field, such as

\footnotetext{
${ }^{1}$ As originally defined (Weedman et al. 1981), a starburst nucleus is one whose current star
} 


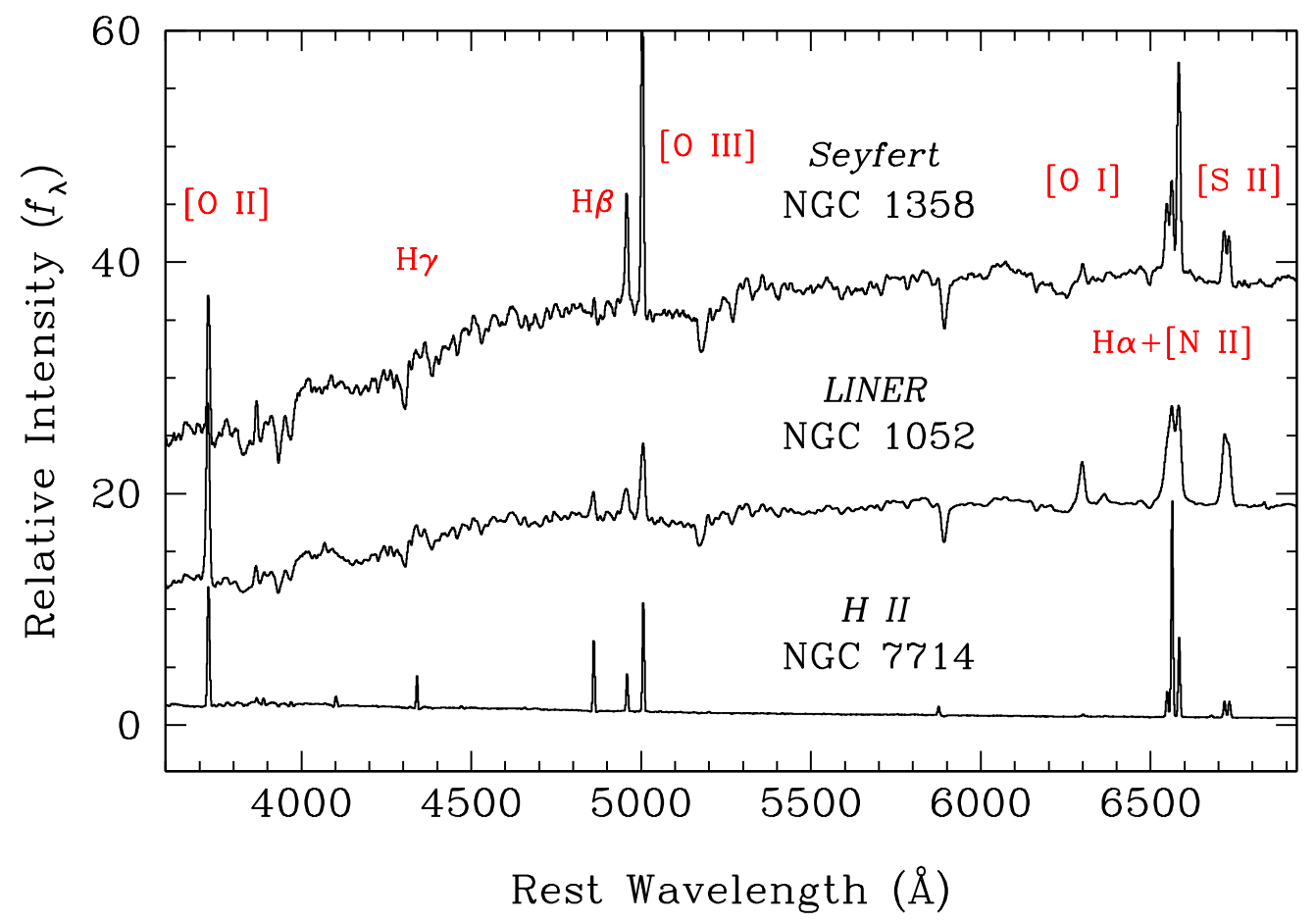

Figure 1: Sample optical spectra of the various classes of emission-line nuclei. Prominent emission lines are identified. (Based on Ho, Filippenko \& Sargent 1993 and unpublished data.)

that of an AGN power-law continuum that extends into the extreme-ultraviolet (UV) and X-rays, penetrates much deeper into an optically thick cloud, creating an extensive partially ionized zone and hence strong low-ionization forbidden lines. A hard AGN radiation field also boosts the production of collisionally excited forbidden line emission because its high thermal energy deposition rate enhances the gas temperature.

\subsection{Sample Spectra}

The spectra shown in Figure 1 illustrate the empirical distinction between AGNs and H II nuclei. In NGC 7714, which has a well-known starburst nucleus (Weedman et al. 1981), [O I], [N II], and [S II] are weak relative to $\mathrm{H} \alpha$. The [O III] $\lambda \lambda 4959,5007$ doublet is quite strong compared to [O II] $\lambda 3727$ or $\mathrm{H} \beta$ because the metal abundance of NGC 7714's nucleus is rather low, although the ionization level of H II nuclei can span a wide range, depending on metallicity (Ho, Filippenko \& Sargent 1997c; Kewley et al. 2001; Groves, Heckman \& Kauffmann 2006). On the other hand, the low-ionization lines are markedly stronger in the other two objects shown, both of which qualify as AGNs. NGC 1358 has a "high-ionization" AGN or "Seyfert" nucleus. NGC 1052 is the prototype of the

formation rate is much higher than its past average rate. Since in general we do not know the star formation history of any individual object, I will adopt the more general designation of "H II nucleus." 

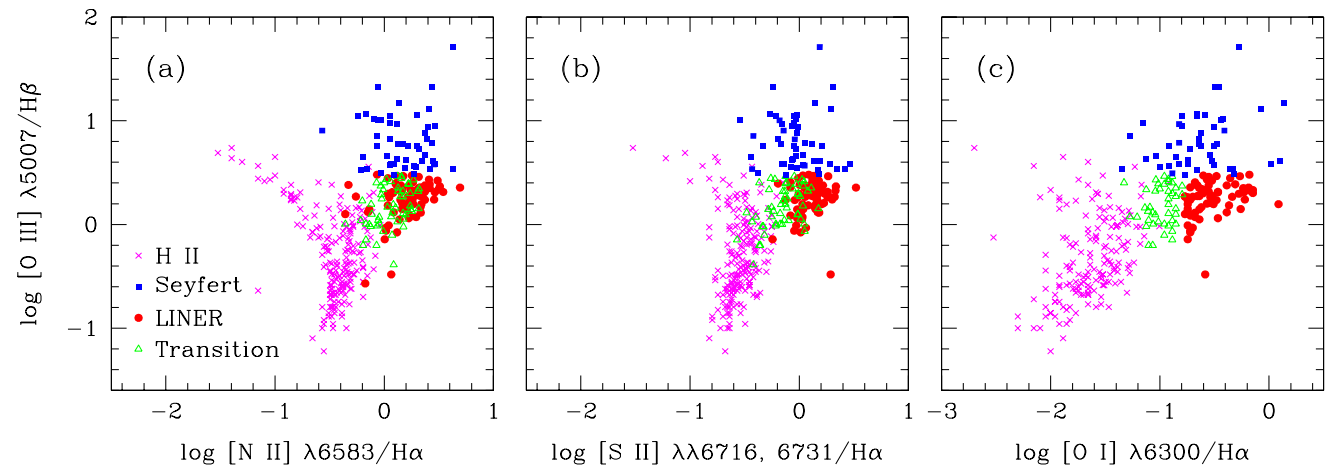

Figure 2: Diagnostic diagrams plotting (a) $\log [\mathrm{O}$ III $] \lambda 5007 / \mathrm{H} \beta$ versus $\log [\mathrm{N} \mathrm{II}]$ $\lambda 6583 / \mathrm{H} \alpha,(b) \log [\mathrm{O}$ III $] \lambda 5007 / \mathrm{H} \beta$ versus $\log [\mathrm{S} \mathrm{II}] \lambda \lambda 6716,6731 / \mathrm{H} \alpha$, and $(c)$ $\log [\mathrm{O}$ III $] \lambda 5007 / \mathrm{H} \beta$ versus $\log [\mathrm{O} \mathrm{I}] \lambda 6300 / \mathrm{H} \alpha$. (Adapted from Ho, Filippenko \& Sargent 1997a.)

class known as "low-ionization nuclear emission-line regions" or LINERs (Heckman 1980b). The ionization level can be judged by the relative strengths of the oxygen lines, but in practice is most easily gauged by the $[\mathrm{O}$ III $] / \mathrm{H} \beta$ ratio. In the commonly adopted system of Veilleux \& Osterbrock (1987), the division between Seyferts and LINERs occurs at [O III] $\lambda 5007 / \mathrm{H} \beta=3.0$. Ho, Filippenko \& Sargent (2003) stress, however, that this boundary has no strict physical significance. The ionization level of the NLR in large, homogeneous samples of AGNs spans a wide and apparently continuous range; there is no evidence for any clearcut transition between Seyferts and LINERs (Ho, Filippenko \& Sargent 2003), although with sufficient numbers, the two classes do delineate two distinct loci in optical diagnostic diagrams (Kewley et al. 2006).

The classification system discussed above makes no reference to the profiles of the emission lines. Luminous AGNs such as quasars and many classical Seyfert galaxies exhibit permitted lines with a characteristically broad component, with full width at half-maximum (FWHM) widths of $\sim 1000$ to $10,000 \mathrm{~km} \mathrm{~s}^{-1}$. This component arises from the BLR, which is thought to be physically distinct from the NLR responsible for the narrow lines. Following Khachikian \& Weedman (1974), it is customary to refer to Seyferts with and without (directly) detectable broad lines as "type 1" and "type 2" sources, respectively. As discussed in $\S 3.4$, this nomenclature can also be extended to include LINERs.

\subsection{Diagnostic Diagrams}

The classification system of Veilleux \& Osterbrock (1987), which I adopt throughout this paper, is based on two-dimensional line-intensity ratios constructed from [O III] $\lambda 5007, \mathrm{H} \beta \lambda 4861,[\mathrm{O} \mathrm{I}] \lambda 6300, \mathrm{H} \alpha \lambda 6563,[\mathrm{~N} \mathrm{II}] \lambda 6583$, and [S II] $\lambda \lambda 6716$, 6731 (here $\mathrm{H} \beta$ and $\mathrm{H} \alpha$ refer only to the narrow component of the line). The main virtues of this system, shown in Figure 2, are (1) that it uses relatively strong lines, (2) that the lines lie in an easily accessible region of the optical spectrum, and (3) that the line ratios are relatively insensitive to reddening corrections because of the close separation of the lines. The definitions of the various 
classes of emission-line objects are given in Ho, Filippenko \& Sargent (1997a) ${ }^{2}$. In addition to the three main classes discussed thus far-H II nuclei, Seyferts, and LINERs - Ho, Filippenko \& Sargent (1993) identified a group of "transition objects" whose [O I] strengths are intermediate between those of $\mathrm{H}$ II nuclei and LINERs. Since they tend to emit weaker [O I] emission than classical LINERs, previous authors have called them "weak-[O I] LINERs" (Filippenko \& Terlevich 1992; Ho \& Filippenko 1993). Ho, Filippenko \& Sargent (1993) postulated that transition objects are composite systems having both an H II region and a LINER component; I will return to the nature of these sources in $\S 6.5$.

Note that my definition of LINERs differs from that originally proposed by Heckman (1980b), who used solely the oxygen lines: [O II] $\lambda 3727>[\mathrm{O}$ III] $\lambda 5007$ and [O I] $\lambda 6300>0.33$ [O III] $\lambda 5007$. The two definitions, however, are nearly equivalent. Inspection of the full optical spectra of Ho, Filippenko \& Sargent (1993), for example, reveals that emission-line nuclei classified as LINERs based on the Veilleux \& Osterbrock diagrams almost always also satisfy Heckman's criteria. This is a consequence of the inverse correlation between $[\mathrm{O}$ III $] / \mathrm{H} \beta$ and $[\mathrm{O}$ II $] /[\mathrm{O}$ III $]$ in photoionized gas with fairly low excitation.

\subsection{Starlight Subtraction}

The scheme described above, while conceptually simple, overlooks one key practical complication. The integrated spectra of galactic nuclei include starlight, which in most nearby systems overwhelms the nebular line emission (Figure 1). Any reliable measurement of the emission-line spectrum of galactic nuclei, therefore, must properly account for the starlight contamination.

An effective strategy for removing the starlight from an integrated spectrum is that of "template subtraction," whereby a template spectrum devoid of emission lines is suitably scaled to and subtracted from the spectrum of interest to yield a continuum-subtracted, pure emission-line spectrum. A number of approaches have been adopted to construct the template. These include using (1) the spectrum of an off-nuclear position within the same galaxy (e.g., Storchi-Bergmann, Baldwin \& Wilson 1993); (2) the spectrum of a different galaxy devoid of emission lines (e.g., Costero \& Osterbrock 1977; Filippenko \& Halpern 1984; Ho, Filippenko \& Sargent 1993); (3) a weighted linear combination of the spectra of a number different galaxies, chosen to best match the stellar population and velocity dispersion (Ho, Filippenko \& Sargent 1997a); (4) a variant of (3), but employing a stellar library and simultaneously fitting for the emission lines and accounting for dust reddening (Sarzi et al. 2007); (5) a mean spectrum derived from a principal-component analysis of a large set of galaxies (Hao et al. 2005a); and (6) a model spectrum constructed from population synthesis techniques, using as input a library of spectra of either individual stars (e.g., Keel 1983c), synthesis models (e.g., Tremonti et al. 2004; Sarzi et al. 2005), or star clusters (e.g., Bonatto, Bica \& Alloin 1989). Some studies (e.g., Kim et al. 1995) implicitly assume that only the hydrogen Balmer lines are contaminated by starlight and that the absorption-line component can be removed by assuming a constant equivalent width ( $\mathrm{EW}=2-3 \AA)$. This procedure is inadequate for a number of reasons. First, the stellar population of nearby galactic nuclei, although relatively

\footnotetext{
${ }^{2}$ The classification criteria adopted here differ slightly from those proposed by Kewley et al. (2001), Kauffmann et al. (2003), or Stasińska et al. (2006), but this difference has little effect on the general conclusions.
} 


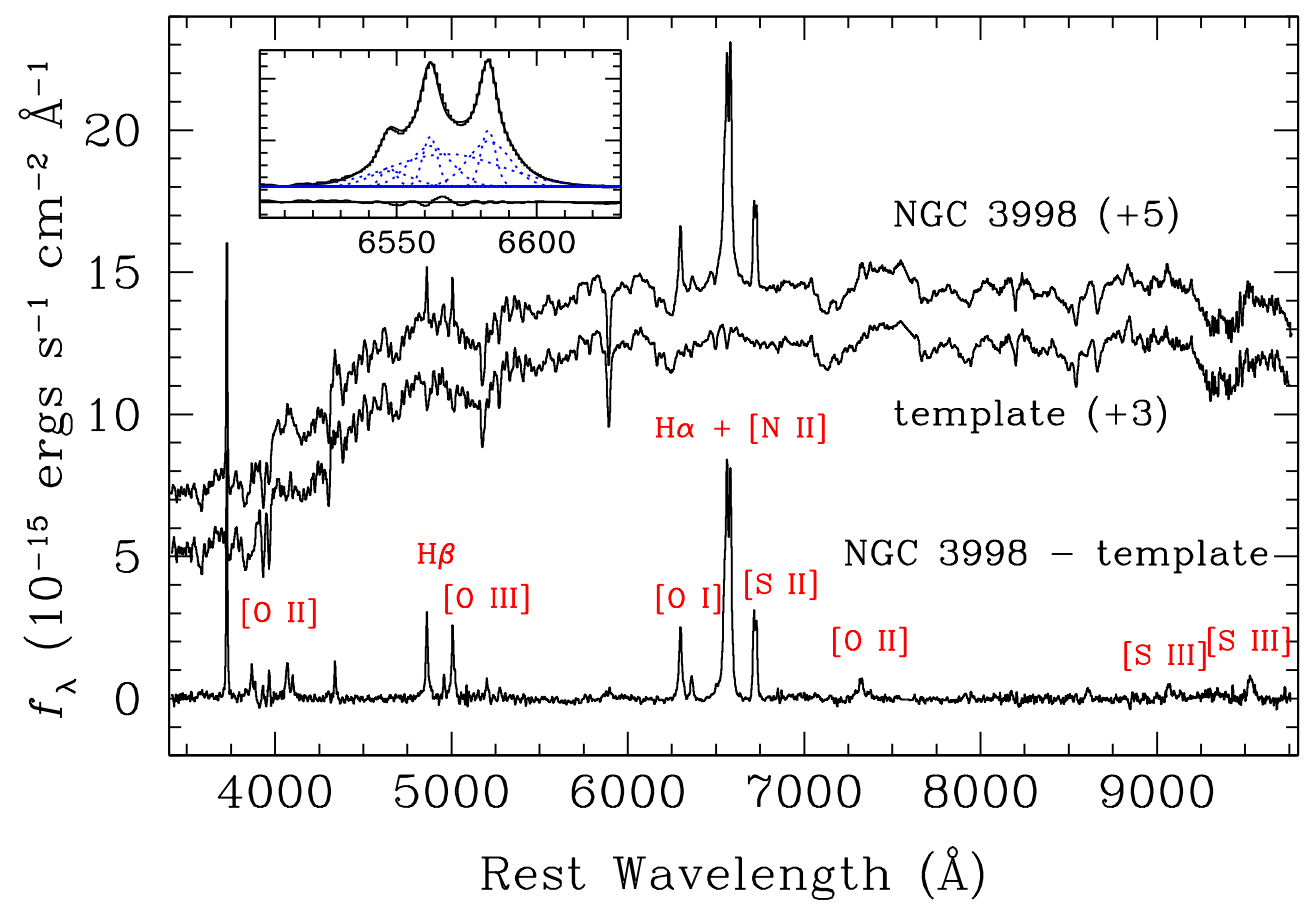

Figure 3: Illustration of starlight removal for NGC 3998 using the template galaxy NGC 3115. Prominent emission lines are labeled. The insert shows an expanded view of the $\mathrm{H} \alpha+[\mathrm{N} \mathrm{II}]$ region and a multi-Gaussian decomposition leading to the detection of a broad $\mathrm{H} \alpha$ component. (Adapted from Ho, Filippenko \& Sargent 1993 and Ho et al. 1997e.)

uniform, is by no means invariant (Ho, Filippenko \& Sargent 2003). Second, the equivalent widths of the different Balmer absorption lines within each galaxy are generally not constant. Third, the Balmer absorption lines affect not only the strength but also the shape of the Balmer emission lines. And finally, starlight contaminates more than just the Balmer lines.

Figure 3 illustrates the starlight subtraction process for the LINER NGC 3998. Note that in the original observed spectrum, many of the weaker emission lines were hardly visible, whereas after starlight subtraction, they can easily be measured. The intensities of even strong lines such $\mathrm{H} \beta$ and [O III] $\lambda \lambda 4959,5007$ are modified. Importantly, starlight correction is essential for properly identifying the weak broad $\mathrm{H} \alpha$ component in NGC 3998.

\subsection{Other Classification Criteria}

Although the traditional optical classification system described above is the most widely used, there are instances when features in other spectral regions may be more practical or useful. Surveys of intermediate-redshift galaxies, for example, cannot routinely access the $\mathrm{H} \alpha$ region, and under such circumstances it is desirable to devise a classification system based only on the blue part of the spectrum. Diagnostic diagrams proposed by Rola, Terlevich \& Terlevich (1997) 
based on the strong lines [O II] $\lambda 3727$, [Ne III] $\lambda \lambda 3869,3968, \mathrm{H} \beta$, and [O III] $\lambda \lambda 4959,5007$ provide moderately effective discrimination between starbursts and AGNs. A number of redshift surveys have searched for narrow-line AGNs based on the presence of [Ne V] $\lambda \lambda 3346,3426$ (e.g., Hall et al. 2000; Barger et al. 2001; Szokoly et al. 2004). With an ionization potential of $97 \mathrm{eV}$, [Ne V] unambiguously arises from nonstellar excitation, but the practical difficulty is that these lines are quite weak (strength $\sim 10 \%$ of [O III] $\lambda 5007$ ) and often can only be detected with confidence in stacked spectra (e.g., Zakamska et al. 2003).

The long-standing controversy over the relevance of shock excitation also has led to the development of line diagnostics outside of the traditional optical window. Díaz, Pagel \& Wilson (1985; see also Díaz, Pagel \& Terlevich 1985; Kirhakos \& Phillips 1989) suggested that [S III] $\lambda \lambda 9069$, 9532, in combination with the optical lines of [O II], [O III], and [S II], are effective in identifying shock-excited nebula. Since shock heating on average achieves higher equilibrium electron temperatures than photoionization, high-ionization UV lines such as [N V] $\lambda 1240$ and C IV $\lambda 1549$ can serve as a powerful discriminant between these two processes (e.g., Allen, Dopita \& Tsvetanov 1998). The limited availability of UV spectra, however, has restricted the wide use of these diagnostics.

Rapid progress in infrared (IR) technology has offered an important new window that is not only less affected by dust but also potentially has distinctive diagnostic power. Alonso-Herrero et al. (1997) show that Fe II 1.644/Br $\gamma$ can serve as an effective substitute for the conventional [O I] $\lambda 6300 / \mathrm{H} \alpha$ ratio. Unfortunately, other strong near-IR features, notably the vibrational lines of $\mathrm{H}_{2}$, are less useful because they can be excited by multiple mechanisms (Larkin et al. 1998). The mid-IR regime is much more promising, particularly with the sensitivity and wide bandpass afforded by Spitzer. For the first time, many of the diagnostic lines previously discussed in a theoretical context (Spinoglio \& Malkan 1992; Voit 1992) actually can now be measured (e.g., Bendo et al. 2006; Dale et al. 2006; Sturm et al. 2005, 2006; Rupke et al. 2007). In addition to highionization lines such as [Ne III] $\lambda 15.5 \mu \mathrm{m}$, [Ne V] $\lambda 14.3 \mu \mathrm{m}$, and [O IV] $\lambda 25.9 \mu \mathrm{m}$, the low-ionization transitions of Fe II $\lambda 26.0 \mu \mathrm{m}$ and [Si II] $\lambda 34.8 \mu \mathrm{m}$ may prove to be especially useful as they can constrain models of photo-dissociation and X-ray dissociation regions. The hard radiation field of AGNs, even of low-luminosity objects such as LINERs, appears to leave an imprint on the detailed emission spectrum of polycyclic aromatic hydrocarbons (Sturm et al. 2006; Smith et al. 2007).

Finally, a comment on nomenclature. It is important to stress that the classification scheme outlined above, physically motivated by the desire to separate objects by their source of excitation, is based strictly on the characteristics of the narrow emission lines and not on ancillary attributes such as luminosity, presence of broad emission lines, galaxy morphology, or radio properties. Although one still customarily draws a quaint distinction between quasars and Seyferts based on luminosity, it is widely acknowledged that this division is largely historical. In terms of their position on the line-ratio diagrams, quasars fall on the highionization branch and thus can be classified as Seyferts. The same holds for many broad-line and narrow-line radio galaxies, including most Fanaroff \& Riley (FR; 1974) type II radio sources, whose high luminosities generally translate directly into a high degree of ionization. By the same token, most FR I sources, because of their low luminosity, typically have fairly low-ionization spectra, and hence technically qualify as LINERs. FR I radio galaxies and LINERs are not separate 
beasts (cf. Falcke, Körding \& Markoff 2004; Chiaberge, Capetti \& Macchetto 2005). Strong historical prejudice also compels many to regard Seyfert nuclei as invariably radio-quiet sources that reside exclusively in spiral hosts, when, in fact, neither rule strictly holds (Ho \& Peng 2001). Despite claims to the contrary (Krolik 1998; Sulentic, Marziani \& Dultzin-Hacyan 2000), broad emission lines emphatically are not solely confined to Seyfert nuclei (§3.4). This misconception has led some people to define the Seyfert and LINER classes by their presence or absence of broad emission lines.

\section{SURVEYS OF NEARBY GALACTIC NUCLEI}

\subsection{The Palomar Survey}

The earliest redshift surveys already indicated that the spectra of galaxy centers often show strong emission lines (e.g., Humason, Mayall \& Sandage 1956). In many instances, the spectra revealed abnormal line-intensity ratios, most notably the unusually great strength of [N II] relative to $\mathrm{H} \alpha$ (Burbidge \& Burbidge 1962, 1965; Rubin, Ford \& Thonnard 1980; Rose \& Searle 1982). That the optical emission-line spectra of some nuclei show patterns of low ionization was noticed from time to time, primarily by Osterbrock and his colleagues (e.g., Osterbrock \& Dufour 1973; Osterbrock \& Miller 1975; Koski \& Osterbrock 1976; Costero \& Osterbrock 1977; Grandi \& Osterbrock 1978; Phillips 1979), but also by others (e.g., Disney \& Cromwell 1971; Danziger, Fosbury \& Penston 1977; Fosbury et al. 1977, 1978; Penston \& Fosbury 1978; Stauffer \& Spinrad 1979).

The activity in this field culminated in the 1980s, beginning with the recognition (Heckman, Balick \& Crane 1980; Heckman 1980b) of LINERs as a major constituent of the extragalactic population, and then followed by further systematic studies of larger samples of galaxies (Stauffer 1982a, 1982b; Keel 1983b, 1983c; Phillips et al. 1986; Véron \& Véron-Cetty 1986; Véron-Cetty \& Véron 1986; see Ho 1996 for more details). These surveys established three important results. (1) A large fraction of local galaxies contain emission-line nuclei. (2) Many of these sources are LINERs. And (3) LINERs may be accretion-powered systems.

Despite the successes of these seminal studies, there was room for improvement. Although most of the surveys attempted some form of starlight subtraction, the accuracy of the methods used was limited (see discussion in Ho, Filippenko \& Sargent 1997a), the procedure was sometimes inconsistently applied, and in some of the surveys, starlight subtraction was altogether neglected. The problem is exacerbated by the fact that the apertures used for the observations were quite large, thereby admitting an unnecessarily large amount of starlight. Furthermore, most of the data were collected with rather low spectral resolution (FWHM $\approx 10$ $\AA$ ). Besides losing useful kinematic information, blending between the emission and absorption components further compromises the ability to separate the two.

Thus, it was clear that much would be gained from a survey having greater sensitivity to the detection of emission lines. The sensitivity could be improved in at least four ways: by taking spectra with higher signal-to-noise ratio and spectral resolution, by using a narrower slit to better isolate the nucleus, and by employing more effective methods to handle the starlight correction.

The Palomar spectroscopic survey of nearby galaxies (Filippenko \& Sargent 1985; Ho, Filippenko \& Sargent 1995, 1997a, 1997b, 1997c, 1997d, 2003; Ho et 
al. 1997e) was designed with these goals in mind. Using a double CCD spectrograph mounted on the Hale 5-m reflector, high-quality, moderate-resolution, long-slit spectra were obtained for a magnitude-limited $\left(B_{T} \leq 12.5 \mathrm{mag}\right)$ sample of 486 northern $\left(\delta>0^{\circ}\right)$ galaxies. Drawn from the Revised Shapley-Ames (RSA) Catalog of Bright Galaxies (Sandage \& Tammann 1981), the bright magnitude limit ensured that the sample had a high degree of completeness. The spectra simultaneously cover the wavelength ranges $6210-6860 \AA$ with $\sim 2.5 \AA$ resolution (FWHM) and $4230-5110 \AA$ with $\sim 4 \AA$ resolution. Most of the observations were obtained with a narrow $\left(1^{\prime \prime}-2^{\prime \prime}\right)$ slit, and relatively long exposure times gave high signal-to-noise ratios. This survey still contains the largest database to date of homogeneous and high-quality optical spectra of nearby galaxies. It is also the most sensitive; the detection limit for emission lines is $\mathrm{EW} \approx 0.25 \AA$, roughly an order-of-magnitude improvement compared to previous or subsequent work. The selection criteria ensure that the sample gives a fair representation of the local $(z \approx 0)$ galaxy population, and the proximity of the objects (median distance $=$ $17 \mathrm{Mpc}$ ) results in relatively good spatial resolution (typically $\lesssim 200 \mathrm{pc})^{3}$. These properties of the Palomar survey make it ideally suited to address issues on the demographics and physical properties of nearby, and especially low-luminosity, AGNs. Unless otherwise noted, most of the results presented in this paper will be taken from the Palomar survey.

The Palomar survey has one other virtue that is not widely appreciated. Because the sample is large and essentially unbiased with respect to nuclear or global properties, it is ideally suited for comparative studies of various subpopulations. Examples include efforts to discern differences between type 1 versus type 2 sources to test AGN unification, to ascertain the influence of bars or environment on nuclear activity, or to test for subtle differences between the different AGN classes. The robustness of these and similar studies almost always hinges on the availability of proper control samples. With the Palomar survey, there is no need to construct a separate control sample, which is always a difficult and somewhat dubious undertaking, because the control sample is built into the survey.

\subsection{Other Surveys}

For completeness, I mention several other sources of nearby AGNs that have been widely used by the community. The AGN sample culled from the CfA Redshift Survey (Huchra \& Burg 1992) has been an important resource for a long time. Comprising 47 relatively bright Seyferts and a handful of LINERs, the CfA sample in many ways complements the Palomar sample at the bright end of the luminosity function. However, as discussed in Ho \& Ulvestad (2001), the selection effects of the CfA sample are not easy to quantify because of the subjective and somewhat nonstandard manner in which AGNs were picked from the parent survey. Prior to the full publication of the Palomar survey, Maiolino \& Rieke (1995) assembled a compilation of 91 Seyferts from a literature search of the galaxies in the RSA. These "RSA Seyferts" have subsequently been used in a number of follow-up studies. The substantial improvement in the data quality and analysis of the Palomar survey has resulted in many revised classifications of the RSA galaxies. Lastly, a cautionary note. Many investigators rely on

\footnotetext{
${ }^{3} \mathrm{~A}$ distance scale based on $H_{0}=75 \mathrm{~km} \mathrm{~s}^{-1} \mathrm{Mpc}^{-1}$ is assumed throughout
} 
literature compilations, such as those assembled in Véron-Cetty \& Véron's (2006) catalog or the NASA/IPAC Extragalactic Database, as their source for AGN classifications. This is dangerous. The classifications in these compilations are highly heterogeneous and in some cases wrong.

The sample of nearby AGNs emerging from the Sloan Digital Sky Survey (SDSS) (Kauffmann et al. 2003; Hao et al. 2005b; Kewley et al. 2006) far surpasses that of the Palomar survey in number but not in sensitivity. Moreover, because SDSS samples more distant galaxies, the $3^{\prime \prime}$-diameter fibers used in the survey subtend a physical scale of $\sim 5.5 \mathrm{kpc}$ at the typical redshift $z \approx 0.1,30$ times larger than in the Palomar survey. The SDSS spectra therefore include substantial contamination from off-nuclear emission, which dilutes and, in some cases, inevitably confuses the signal from the nucleus. Contamination by host galaxy emission has two consequences. First, only relatively bright nuclei have enough contrast to be detected. But second, contamination can introduce a more pernicious systematic effect that can be hard to quantify. Apart from normal H II regions, galactic disks are known to contain extended emission-line regions that exhibit low-ionization, LINER-like spectra. They can be confused with genuine nuclear LINERs. Examples include gas shocked by supernova remnants (e.g., Dopita \& Sutherland 1995), ejecta from starburst-driven winds (Armus, Heckman \& Miley 1990), and diffuse, warm ionized plasma (e.g., Collins \& Rand 2001). Massive, early-type galaxies, though generally lacking in ongoing star formation, also often possess X-ray emitting atmospheres that exhibit extended, low-ionization emission-line nebulae (e.g., Fabian et al. 1986; Heckman et al. 1989). These physical processes, while interesting in their own right, are not directly related, and thus irrelevant, to the AGN phenomenon. Thus, LINERs selected from samples of distant galaxies should be regarded with considerable caution. This comment applies also to LINERs selected from samples of IR-bright galaxies (e.g., Kim et al. 1995; Kewley et al. 2001; Corbett et al. 2003), which, in addition to being relatively distant and maximally confused with starburst processes, have the additional disadvantage of often being merging or interacting systems, wherein shocks undoubtedly generate extended LINER-like emission. I strongly recommend that researchers avoid IR-selected samples if they are interested in investigating LINERs as an accretion phenomenon. Many of the objects in the catalog of LINERs compiled by Carrillo et al. (1999), which has been the basis of several recent studies (Satyapal, Sambruna \& Dudik 2004; Dudik et al. 2005; González-Martín et al. 2006), suffer precisely from this complication and should be used judiciously.

\subsection{Detection Rates}

In qualitative agreement with early work, the Palomar survey shows that a substantial fraction (86\%) of all galaxies contain detectable emission-line nuclei (Ho, Filippenko \& Sargent 1997b). The detection rate is essentially $100 \%$ for all disk (S0 and spiral) galaxies, and $>50 \%$ for elliptical galaxies. One of the most surprising results is the large fraction of objects classified as AGNs or AGN candidates, as summarized in Figure 4. Summed over all Hubble types, $43 \%$ of all galaxies that fall in the survey limits can be considered "active." This percentage becomes even more remarkable for galaxies with an obvious bulge component, rising to $\sim 50 \%-70 \%$ for Hubble types E-Sb. By contrast, the detection rate of AGNs drops dramatically toward later Hubble types (Sc and later), which almost in- 

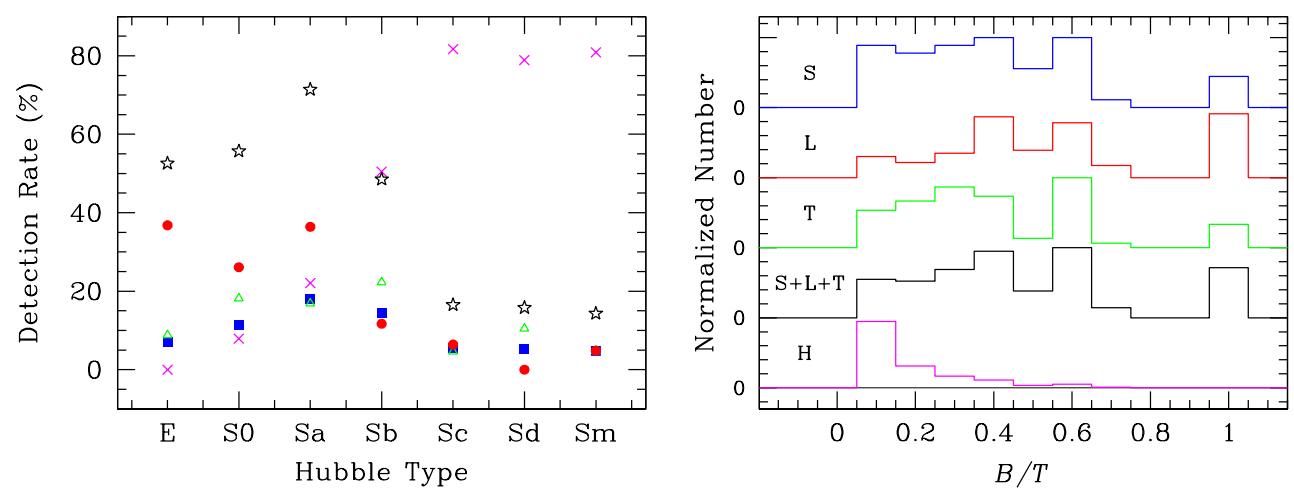

Figure 4: Left: Detection rate of emission-line nuclei as a function of Hubble type. The different classes of nuclei are marked as follows: Seyferts $=$ blue squares, LINERs $=$ red circles, transition objects $=$ green triangles, LINERs + transition objects + Seyferts $=$ black stars, H II nuclei = magenta crosses. Right: Distribution of bulge-to-total $(B / T)$ light ratios, derived from the morphological type and its statistical dependence on $B / T$. The histograms have been shifted vertically for clarity. (Adapted from Ho, Filippenko \& Sargent 1997a, 1997b.)

variably (80\%) host H II nuclei. This strong dependence of nuclear spectral class on Hubble type has been noticed in earlier studies (Heckman 1980a; Keel 1983b), and further confirmed in SDSS (Kauffmann et al. 2003; Miller et al. 2003). A qualitatively similar conclusion, cast in terms of host galaxy stellar mass rather than Hubble type, is reached by Gallo et al. (2008) and Decarli et al. (2007). Decarli et al. also claim that the occurrence of AGN activity in Virgo cluster spirals does not depend on morphological type, but it must be noted that the sources of spectroscopy and nuclear classification employed in that study are very heterogeneous.

Within the parent galaxy sample, $11 \%$ have Seyfert nuclei, at least doubling estimates based on older (Stauffer 1982b; Keel 1983b; Phillips, Charles \& Baldwin 1983) or shallower (Huchra \& Burg 1992; Maia, Machado \& Willmer 2003; Gronwall et al. 2004; Hao et al. 2005a) surveys. LINERs constitute the dominant population of AGNs. "Pure" LINERs are present in $\sim 20 \%$ of all galaxies, whereas transition objects, which by assumption also contain a LINER component, account for another $\sim 13 \%$. Thus, if all LINERs can be regarded as genuine AGNs (see $\S 6$ ), they truly are the most populous constituents, making up $1 / 3$ of all galaxies and $2 / 3$ of the AGN population (here taken to mean all objects classified as Seyferts, LINERs, and transition objects).

Within the magnitude range $14.5<r<17.7$ in SDSS, Kauffmann et al. (2003) report an overall AGN fraction (for narrow-line sources) of $\sim 40 \%$, of which $\sim 10 \%$ are Seyferts. The rest are LINERs and transition objects. Using a different method of starlight subtraction, Hao et al. (2005b) obtain very similar statistics for their sample of Seyfert galaxies. Roughly $30 \%$ of the galaxies on the red sequence in SDSS exhibit LINER-like emission (Yan et al. 2006). Although these detection rates broadly resemble those of the Palomar survey, one should recognize important differences between the two surveys. The Palomar objects extend much farther down the luminosity function than the SDSS. The emission- 
line detection limit of the Palomar survey, $\mathrm{EW}=0.25 \AA$, is roughly 10 times fainter than the cutoff chosen by Hao et al. (2005b). The faint end of the Palomar $\mathrm{H} \alpha$ luminosity function reaches $\sim 1 \times 10^{37} \mathrm{ergs} \mathrm{s}^{-1}$ (§ 5.9), again a factor of 10 lower than the SDSS counterpart. Given that LINERs selected from SDSS are highly susceptible to extranuclear contamination, as discussed earlier, it is in fact quite surprising - and perhaps fortuitous - that the detection rates of these objects agree so well between the two surveys.

\subsection{Broad Emission Lines}

Broad emission lines, a defining attribute of classical Seyferts and quasars, are also found in nuclei of much lower luminosities. The well-known case of the nucleus of M81 (Peimbert \& Torres-Peimbert 1981; Filippenko \& Sargent 1988), for example, has a broad (FWHM $\approx 3000 \mathrm{~km} \mathrm{~s}^{-1}$ ) $\mathrm{H} \alpha$ line with a luminosity of $2 \times 10^{39} \mathrm{ergs} \mathrm{s}^{-1}$ (Ho, Filippenko \& Sargent 1996), and many other less conspicuous cases have been discovered in the Palomar survey (Ho et al. 1997e). Searching for broad $\mathrm{H} \alpha$ emission in nearby nuclei is nontrivial, because it entails measurement of a (generally) weak, low-contrast, broad emission feature superposed on a complicated stellar background. Thus, the importance of careful starlight subtraction cannot be overemphasized. Moreover, even if this could be accomplished perfectly, one still has to contend with deblending the $\mathrm{H} \alpha+[\mathrm{N} \mathrm{II}]$ complex. The narrow lines in this complex are often heavily blended together, and rarely do the lines have simple profiles. The strategy adopted by Ho et al. (1997e) is to use the empirical line profile of the [S II] lines to model [N II] and the narrow component of $\mathrm{H} \alpha$. The case of NGC 3998 is shown in Figure 3.

Of the 221 emission-line nuclei in the Palomar survey classified as LINERs, transition objects, and Seyferts, 33 (15\%) definitely have broad $\mathrm{H} \alpha$ and an additional $16(7 \%)$ probably do. Questionable detections were found in another 8 objects (4\%). Thus, approximately $20 \%-25 \%$ of all nearby AGNs are type 1 sources. These numbers, of course, should be regarded as lower limits, since undoubtedly there must exist AGNs with even weaker broad-line emission that fall below the detection threshold. Although the numbers are meager, direct comparison with small-aperture Hubble Space Telescope (HST) spectra (e.g., Nicholson et al. 1998; Barth et al. 2001b; Shields et al. 2007) reveals that the Palomar statistics on broad $\mathrm{H} \alpha$ detections seem to be quite robust. The type 1.9 classification of almost every object with overlapping $H S T$ data turns out to survive. More surprising still, no new cases of broad $\mathrm{H} \alpha$ emission have turned up from HST observations. Given the difficulty of measuring the weak broad $\mathrm{H} \alpha$ feature on top of the dominant stellar continuum, it is likely that in general the line widths may have been systematically underestimated from the ground-based spectra. Circumstantial support for this conjecture comes from Zhang, DultzinHacyan \& Wang (2007), who find that Palomar LLAGNs tend to have smaller $\mathrm{BH}$ virial masses (estimated from the $\mathrm{H} \alpha$ linewidth and a BLR size-luminosity relation) than predicted from their bulge stellar velocity dispersion. They conclude that the BLR size in LLAGNs may be larger than normal given their luminosity, but an equally plausible explanation is that the $\mathrm{H} \alpha$ linewidths have been systematically underestimated.

It is illuminating to consider the incidence of broad $\mathrm{H} \alpha$ emission as a function of spectral class. Among objects formally classified as Seyferts, $\sim 40 \%$ are Seyfert 1 s. The implied ratio of Seyfert $1 \mathrm{~s}$ to Seyfert $2 \mathrm{~s}$ (1:1.6) has important consequences 
for some models concerning the evolution and small-scale geometry of AGNs (e.g., Osterbrock \& Shaw 1988). Within the Palomar sample, nearly $25 \%$ of the "pure" LINERs have detectable broad $\mathrm{H} \alpha$ emission. By direct analogy with the historical nomenclature established for Seyferts, LINERs can be divided into "type 1" and "type 2" sources according to the presence or absence of broad-line emission, respectively (Ho, Filippenko \& Sargent 1997a; Ho et al. 1997e). The detection rate of broad $\mathrm{H} \alpha$, however, drops drastically for transition objects. The cause for this dramatic change is unclear. In these objects the broad-line component may simply be too weak to be detected in the presence of substantial contamination from the H II region component, or it may be intrinsically absent ( $\S 5.5,6.5)$.

\subsection{Robustness and Completeness}

To gain confidence in the AGN statistics based on optical spectroscopy, one must have some handle on whether the existing AGN detections are trustworthy and whether there are many AGNs that have been missed. The robustness issue hinges on the question of whether the weak, nearby sources classified as AGNs are truly accretion-powered. As I argue in $\S 6$, this appears largely to be the case. The completeness issue can be examined in two regimes. Among galaxies with prominent bulges (Sbc and earlier), for which the spectroscopic AGN fractions are already very high $(\sim 50 \%-75 \%)$, there is not much room for a large fraction of missing AGNs, although it is almost certain that some have indeed eluded detection in the optical (e.g., Tzanavaris \& Georgantopoulos 2007). The same does not necessarily hold for galaxies of Hubble types Sc and later. While the majority of these systems are spectroscopically classified as H II nuclei, one must be wary that weak AGNs, if present, may be masked by brighter off-nuclear H II regions or H II regions projected along the line of sight. After all, some very late-type galaxies do host bona fide AGNs (see $\S 7$ ).

The AGN content of late-type galaxies can independently be assessed by using a diagnostic less prone to confusion by star-forming regions, namely by looking for compact, nuclear radio or X-ray cores. Ulvestad \& Ho (2002) performed a Very Large Array (VLA) survey for radio cores in a distance-limited sample of 40 Palomar Sc galaxies classified as hosting H II nuclei. To a sensitivity limit of $P_{\text {rad }} \approx 10^{18}-10^{20} \mathrm{~W} \mathrm{~Hz}^{-1}$ at $5 \mathrm{GHz}$, and a resolution of $\Delta \theta=1^{\prime \prime}$, they found that none of the galaxies contains a compact radio core. The VLA study of Filho, Barthel \& Ho (2000) also failed to detect radio cores in a more heterogeneous sample of $12 \mathrm{H}$ II nuclei.

Information on nuclear X-ray cores in late-type galaxies is much more limited because to date there has been no systematic investigation of these systems with Chandra. A few studies, however, have exploited the High Resolution Imager (HRI) on ROSAT to resolve the soft X-ray $(0.5-2 \mathrm{keV})$ emission in late-type galaxies (Colbert \& Mushotzky 1999; Lira, Lawrence \& Johnson 2000; Roberts \& Warwick 2000). Although the resolution of the HRI $\left(\sim 5^{\prime \prime}\right)$ is not ideal, it is nonetheless quite effective for identifying point sources given the relatively diffuse morphologies of late-type galaxies. Compact X-ray sources, often quite luminous $\left(z 10^{38} \mathrm{ergs} \mathrm{s}^{-1}\right)$, are frequently found, but generally they do not coincide with the galaxy nucleus; the nature of these off-nuclear "ultraluminous X-ray sources" is discussed by Fabbiano (2006).

To summarize: unless H II nuclei in late-type galaxies contain radio and X-ray cores far weaker than the current survey limits - a possibility worth exploring- 
they do not appear to conceal a significant population of undetected AGNs.

\section{HOST GALAXY PROPERTIES}

\subsection{Global Parameters}

The near dichotomy in the distribution of Hubble types for galaxies hosting active versus inactive nuclei (Figure 4) leads to the expectation that the two populations ought to have fairly distinctive global, and perhaps even nuclear, properties. Moreover, a detailed examination of the host galaxies of AGNs may shed light on the origin of their spectral diversity. These issues were examined by Ho, Filippenko \& Sargent (2003) using the database from the Palomar survey. The host galaxies of Seyferts, LINERs, and transition objects display a remarkable degree of homogeneity in their large-scale properties, after factoring out spurious differences arising from small mismatches in Hubble type distribution. The various nuclear types have slightly different Hubble distributions, which largely control many of the statistical properties of the host galaxies. Unless this effect is taken into account, one can arrive at erroneous conclusions about the intrinsic differences of the AGN populations. This is a crucial step, one that is often not appreciated. All three classes have essentially identical total luminosities $\left(\sim L^{*}\right)$, bulge luminosities, sizes, and neutral hydrogen content. Moreover, no obvious differences are found in terms of integrated optical colors or far-IR luminosities and colors, which implies very similar global stellar content and current star formation rates. No clear differences in environment can be seen either. The only exception is that, relative to LINERs, transition objects may show a mild enhancement in the level of global star formation, and they may be preferentially more inclined. This is consistent with the hypothesis that the transition class arises from spatial blending of emission from a LINER and H II regions. The velocity field of the ionized gas within the nuclear region, as measured by the width and asymmetry of the narrow emission lines, is crudely similar among the three AGN classes, an observation that argues against the proposition that fast shocks primarily drive the spectral variations $(\S 6.2)$.

\subsection{Nuclear Stellar Populations}

The uniformity in the global stellar populations among the three AGN classes extends to circumnuclear and even nuclear scales. The Palomar spectra cover a suite of stellar absorption-line indices and nuclear continuum colors, which collectively can be used to obtain crude constraints on the age and metallicity of the stars within the central $2^{\prime \prime}(\sim 200 \mathrm{pc})$. After isolating a subsample that mitigates the Hubble type dependence, Ho, Filippenko, \& Sargent (2003) find that Seyferts, LINERs, and transition objects have very similar stellar content. The same holds true when comparing type 1 and type 2 objects, both for LINERs and Seyferts. With a few notable exceptions such as NGC 404 and NGC 4569 (Maoz et al. 1998; Barth \& Shields 2000; Gabel \& Bruhweiler 2002) or NGC 4303 (Colina et al. 2002), the stellar population always appears evolved. Similar findings have been reported for smaller samples of LINERs, based on both optical (Boisson et al. 2000; Serote-Roos \& Gonçalves 2004; Zhang, Gu \& Ho 2008) and near-IR spectroscopy (Larkin et al. 1998; Bendo \& Joseph 2004). The optical regime is not well suited to detect very young, ionizing stars. However, the Palomar spectra 
do cover the broad He II $\lambda 4686$ emission bump, a feature indicative of Wolf-Rayet stars commonly seen in very young $(3-6 \mathrm{Myr})$ starbursts. Notwithstanding the difficulty of detecting this feature on top of a dominant old population, it is noteworthy that not a single case has been seen among the sample of over 200 Palomar LLAGNs. By contrast, the Wolf-Rayet bump has been found in a number of the H II nuclei (Sargent \& Filippenko 1991; Ho, Filippenko \& Sargent 1995), which, as a class compared to the LLAGNs, exhibit markedly younger stars, as evidenced by their blue continuum, strong $\mathrm{H} \beta$ and $\mathrm{H} \delta$ absorption, and weak metal lines (Ho, Filippenko \& Sargent 2003). The general dearth of young, massive stars in LLAGNs presents a serious challenge to proposals that seek to account for the excitation of their line emission in terms of starburst models.

Closer in, on scales $\lesssim 10$ pc accessible by HST, Sarzi et al. (2005) studied the nuclear stellar population for a distance-limited subsample of 18 Palomar LLAGNs. Their population synthesis analysis shows that the majority (80\%) of the objects have predominantly old $(z 5 \mathrm{Gyr})$, mildly reddened stars of near-solar metallicity, the only exceptions being 3 out of 6 transition objects and 1 out of 4 LINER $2 \mathrm{~s}$ that require a younger $(\$ 1 \mathrm{Gyr}$ ) component. In no case, however, is the younger component ever energetically dominant, falling far short of being able to account for the ionization budget for the central region.

The results of Ho, Filippenko \& Sargent (2003) have been disputed by Cid Fernandes et al. (2004), who obtained new ground-based spectra for a subset of the Palomar LINERs and transition objects. González Delgado et al. (2004), in a study similar to that of Sarzi et al. (2005), further analyzed STIS spectra of some of these. An important improvement of their ground-based data is that they extend down to $\sim 3500 \AA$, covering the $4000 \AA$ break and the higher-order Balmer lines, which are sensitive probes of intermediate-age $\left(\sim 10^{7}-10^{9} \mathrm{yr}\right)$ stars. While LINERs are predominantly old, roughly half of the transition nuclei show significant higher-order Balmer lines. Again, there are virtually no traces of Wolf-Rayet features. These authors propose that the ionization mechanism of transition sources must be somehow linked to the intermediate-age stellar population.

I disagree with their assessment. Figure 1 of Cid Fernandes et al. (2004) clearly shows that, as in the parent Palomar sample, the Hubble type distribution of the LINERs is skewed toward much earlier types than that of the transition objects. Any meaningful comparison of the stellar population, which strongly depends on Hubble type, must take this into account. The strategy employed by Ho, Filippenko \& Sargent (2003) is to restrict the two-sample comparisons to a relatively narrow range of Hubble types (Sab-Sbc). Within this domain, LINERs and transition objects (as well as Seyferts) have statistically indistinguishable stellar indices and continuum colors. Given the limited size of Cid Fernandes et al.'s sample, it is not possible to adopt the same strategy. To minimize the Hubble type bias, I examined the subsample of 30 spiral galaxies in their study. Out of 17 transition objects, $15(88 \%)$ contain intermediate-age stars according to their Table 3; but so do 10 out of the 13 (77\%) LINERs in this subgroup. This simple exercise underscores the importance of sample selection effects, and leaves me unconvinced that transition objects have a younger stellar population than LINERs. True, both classes evidently do contain detectable amounts of intermediate-age stars - a qualitatively different conclusion than was reached in the Palomar survey, whose spectral coverage was not well-suited to detect this population - but the fact remains that in a relative sense all three LLAGN classes 
in the Palomar survey have statistically similar populations. If the poststarburst component is responsible for the nebular emission in LLAGNs, we might expect the intensity of the two to be correlated, by analogy with what has been found for higher luminosity AGNs (Kauffmann et al. 2003). I searched for this effect in the final sample presented in González Delgado et al. (2004), but did not find any correlation. Clearly we wish to know what factors drive the spectral variations in LLAGNs; whatever they are $(\S 6)$, they are unlikely to be related to stellar population.

\subsection{Influence of Bars and Environment}

Numerical simulations (e.g., Heller \& Shlosman 1994; see review in Kormendy \& Kennicutt 2004) suggest that large-scale stellar bars can be highly effective in delivering gas to the central few hundred parsecs of a spiral galaxy, thereby potentially leading to rapid star formation. Further instabilities result in additional inflow to smaller scales, which may lead to increased $\mathrm{BH}$ fueling and hence elevated nonstellar activity in barred galaxies compared to unbarred galaxies. As discussed in $\S 3.1$, the Palomar sample is ideally suited for statistical comparisons of this nature, which depend delicately on issues of sample completeness and the choice of control sample. Ho, Filippenko \& Sargent (1997d) find that while the presence of a bar indeed does enhance both the probability and rate of star formation in galaxy nuclei, it appears to have no impact on either the frequency or strength of AGN activity. Bearing in mind the substantial uncertainties associated with sample selection, as well as the method and wavelength used to identify bars (Laurikainen, Salo \& Buta 2004), other studies broadly come to a similar conclusion (see review by Combes 2003), although Maia, Machado \& Willmer (2003) claim, on the basis of a significantly larger and somewhat more luminous sample drawn from the Southern Sky Redshift Survey, that Seyfert galaxies are preferentially more barred.

In the same vein, dynamical interactions with neighboring companions should lead to gas dissipation, enhanced nuclear star formation, and perhaps central fueling (e.g., Hernquist 1989). Schmitt (2001) and Ho, Filippenko \& Sargent (2003) studied this issue using the Palomar data, parameterizing the nearby environment of each object by its local galaxy density and the distance to its nearest sizable neighbor. After accounting for the well-known morphology-density relation, it was found that the local environment, like bars, has little impact on AGNs, at least in the low-luminosity regime sampled locally. These findings broadly agree with the results from SDSS (Miller et al. 2003; Li et al. 2008). Kauffmann et al. (2004), Wake et al. (2004), and Constantin \& Vogeley (2006), in fact, report a drop in the fraction of high-luminosity AGNs for dense environments.

\section{NUCLEAR PROPERTIES}

\subsection{Ionizing Continuum Radiation}

AGNs, at least when unobscured, reveal themselves as pointlike nuclear sources with power-law spectra at optical and UV wavelengths, typically described by a continuum flux density $f_{\nu} \propto \nu^{\alpha}$, with $\alpha \approx-0.5$ (e.g., Vanden Berk et al. 2001). In unbeamed sources, this featureless continuum traces the low-frequency tail of the "big blue bump" (Shields 1978; Malkan \& Sargent 1982), which sup- 
plies the bulk of the ionizing photons. This feature is extremely difficult to detect in LLAGNs, both because the big blue bump is weak or absent $(\S 5.8)$ and because the sources are exceedingly faint. The optical nuclei of LINERs can have $M_{B} \gtrsim-10 \mathrm{mag}$ (Ho 2004b), at least $10^{4}$ times fainter than their (usually bulge-dominated) hosts $\left(M_{B} \simeq M^{*} \approx-20 \mathrm{mag}\right)$. To overcome this contrast problem, searches for nuclear point sources in the optical and near-IR have relied on HST images (e.g., Chiaberge, Capetti \& Celotti 1999; Quillen et al. 2001; Verdoes Kleijn et al. 2002; Chiaberge, Capetti \& Macchetto 2005; Balmaverde \& Capetti 2006; González-Martín et al. 2006). But resolution alone is not enough. Given the extreme faintness of the nucleus, the intrinsic cuspiness of the underlying bulge profile, complexities of the point-spread function, and the often irregular background marred by circumnuclear dust features, one must pay very close attention to how the measurements are made. Simple aperture photometry or searching for central excess emission can yield very misleading results. The most robust technique to extract faint nuclei in the presence of these complications employs two-dimensional, multi-component fitting (Ho \& Peng 2001; Ravindranath et al. 2001; Peng et al. 2002). Using this method, nuclear sources with optical magnitudes as faint as $\sim 20$ have been measured, with limits down to $\sim 22-23$ mag possible for nearby galaxies. Due to the computational requirements of twodimensional fitting, however, not many LLAGNs have yet been analyzed in this manner, and fewer still have enough photometric points to define even a crude spectral slope.

In a few cases, the optical featureless continuum has been detected spectroscopically. From the ground, this was only possible for a couple of the brightest sources. The stellar features of NGC 7213 (Halpern \& Filippenko 1984) and Pictor A (Carswell et al. 1984; Filippenko 1985) show dilution by a featureless continuum, which can be described approximately by a power law with a spectral index of $\alpha \approx-1.5$. The nuclear continuum is much more readily seen in small-aperture spectra that help to reject the bulge starlight. HST spectra have isolated the optical continuum in several LINERs (Ho, Filippenko \& Sargent 1996; Nicholson et al. 1998; Ho et al. 2000; Shields et al. 2000; Barth et al. 2001a; Sabra et al. 2003), although in most objects it remains too faint to be detected spectroscopically (Sarzi et al. 2005). In all well-studied cases, the optical continuum is quite steep, with $\alpha \approx-1$ to -2 . This range in spectral slopes is consistent with the broad-band optical (Verdoes Kleijn et al. 2002) and optical-UV colors (Chiaberge et al. 2002) of the cores frequently detected in the LINER nuclei of FR I radio galaxies.

The predominantly old population of present-day bulges ensures that the stellar contamination largely disappears in the UV, especially at high resolution. A number of attempts have been made to detect UV emission in LINERs using $I U E$, but most of these efforts yielded ambiguous results (see review in Filippenko 1996), and real progress had to await the HST. Two dedicated HST UV $(\sim 2300$ $\AA$ ) imaging studies have been completed. Using the pre-COSTAR FOC, Maoz et al. (1996) surveyed a complete sample of 110 large, nearby galaxies, and among the subset with spectral classifications from Palomar, Maoz et al. (1995) discovered that $\sim 25 \%$ of the LINERs show an unresolved UV core. Barth et al. (1998) found similar statistics in a more targeted WFPC2 study. They also made the suggestion, later confirmed by Pogge et al. (2000), that dust obscuration is probably the main culprit for the nondetection of UV emission in the majority of LINERs. The implication is that UV emission is significantly 
TABLE 1

MEDIAN STATISTICAL PROPERTIES OF LLAGNS

\begin{tabular}{|c|c|c|c|c|c|c|c|c|c|c|}
\hline $\begin{array}{c}\text { Spectral } \\
\text { Class } \\
(1)\end{array}$ & $\begin{array}{c}L_{\mathrm{H} \alpha} \\
\left(\mathrm{ergs} \mathrm{s}^{-1}\right) \\
(2)\end{array}$ & $\begin{array}{c}L_{\mathrm{X}} \\
\left(\operatorname{ergs~s}^{-1}\right) \\
(3)\end{array}$ & $\begin{array}{c}P_{\mathrm{rad}} \\
\left(\mathrm{W} \mathrm{Hz}^{-1}\right) \\
(4)\end{array}$ & $\begin{array}{c}L_{\mathrm{bol}} / L_{\mathrm{Edd}} \\
(5)\end{array}$ & $\begin{array}{c}L_{\mathrm{X}} / L_{\mathrm{H} \alpha} \\
(6)\end{array}$ & $\begin{array}{c}\log R_{o} \\
(7)\end{array}$ & $\begin{array}{c}\log R_{\mathrm{X}} \\
\quad(8)\end{array}$ & $\begin{array}{c}f_{b} \\
(\%) \\
(9)\end{array}$ & $\begin{array}{c}f_{r} \\
(\%) \\
(10)\end{array}$ & $\begin{array}{r}f_{x} \\
(\%) \\
(11)\end{array}$ \\
\hline S1 & $1.9 \times 10^{40}$ & $1.4 \times 10^{41}$ & $8.5 \times 10^{20}$ & $1.1 \times 10^{-3}$ & 7.3 & 0.7 & -3.8 & 52 & 72 & 100 \\
\hline $\mathrm{S} 2$ & $1.7 \times 10^{39}$ & $1.3 \times 10^{39}$ & $8.5 \times 10^{19}$ & $5.9 \times 10^{-6}$ & 0.75 & 0.4 & -3.3 & $\ldots$ & 30 & 86 \\
\hline L1 & $3.7 \times 10^{39}$ & $8.8 \times 10^{39}$ & $2.6 \times 10^{20}$ & $1.0 \times 10^{-5}$ & 4.6 & 1.3 & -2.9 & 23 & 63 & 95 \\
\hline L2 & $0.5 \times 10^{39}$ & $1.2 \times 10^{39}$ & $4.7 \times 10^{19}$ & $4.8 \times 10^{-6}$ & 1.6 & 0.8 & -2.9 & $\ldots$ & 38 & 74 \\
\hline $\mathrm{T}$ & $0.7 \times 10^{39}$ & $0.5 \times 10^{39}$ & $2.4 \times 10^{19}$ & $1.2 \times 10^{-6}$ & 0.41 & 0.4 & -2.8 & 3 & 16 & 74 \\
\hline
\end{tabular}

NOTE.- Column (1) Spectral class. (2) Extinction-corrected luminosity of the H $\alpha$ line; for type 1 sources, it represents both the narrow and broad components. (3) X-ray luminosity in the $2-10 \mathrm{keV}$ band. (4) Radio power at $5 \mathrm{GHz}$. (5) Bolometric Eddington ratio, with $L_{\mathrm{bol}}$ estimated from $L_{\mathrm{X}}$ and $L_{\mathrm{Edd}}$ derived from $\mathrm{BH}$ masses estimated using the $M_{\mathrm{BH}}-\sigma$ relation of Tremaine et al. 2002. (6) Ratio of X-ray to $\mathrm{H} \alpha$ luminosity. (7) Optical radio-loudness parameter. (8) X-ray radio-loudness parameter. (9) Detection fraction of broad $\mathrm{H} \alpha$ emission. (10) Detection fraction of radio cores at $15 \mathrm{GHz}$. (11) Detection fraction of X-ray cores.

more common in LINERs than indicated by the detection rates. In some type 2 objects (e.g., NGC 4569 and NGC 6500), the UV emission is spatially extended and presumably not related to the nuclear source. Second-epoch UV observations with the ACS/HRC revealed that nearly all of the UV-bright sources exhibit longterm variability (Maoz et al. 2005), an important result that helps assuage fears that the UV emission might arise mainly from young stars (Maoz et al. 1998). Importantly, both type 1 and type 2 LINERs vary. UV variability has also been discovered serendipitously in a few other sources (Renzini et al. 1995; O'Connell et al. 2005).

\subsection{Radio Cores}

AGNs, no matter how weak, are almost never silent in the radio. Barring chance superposition with a supernova remnant, the presence of a compact radio core is therefore a good AGN indicator. Because of the expected faintness of the nuclei, however, any search for core emission must be conducted at high sensitivity, and arcsecond-scale angular resolution or better is generally needed to isolate the nucleus from the surrounding host, which emits copious diffuse synchrotron radiation. In practice, this requires an interferometer such as the VLA.

The prevalence of weak AGNs in nearby early-type galaxies has been established from the VLA radio continuum studies of Sadler, Jenkins \& Kotanyi (1989) and Wrobel \& Heeschen (1991), whose $5 \mathrm{GHz}$ surveys with $\Delta \theta \approx 5^{\prime \prime}$ report a high incidence $(\sim 30 \%-40 \%)$ of radio cores in complete, optical flux-limited samples of elliptical and S0 galaxies. Interestingly, the radio detection rate is similar to the detection rate of optical emission lines (Figure 4), and the optical counterparts of the radio cores are mostly classified as LINERs (Phillips et al. 1986; Ho 1999a). Conversely, Heckman (1980b) showed that LINERs tend to be associated with compact radio sources. The radio powers are quite modest, generally in the range of $10^{19}-10^{21} \mathrm{~W} \mathrm{~Hz}^{-1}$ at $5 \mathrm{GHz}$. When available, the spectral indices tend to be relatively flat (e.g., Wrobel 1991; Slee et al. 1994). With the exception of a handful of well-known radio galaxies with extended jets (Wrobel 1991), most of the radio emission is centrally concentrated.

No comparable radio survey has been done for spiral galaxies. Over the last few years, however, a number of studies, mostly using the VLA, have systematically targeted sizable subsets of the Palomar galaxies, to the point that by 
now effectively the entire Palomar AGN sample has been surveyed at arcsecond $\left(\Delta \theta \approx 0{ }^{\prime \prime} 15-2{ }^{\prime \prime} 5\right)$ resolution (Filho, Barthel \& Ho 2000, 2002a, 2006; Nagar et al. 2000, 2002; Ho \& Ulvestad 2001; Filho et al. 2004; Nagar, Falcke \& Wilson 2005; Krips et al. 2007). Because the sensitivity, resolution, and observing frequency varied from study to study, each concentrating on different subclasses of objects, it is nontrivial to combine the literature results. The only survey that samples a significant fraction of the three LLAGN classes at a uniform sensitivity and resolution is that by Nagar et al. (2000, 2002; Nagar, Falcke \& Wilson 2005), which was conducted at $15 \mathrm{GHz}$ and $\Delta \theta=0$ " 15 . The main drawback is that the sensitivity of this survey ( $\mathrm{rms} \approx 0.2 \mathrm{mJy}$ ) is rather modest, and mJy-level sources can be missed if they possess relatively steep spectra. Despite these limitations, Nagar et al. detected a compact core, to a high level of completeness, in $44 \%$ of the LINERs. Importantly, to the same level of completeness, the Seyferts exhibit a very similar detection rate $(47 \%)$. LINER 2 s have a lower detection rate than LINER 1s (38\% versus $63 \%$; see Table 1$)$, but the same pattern is reflected almost exactly within the Seyfert population (detection rate $30 \%$ for type 2 s versus $72 \%$ for type $1 \mathrm{~s})$. Transition objects, on the other hand, clearly differ, showing a markedly lower detection rate of only $16 \%$, consistent with the $8.4 \mathrm{GHz}$ survey of Filho, Barthel \& Ho (2000, 2002a, 2006), where the detection rate is $\sim 25 \%$. The statistical differences in the Hubble type distributions of the three AGN classes (Ho, Filippenko \& Sargent 2003) slightly complicate the interpretation of these results. To the extent that radio power shows a mild dependence on bulge strength or BH mass (Nagar, Falcke \& Wilson 2005; see Ho 2002a), the detection rates, strictly speaking, should be renormalized to account for the differences in morphological types among the three classes. This effect, however, will not qualitatively change the central conclusion: if a compact radio core guarantees AGN pedigree, then LINERs, of either type 1 or type 2, are just as AGN-like as Seyferts, whereas a significant fraction of transition objects (roughly half) may be unrelated to AGNs.

The detection rates from the Nagar et al. survey can be viewed as firm lower limits. At $\Delta \theta=1^{\prime \prime}$ and $\mathrm{rms}=0.04 \mathrm{mJy}$ at 1.4 and $5 \mathrm{GHz}$, for example, the detection rate for the Palomar Seyferts rises to $75 \%$ (Ho \& Ulvestad 2001). Although no lower frequency survey of LINERs has been completed so far (apart from the lower resolution studies of Sadler, Jenkins, \& Kotanyi 1989 and Wrobel \& Heeschen 1991 confined to early-type galaxies), the preliminary study by Van Dyk \& Ho (1998) of 29 LINERs at 5 and $3.6 \mathrm{GHz}(\Delta \theta=0 . / 5$; rms $=0.05-0.1$ mJy) yielded a detection rate of over $80 \%$, again suggesting that LINERs and Seyferts have a comparably high incidence of radio cores.

Importantly, a sizable, flux-limit subset of the $15 \mathrm{GHz}$ detections has been reobserved with the Very Long Baseline Array at $5 \mathrm{GHz}$, and essentially all of them have been detected at milliarcsecond resolution (Nagar, Falcke \& Wilson 2005). The high brightness temperatures $\left(\gtrsim 10^{6}-10^{11} \mathrm{~K}\right)$ leaves no doubt that the radio cores are nonthermal and genuinely associated with AGN activity.

Where multifrequency data exist, their spectra tend to be flat or even mildly inverted $(\alpha \approx-0.2$ to +0.2 ; Ho et al. 1999b; Falcke et al. 2000; Nagar et al. 2000; Nagar, Wilson \& Falcke 2001; Ulvestad \& Ho 2001b; Anderson, Ulvestad \& Ho 2004; Doi et al. 2005; Krips et al. 2007), seemingly more optically thick than Seyferts (median $\alpha=-0.4$; Ulvestad \& Ho 2001a), and variability on timescales of months is common (Nagar et al. 2002; Anderson \& Ulvestad 2005). Both of these characteristics suggest that the radio emission in LINERs is mainly confined 
to a compact core or base of a jet. Seyfert galaxies contain radio cores as well, but they are often accompanied by linear, jetlike features resolved on arcsecond scales (e.g., Ulvestad \& Wilson 1989; Kukula et al. 1995; Ho \& Ulvestad 2001; Gallimore et al. 2006). This extended component appears to be less prevalent in LINERs, although a definitive comparison must await a survey matched in resolution, sensitivity, and wavelength with that performed for the Seyferts (Ho \& Ulvestad 2001). Higher resolution images on milliarcsecond scales do resolve elongated structures akin to subparsec-scale jets, but most of the power is concentrated in a compact, high-brightness temperature core (Bietenholz, Bartel \& Rupen 2000; Falcke et al. 2000; Ulvestad \& Ho 2001b; Filho, Barthel \& Ho 2002b; Anderson, Ulvestad \& Ho 2004; Filho et al. 2004; Krips et al. 2007). The comprehensive summary presented in Nagar, Falcke \& Wilson (2005) indicates that the incidence of milliarcsecond-scale radio cores is similar for LINERs and Seyferts, but that subparsec-scale jets occur more frequently in LINERs.

\section{$5.3 \quad X$-ray Cores}

X-ray observations provide another very effective tool to isolate LLAGNs and to diagnose their physical properties. Ultra-faint LLAGNs can be identified where none was previously known in the optical (e.g., Loewenstein et al. 2001; Ho, Terashima \& Ulvestad 2003; Fabbiano et al. 2004; Pellegrini et al. 2007; Wrobel, Terashima \& Ho 2008). Here, too, sensitivity and resolution are critical, as the central regions of galaxies contain a plethora of discrete nonnuclear sources, often suffused with a diffuse thermal plasma. Chandra, whose ACIS camera delivers $\sim 0$. 5 images, is the instrument of choice, although in some instances even data at $\sim 5^{\prime \prime}$ resolution (e.g., ROSAT HRI) can still provide meaningful constraints, especially if accompanied by spectral information (e.g., XMM-Newton).

As in the radio, no truly unbiased high-resolution X-ray survey has yet been performed of an optical flux-limited sample of nearby galaxies. The closest attempt was made by Roberts \& Warwick (2000), who searched for X-ray nuclear sources in 83 Palomar galaxies ( $\sim 20 \%$ of the total sample) having archival ROSAT HRI data. This subset is probably not unbiased, but it does encapsulate all the nuclear spectral classes in the Palomar survey. In total, X-ray cores were detected in $54 \%$ of the sample, with Seyferts and LINERs (including transition objects) both showing a higher detection rate $(\sim 70 \%)$ than absorption $(30 \%)$ or H II nuclei (40\%). The high detection rate among the optically classified LLAGNs agrees well with other ROSAT studies of Palomar sources (Koratkar et al. 1995; Komossa, Böhringer \& Huchra 1999; Halderson et al. 2001; Roberts, Schurch \& Warwick 2001), but the nonnegligible detection rate among the inactive members suggests that a significant fraction of the "core" flux may be nonnuclear emission [X-ray binaries (XRBs) and diffuse gas] insufficiently resolved by ROSAT.

Observations with Chandra (e.g., Ho et al. 2001; Eracleous et al. 2002) confirm the suspicion that earlier X-ray studies may have suffered from confusion with extranuclear sources (Figure 5). Importantly, the sharp resolution and low background noise of ACIS enable faint point sources to be detected with brief (few ks) exposures. This makes feasible, for the first time, X-ray surveys of large samples of galaxies selected at non-X-ray wavelengths. In a snapshot survey of a distance-limited sample of Palomar LLAGNs, Ho et al. (2001) find that $\sim 75 \%$ of LINERs, both type 1 and type 2, contain X-ray cores, some as faint as $\sim 10^{38}$ ergs $\mathrm{s}^{-1}$ in the $2-10 \mathrm{keV}$ band. Terashima \& Wilson (2003b) report an even 

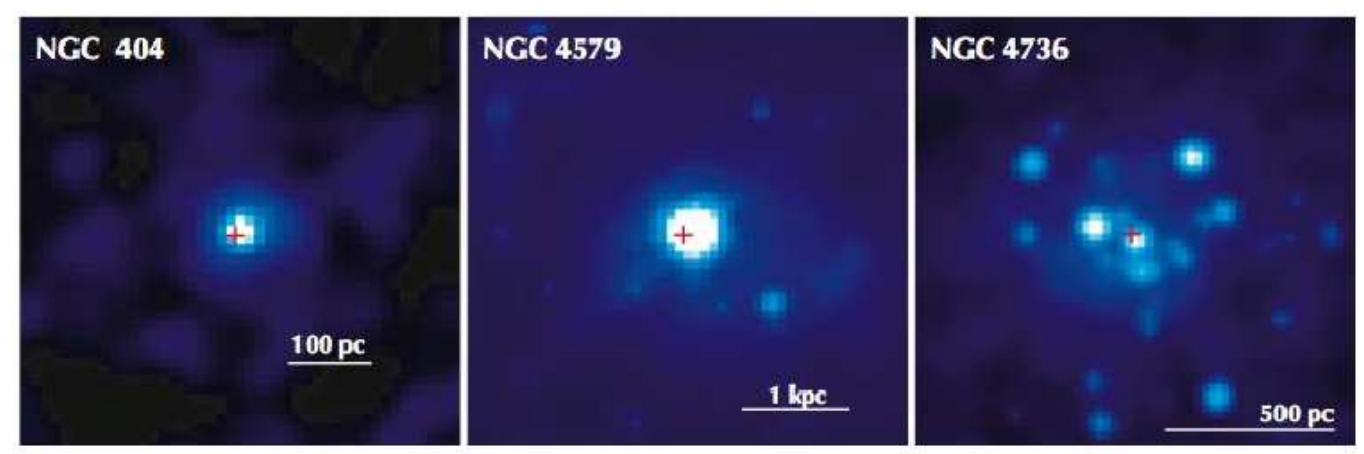

Figure 5: Chandra/ACIS images of three LLAGNs, illustrating the diversity and complexity of the X-ray morphologies of their circumnuclear regions. The cross marks the near-IR position of the nucleus. (Courtesy of H.M.L.G. Flohic and M. Eracleous.)

higher detection rate (100\%) for a sample of LINERs chosen for having a flatspectrum radio core. To date, roughly $50 \%$ of the entire Palomar sample, among them $40 \%$ of the AGNs, have been observed by Chandra. This rich archival resource has been the basis of a number of recent investigations focused on quantifying the AGN content of LINERs, chief among them Satyapal, Sambruna \& Dudik (2004), Dudik et al. (2005), Pellegrini (2005), Satyapal et al. (2005), Flohic et al. (2006), and González-Martín et al. (2006). A common conclusion that can be distilled from these studies is that the incidence of X-ray cores among LINERs is quite high, ranging from $\sim 50 \%$ to $70 \%$, down to luminosity limits of $\sim 10^{38} \mathrm{ergs} \mathrm{s}^{-1}$. The incidence of X-ray cores in LINERs is somewhat lower than, but still compares favorably to, that found in Palomar Seyferts $(\sim 90 \%)$, the vast majority of which now have suitable X-ray observations, as summarized in Cappi et al. (2006) and Panessa et al. (2006). While the impact of selection biases cannot be assessed easily, they are probably not very severe because most of the observations were not originally intended to study LINERs, nor were they targeting famous X-ray sources.

It is of interest to ask whether the incidence of X-ray cores in LINERs depends on the presence of broad $\mathrm{H} \alpha$ emission. The moderate-resolution ROSAT/HRI studies of Roberts \& Warwick (2000) and Halderson et al. (2001) showed roughly comparable detection rates for type 1 and type 2 LINERs, suggesting that the two classes are intrinsically similar and that obscuration plays a minor role in differentiating them. On the other hand, detailed X-ray spectral analysis has raised the suspicion that LINER 2s may be a highly heterogeneous class, with the bulk of the X-ray emission possibly arising from stellar processes. An important caveat is that these studies were based on large-beam observations, mostly using $A S C A$ (Terashima, Ho \& Ptak 2000; Terashima et al. 2000a, 2002; Roberts, Schurch \& Warwick 2001) and the rest using BeppoSAX (Georgantopoulos et al. 2002; Pellegrini et al. 2002). A clearer, more consistent picture emerges from the recent Chandra work cited above. Although the individual samples remain small, most Chandra surveys detect LINER 2 s with roughly similar frequency as LINER 1s, $\sim 50 \%-60 \%$. To gain a more comprehensive census, I have assembled Chandra measurements for all Palomar LINERs from the literature, along with unpublished material for a significant number of additional objects in public archives, 
which were analyzed following Ho et al. (2001). Although clearly heterogeneous and incomplete, the final collection of 64 LINERs (20 type 1, 44 type 2) does constitute $70 \%$ of the entire Palomar sample. The detection rate among all LINERs is $86 \%$, broken down into $95 \%$ for LINER 1s and $74 \%$ for LINER 2s. For completeness, note that a similar exercise for 36 transition objects $(55 \%$ of the parent sample) yields a detection rate of $74 \%$, identical to that of LINER $2 \mathrm{~s}$ and only marginally lower than that of Seyfert 2s (86\%; Table 1).

The X-ray spectral properties of LLAGNs, particularly LINERs, have most thoroughly been investigated using $A S C A$ (Yaqoob et al. 1995; Ishisaki et al. 1996; Iyomoto et al. 1996, 1997, 1998a, 1998b; Ptak et al. 1996, 1999; Terashima et al. 1998a, 1998b, 2000a, 2000b, 2002; Ho et al. 1999a; Terashima, Ho \& Ptak 2000; Roberts, Schurch \& Warwick 2001), with important contributions from BeppoSAX (Pellegrini et al. 2000a, 2000b, 2002; Iyomoto et al. 2001; Georgantopoulos et al. 2002; Ptak et al. 2004). A seminal study on M81 was done using BBXRT (Petre et al. 1993). Although the nuclear component was not spatially isolated because of the poor angular resolution of these telescopes, they had sufficient effective area to yield good photon statistics over the energy range $\sim 0.5-10 \mathrm{keV}$ to spectrally isolate the hard, power-law AGN signal. The most salient properties are the following. (1) Over the region $\sim 0.5-10 \mathrm{keV}$, the continuum can be fit with a power law with an energy index of $\alpha \approx-0.4$ to -1.2. Although this range overlaps with that seen in more luminous sources, the typical value of $\sim-0.8$ in LLAGNs may be marginally flatter than in Seyfert $1 \mathrm{~s}$ $(\langle\alpha\rangle=-0.87 \pm 0.22 ;$ Nandra et al. 1997b) or radio-quiet quasars $(\langle\alpha\rangle=-0.93 \pm$ 0.22 ; Reeves \& Turner 2000), perhaps being more in line with radio-loud quasars $(\langle\alpha\rangle=-0.6 \pm 0.16$; Reeves \& Turner 2000). (2) With a few notable exceptions (e.g., M51: Fukazawa et al. 2001, Terashima \& Wilson 2003a; NGC 1052: Weaver et al. 1999, Guainazzi et al. 2000; NGC 4258: Makishima et al. 1994, Fiore et al. 2001; NGC 4261: Matsumoto et al. 2001), the power-law component shows very little intrinsic absorption. This trend conflicts with the tendency for the degree of obscuration to increase with decreasing luminosity (e.g., Lawrence \& Elvis 1982). (3) Signatures of X-ray reprocessing by material from an optically thick accretion disk, in the form of $\mathrm{Fe} \mathrm{K} \alpha$ emission or Compton reflection (Lightman \& White 1988; George \& Fabian 1991), are weak or absent; the weakness of the Fe $\mathrm{K} \alpha$ line in LLAGNs runs counter to the inverse correlation between iron line strength and luminosity observed in higher luminosity AGNs (Nandra et al. 1997b). (4) In the few cases where Fe K $\alpha$ emission has been detected, it is always narrow. (5) Apart from the hard power law, most objects require an extra soft component at energies $\lesssim 2 \mathrm{keV}$ that can be fit by a thermal plasma model with a temperature of $k T \approx 0.4-0.8 \mathrm{keV}$ and near-solar abundances. (6) Contrary to the trend established for luminous sources (Nandra et al. 1997a), short-term, large-amplitude X-ray variability is rare in LLAGNs (Ptak et al. 1998).

More recent observations with Chandra and XMM-Newton have refined, but not qualitatively altered, the above results. Where detailed spectral analysis is possible (e.g., Böhringer et al. 2001; Kim \& Fabbiano 2003; Pellegrini et al. 2003a; Terashima \& Wilson 2003b; Filho et al. 2004; Page et al. 2004; Starling et al. 2005; Flohic et al. 2006; González-Martín et al. 2006; Soria et al. 2006), the hard power-law component (except in objects previously known to be heavily absorbed) continues to be relatively unabsorbed, even among many type 2 sources, and to show little signs of reflection. No convincing case of a relativistic Fe $\mathrm{K} \alpha$ line has yet surfaced in an LLAGN. The marginally broad iron lines 
discovered with $A S C A$ in M81 (Ishisaki et al. 1996) and NGC 4579 (Terashima et al. 1998a) has now been resolved into multiple components (Dewangan et al. 2004; Page et al. 2004; Young et al. 2007), none of which can be associated with a canonical disk. At the same time, the equivalent width limits for even the narrow component have become impressively low (e.g., Ptak et al. 2004). Interestingly, a soft thermal component is still required in many objects (§ 5.4), but there is no evidence for blackbody-like soft excess emission commonly seen in Seyferts and quasars (e.g., Turner \& Pounds 1989; Inoue, Terashima \& Ho 2007).

\subsection{Circumnuclear Thermal Plasma}

Early X-ray observations of LLAGNs using $A S C A$ have consistently revealed the presence of a diffuse, thermal component, typically with a temperature of $k T \approx 0.5 \mathrm{keV}$ (Ptak et al. 1999; Terashima et al. 2002). The uniform analysis of ROSAT data by Halderson et al. (2001) concluded that $\sim 80 \%$ of the Palomar sources contain an extended component. However, without better resolution, it was impossible to know the extent of confusion with point sources, how much of the gas is truly associated with the nuclear region of the galaxy, or the density and temperature profile of the gas.

Our view of the diffuse component in the nuclear region has been dramatically sharpened with Chandra and XMM-Newton. Not only has the near ubiquity of diffuse gas been confirmed in many nearby galaxies (Ho et al. 2001; Eracleous et al. 2002; Terashima \& Wilson 2003b; Pellegrini 2005; Rinn, Sambruna \& Gliozzi 2005; Cappi et al. 2006; González-Martín et al. 2006; Soria et al. 2006), including our own (Muno et al. 2004) and our close neighbor M31 (Garcia et al. 2005), but quantitative, statistical properties of the gas are now becoming available. In the comprehensive investigation of 19 LINERs by Flohic et al. (2006), the diffuse emission, detected in $70 \%$ of the sample, is concentrated within the central few hundred pc. With an average $0.5-2 \mathrm{keV}$ luminosity of $\sim 10^{38} \mathrm{ergs} \mathrm{s}^{-1}$, it accounts for more than half of the total central luminosity in most cases. The average spectrum is similar to that seen in normal galaxies: it can be described by a thermal plasma with $k T=0.5 \mathrm{keV}$ plus a power-law component with $\alpha=-0.3$ to -0.5 . I will return to the nature of the hard component in $\S 6.5$. What is the origin of the thermal plasma? Given what we know about the stellar populations ( $§ 4.2$ ), a starburst origin, as suggested by González-Martín et al. (2006), seems improbable. In normal elliptical galaxies, the X-ray-emitting gas represents the repository of thermalized stellar ejecta generated from mass loss from evolved stars and Type Ia supernovae (e.g., Awaki et al. 1994). There is no reason not to adopt the same picture to explain the hot plasma in LINERs and other LLAGNs. High-resolution X-ray spectroscopy of the highly ionized gas around the nucleus of M81 (Page et al. 2003; Young et al. 2007) and NGC 7213 (Starling et al. 2005) reveals that the plasma is collisionally ionized. Starling et al. note that this may be a property unique to LINERs, as thermal gas in luminous Seyferts is usually photoionized rather than collisionally ionized (e.g., Kinkhabwala et al. 2002).

\subsection{Broad-line Region}

Luminous, unobscured AGNs distinguish themselves unambiguously by their characteristic broad permitted lines. The detection of broad $\mathrm{H} \alpha$ emission in 


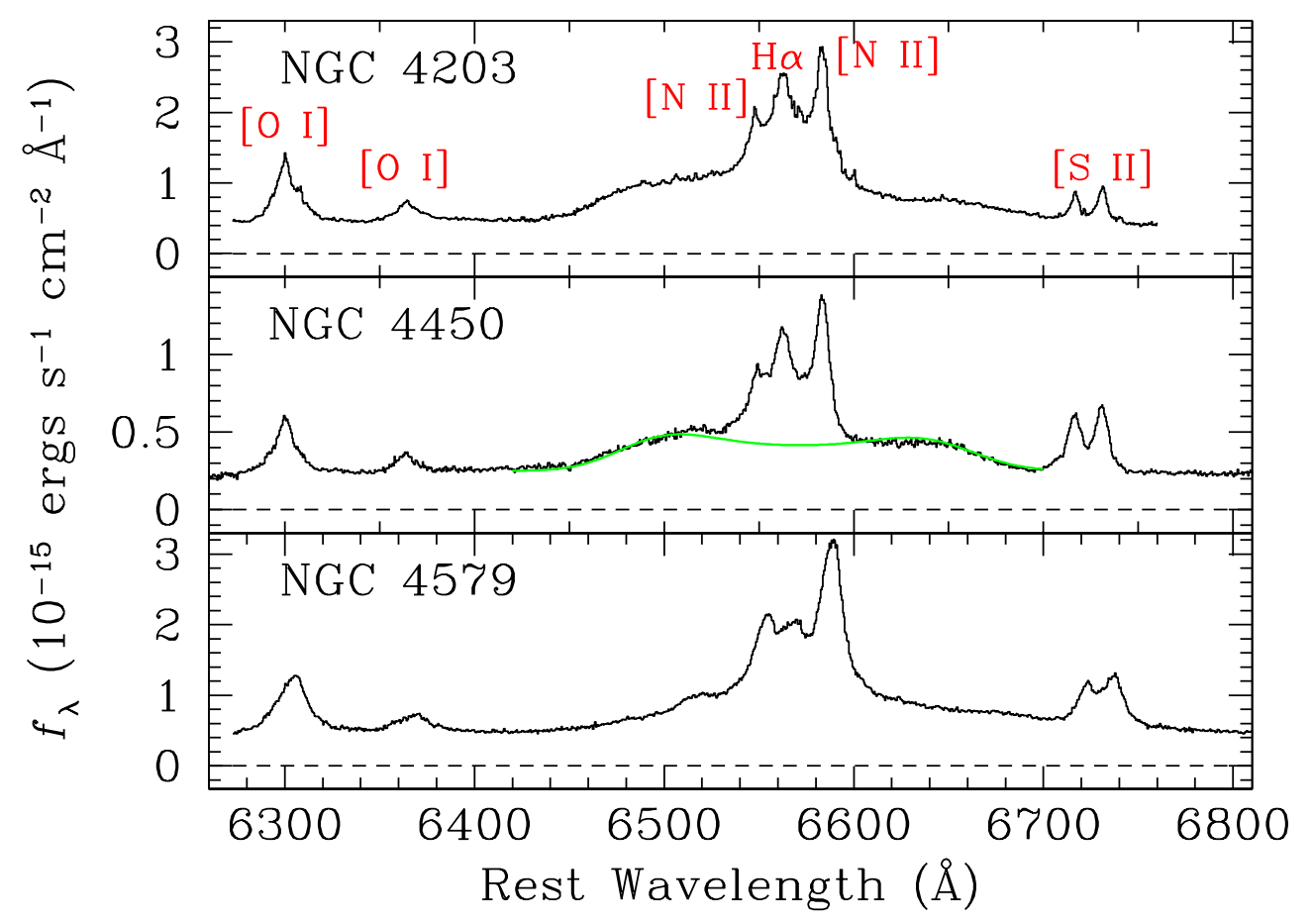

Figure 6: LINERs with broad, double-peaked $\mathrm{H} \alpha$ emission discovered with $H S T$. A model fit for the disk profile in NGC 4450 is shown for illustration (green curve). (Adapted from Ho et al. 2000, Shields et al. 2000, and Barth et al. 2001a.)

$\sim 25 \%$ of LINERs (Ho et al. 1997e) thus constitutes strong evidence in favor of the AGN interpretation of these sources. LINERs, like Seyferts, come in two flavors - some have a visible BLR (type 1), and others do not (type 2). The broad component becomes progressively more difficult to detect in ground-based spectra for permitted lines weaker than $\mathrm{H} \alpha$. However, HST spectra of LINERs, when available, show broad higher-order Balmer lines as well as UV lines such as Ly $\alpha, \mathrm{C}$ IV $\lambda 1549, \mathrm{Mg}$ II $\lambda 2800$, and Fe II multiplets (Barth et al. 1996; Ho, Filippenko \& Sargent 1996). A subset of LINERs contain broad lines with doublepeaked profiles (Figure 6), analogous to those seen in a minority of radio galaxies (Eracleous \& Halpern 1994), where they are often interpreted as a kinematic signature of a relativistic accretion disk (Chen \& Halpern 1989). Most of the nearby cases have been discovered serendipitously, either as a result of the broad component being variable (NGC 1097: Storchi-Bergmann, Baldwin \& Wilson 1993; M81: Bower et al. 1996; NGC 3065: Eracleous \& Halpern 2001) or because of the increased sensitivity to weak, broad features afforded by small-aperture measurements made with HST (NGC 4450: Ho et al. 2000; NGC 4203: Shields et al. 2000; NGC 4579: Barth et al. 2001a). Double-peaked broad-line AGNs may be more common than previously thought, especially among LLAGNs, perhaps as a consequence of their accretion disk structure $(\S 8)$.

A pressing question, however, is: What fraction of the more numerous LINER $2 \mathrm{~s}$ are AGNs? By analogy with the Seyfert 2 class, do LINER 2s contain a hidden LINER 1 nucleus? At first sight, it might seem that there is no a priori reason 
why the orientation-dependent unification model, which has enjoyed much success in the context of Seyfert galaxies, should not apply equally to LINERs. If we suppose that the ratio of LINER $2 \mathrm{~s}$ to LINER $1 \mathrm{~s}$ is similar to the ratio of Seyfert $2 \mathrm{~s}$ to Seyfert $1 \mathrm{~s}-1.6: 1$ in the Palomar survey-we can reasonably surmise that the AGN fraction in LINERs may be as high as $\sim 60 \%$. That at least some LINERs do indeed contain a hidden BLR was demonstrated by the deep Keck spectropolarimetric observations of Barth, Filippenko \& Moran (1999a, 1999b). In a survey of 14 LLAGNs, mostly LINERs, these authors detected broad $\mathrm{H} \alpha$ emission in three objects $(\sim 20 \%)$ polarized at a level of $1 \%-3 \%$. Interestingly, all three objects are elliptical galaxies with double-sided radio jets. NGC 315 and NGC 1052 technically qualify as type 1.9 LINERs (Ho et al. 1997e), whereas NGC 4261 is a LINER 2. Although the sample is small, these observations prove two important points: (1) the weak broad $\mathrm{H} \alpha$ features detected in direct light is not always scattered emission (Antonucci 1993), since polarized emission was not detected in several other LINER 1.9s included in Barth, Filippenko \& Moran's survey; (2) an obscured nucleus does lurk in some LINER 2s.

At the same time, other bright LINER 2 s have resisted detection by spectropolarimetry. As in the case of Seyferts (Tran 2001), however, the nondetection of polarized broad lines does not necessarily imply that there is no hidden BLR. Nevertheless, the BLR in some type 2 AGNs, especially LINERs but also Seyferts, may be intrinsically absent, not obscured. In the case of some Seyferts, mostly weak sources, the evidence comes from low absorbing X-ray column densities (Bassani et al. 1999; Pappa et al. 2001; Panessa \& Bassani 2002; Gliozzi et al. 2004; Cappi et al. 2006; Gliozzi, Sambruna \& Foschini 2007; Bianchi et al. 2008; but see Ghosh et al. 2007) as well as optical variability (Hawkins 2004). LINERs, as a class, very much conform to this picture. As discussed further below, LINERs of either type generally show very little sign of absorbing or reprocessing material, and UV variability is common. A few exceptions exist (e.g., NGC 1052: Guainazzi et al. 2000; NGC 4261: Sambruna et al. 2003, Zezas et al. 2005), but, interestingly, these are precisely the very ones for which Barth, Filippenko, \& Moran discovered hidden BLRs. NGC 4258, also highly absorbed in the X-rays (Fiore et al. 2001), shows polarized narrow lines rather than broad lines (Barth et al. 1999).

An excellent of a LINER with a naked type 2 nucleus is the Sombrero galaxy. Although clearly an AGN, it shows no trace of a broad-line component, neither in direct light (Ho et al. 1997e), not even when very well isolated with a small HST aperture (Nicholson et al. 1998), nor in polarized light (Barth, Filippenko \& Moran 1999b). Its Balmer decrement indicates little reddening to the NLR. For all practical purposes, the continuum emission from the nucleus looks unobscured. It is detected as a variable UV source (Maoz et al. 1995, 2005) and in the soft and hard X-rays (Nicholson et al. 1998; Ho et al. 2001). The X-ray spectrum is only very mildly absorbed (Nicholson et al. 1998; Pellegrini et al. 2002, 2003a; Terashima et al. 2002), with no signs of Fe K $\alpha$ emission expected from reprocessed material, consistent with the modest mid-IR emission reported by Bendo et al. (2006). In short, there is no sign of anything being hidden or much doing the hiding. So where is the BLR? It is just not there.

The lack of a BLR in very low-luminosity sources may be related to a physical upper limit in the broad-line width (Laor 2003). If LLAGNs obey the same BLR-luminosity relation as in higher luminosity systems, their BLR velocity depends on the $\mathrm{BH}$ mass and luminosity. At a limiting bolometric luminosity of 
$L_{\mathrm{bol}} \approx 10^{41.8}\left(M_{\mathrm{BH}} / 10^{8} M_{\odot}\right)^{2} \operatorname{ergs~s}^{-1}, \Delta v \approx 25,000 \mathrm{~km} \mathrm{~s}^{-1}$, above which clouds may not survive due to excessive shear or tidal forces. Alternatively, if BLR clouds arise from condensations in a radiation-driven, outflowing wind (Murray \& Chiang 1997), a viewpoint now much espoused, then it is reasonable to expect that very low-luminosity sources would be incapable of generating a wind, and hence of sustaining a BLR. For example, the clumpy torus model of Elitzur \& Shlosman (2006) predicts that the BLR can no longer be sustained for $L_{\text {bol }}$ $\lesssim 10^{42} \mathrm{ergs} \mathrm{s}^{-1}$. In the scenario of Nicastro (2000), the BLR originates from a disk outflow formed at the transition radius between regions dominated by gas and radiation pressure. As this radius shrinks with decreasing $L_{\text {bol }} / L_{\text {Edd }}$, where $L_{\mathrm{Edd}}=1.3 \times 10^{38}\left(M_{\mathrm{BH}} / M_{\odot}\right)$ ergs s$^{-1}$, the BLR is expected to disappear for $L_{\mathrm{bol}} / L_{\mathrm{Edd}} \lesssim 10^{-3}$. The apparent correlation between BLR line width and $L_{\text {bol }} / L_{\text {Edd }}$ qualitatively supports this picture (Xu \& Cao 2007). Although the existing data are sparse, they indicate that LINERs generally lack UV resonance absorption features indicative of nuclear outflows (Shields et al. 2002). The models by Elitzur \& Shlosman and Nicastro are probably correct in spirit but not in detail, because many of the Palomar LLAGNs plainly violate their proposed thresholds $(\S 5.10)$.

Nonetheless, the statistics within the Palomar survey already provide tentative support to the thesis that the BLR vanishes at the lowest luminosities or Eddington ratios. Which of the two is the controlling variable is still difficult to say. For both Seyferts and LINERs, type 1 sources are almost a factor of 10 more luminous than type 2 sources in terms of their median total $\mathrm{H} \alpha$ luminosity (Table 1). (The statistical differences between type 1 and type 2 sources cannot be ascribed to sensitivity differences in the detectability of broad $\mathrm{H} \alpha$ emission. Type 1 objects do have stronger line emission compared to the type $2 \mathrm{~s}$, but on average their narrow $\mathrm{H} \alpha$ flux and equivalent width are only $\sim 50 \%$ higher, and the two types overlap significantly. Moreover, as noted in $\S 3.4$, the broad $\mathrm{H} \alpha$ detection rates turn out to be quite robust even in light of the much higher sensitivity afforded by $H S T$.) The differences persist after normalizing by the Eddington luminosities: adopting a bolometric correction of $L_{\mathrm{bol}} \approx 16 L_{\mathrm{X}}, L_{\mathrm{bol}} / L_{\mathrm{Edd}}=1.1 \times 10^{-3}$ and $5.9 \times 10^{-6}$ for Seyfert $1 \mathrm{~s}$ and Seyfert $2 \mathrm{~s}$, respectively, whereas the corresponding values for LINER 1 s and LINER 2 s are $1.0 \times 10^{-5}$ and $4.8 \times 10^{-6}$. Two caveats are in order. First, while most of the type 1 sources have X-ray data, only $60 \%$ of the LINER 2s and $70 \%$ of the Seyfert 2s do. Second, the X-ray luminosities, which pertain to the $2-10 \mathrm{keV}$ band, have been corrected for intrinsic absorption whenever possible, but many sources are too faint for spectral analysis. The lower $\mathrm{X}$-ray luminosities for the type 2 sources must be partly due to absorption, but considering the generally low absorbing columns, particularly among the LINERs (Georgantopoulous et al. 2002; Terashima et al. 2002), it is unclear if absorption alone can erase the statistical difference between the two types. The tendency for Seyfert 2s to have lower Eddington ratios than Seyfert 1s has previously been noted, for the Palomar sample (Panessa et al. 2006) and others (Middleton, Done \& Schurch 2008).

Several authors have raised the suspicion that LINER 2s may not be accretionpowered. Large-aperture X-ray spectra of LINER $2 \mathrm{~s}$, like those of LINER 1s, can be fit with a soft thermal component plus a power law with $\alpha \approx-0.7$ to -1.5 (Georgantopoulos et al. 2002; Terashima et al. 2002). But this alone does not provide enough leverage to distinguish AGNs from starburst galaxies, many of which look qualitatively similar over the limited energy range covered by these 
observations. We cannot turn to the iron $\mathrm{K} \alpha$ line or variability for guidance, because LLAGNs generally exhibit neither $(\S 5.3)$. The hard X-ray emission in LINER 2s is partly extended (Terashima et al. 2000a; Georgantopoulos et al. 2002), but the implications of this finding are unclear. Just because the X-ray emission surrounding the LLAGN is morphologically complex and there is evidence for circumnuclear star formation (e.g., NGC 4736; Pellegrini et al. 2002) does not necessarily imply that there is a causal connection between the starburst and the LLAGN. Roberts, Schurch \& Warwick (2001) advocate a starburst connection from the observation that LINER 2s have a mean flux ratio in the soft and hard X-ray band $(\sim 0.7)$ similar to that found in NGC 253 . This interpretation, however, conflicts with the stellar population constraints discussed in $\S 4.2$. It is also not unique. Luminous, AGN-dominated type 1 sources themselves exhibit a tight correlation between soft and hard X-ray luminosity, with a ratio not dissimilar from the quoted value (Miniutti et al. 2008).

An important clue comes from the fact that many LINER $2 \mathrm{~s}$ have a lower $L_{\mathrm{X}} / L_{\mathrm{H} \alpha}$ ratio than LINER 1s (Ho et al. 2001). In particular, the observed X-ray luminosity from the nucleus, when extrapolated to the UV, does not have enough ionizing photons to power the $\mathrm{H} \alpha$ emission (Terashima et al. 2000a). This implies that (1) the X-rays are heavily absorbed, (2) nonnuclear processes power much of the optical line emission, or (3) the ionizing SED is different than assumed. As discussed in $\S 6.4$, this energy budget discrepancy appears to be symptomatic of all LLAGNs in general, not just LINER $2 \mathrm{~s}$, and most likely results from a combination of the second and third effect. There are some indications that the SEDs of LINER $2 \mathrm{~s}$ indeed differ systematically from those of LINER 1s (e.g., Maoz et al. 2005; Sturm et al. 2006). In light of the evidence given in $\S \S 5.3$, 5.6, I consider the first solution to be no longer tenable. One can point to objects such as NGC 4261 (Zezas et al. 2005) as examples of LINER 2s with strong obscuration, but such cases are rare.

From the point of view of $\mathrm{BH}$ demographics, the most pressing issue is what fraction of the LINER 2s should be included in the AGN tally. Some cases are beyond dispute (M84, M87, Sombrero). What about the rest? The strongest argument that the majority of LINER 2s are AGN-related comes from the detection frequency of radio ( $(5.2)$ and X-ray ( $\S 5.3$ ) cores, which is roughly $60 \%$ of that of LINER 1s. On the other hand, the detection rate of Seyfert $2 \mathrm{~s}$ are similarly lower compared to Seyfert 1s, most likely reflecting the overall reduction of nuclear emission across all bands in type 2 LLAGNs as a consequence of their lower accretion rates. In summary, the AGN fraction among LINER 2s is at least $60 \%$, and possibly as high as $100 \%$.

\subsection{Torus}

In line with the absence of a BLR discussed above and using very much the same set of evidence, a convincing case can be made that the torus also disappears at very low luminosities. In a large fraction of nearby LINERs, the low absorbing column densities and weak or undetected Fe K $\alpha$ emission ( $§ 5.3)$ strongly indicate that we have a direct, unobstructed view of the nucleus. Ghosh et al. (2007) warn that absorbing columns can be underestimated in the presence of extended soft emission, especially when working with spectra of low signal-to-noise ratio. While this bias no doubt enters at some level, cases like the Sombrero ( $\$ 5.3)$ cannot be so readily dismissed. By analogy with situation in luminous AGNs (e.g., Inoue, 
Terashima \& Ho 2007; Nandra et al. 2007), type 1 LLAGNs, if they possess tori, should also show strong, narrow fluorescent Fe $\mathrm{K} \alpha$ emission. This expectation is not borne out by observations. NGC 3998, which has excellent X-ray data, offers perhaps the most dramatic example. Apart from showing no signs whatsoever for intrinsic photoelectric absorption, it also possesses one of the tightest upper limits to date on $\mathrm{Fe} \mathrm{K} \alpha$ emission: $\mathrm{EW}<25 \mathrm{eV}$ (Ptak et al. 2004). Our sight line to the nucleus is as clean as a whistle. Satyapal, Sambruna \& Dudik (2004) claim that many LINERs have obscured nuclei, but this conclusion is based on IR-bright, dusty objects chosen from Carrillo et al. (1999); as I have discussed in $\S 3.2$, I regard these objects not only as biased, but also confusing with respect to their nuclear properties.

Palomar Seyferts, whose luminosities and Eddington ratios are about an order of magnitude higher than those of LINERs ( $(5.10)$, show markedly larger absorbing column densities and stronger Fe $\mathrm{K} \alpha$ lines. In an XMM-Newton study of a distance-limited sample of 27 Palomar Seyferts, Cappi et al. (2006) detect strong $\mathrm{Fe} \mathrm{K} \alpha$ emission in over half of objects. The distribution of absorbing columns is nearly continuous, from $N_{\mathrm{H}} \approx 10^{20}$ to $10^{25} \mathrm{~cm}^{-2}$, with $30 \%-50 \%$ of the type 2 sources being Compton-thick (Panessa et al. 2006). This seems consistent with the tendency for Seyferts to be more gas-rich than LINERs, to the extent that this is reflected in their higher NLR densities (Ho, Filippenko \& Sargent 2003).

The trend of increasing absorption with increasing luminosity or Eddington ratio observed in Palomar LLAGNs has an interesting parallel among radio galaxies. A substantial body of recent work indicates that the nuclei of FR I sources, most of which are, in fact, LINERs, are largely unobscured (e.g., Chiaberge, Capetti \& Celotti 1999; Donato, Sambruna \& Gliozzi 2004; Balmaverde \& Capetti 2006). In contrast, FR II systems, especially those with broad or high-excitation lines (analogs of Seyferts), show clear signs of absorption and $\mathrm{Fe} \mathrm{K} \alpha$ emission (Evans et al. 2006).

Even if we are fooled by the X-ray observations, substantial absorption must result in strong thermal reemission of "waste heat" in the IR. While sources such as Cen A provide a clear reminder that every rule has its exception (Whysong \& Antonucci 2004), the existing data do suggest that, as a class, FR I radio galaxies tend to be weak mid-IR or far-IR sources (Haas et al. 2004; Müller et al. 2004). The same holds for more nearby LINERs. Their SEDs do show a pronounced mid-IR peak ( $\S 5.8)$, but as I will argue later, it is due to emission from the accretion flow rather than from dust reemission.

\subsection{Narrow-line Region Kinematics}

The kinematics of the NLR are complex. At the smallest scales probed by HST, Verdoes Kleijn, van der Marel \& Noel-Storr (2006) find that the velocity widths of the ionized gas in the LINER nuclei of early-type galaxies can be modeled as unresolved rotation of a thin disk in the gravitational potential of the central $\mathrm{BH}$. The subset of objects with FR I radio morphologies, on the other hand, exhibit line broadening in excess of that expected from purely gravitational motions; these authors surmise that the super-virial motions may be related to an extra source of energy injection by the radio jet. Walsh et al. (2008) use multiple-slit STIS observations to map the kinematics of the inner $\sim 100 \mathrm{pc}$ of the NLR in a sample of 14 LLAGNs, mostly LINERs. Consistent with earlier findings (Ho et al. 2002; Atkinson et al. 2005), the velocity fields are generally quite disorganized, rarely 
showing clean signatures of dynamically cold disks undergoing circular rotation. Nevertheless, two interesting trends can be discerned. The emission line widths tend to be largest within the sphere of influence of the $\mathrm{BH}$, progressively decreasing toward large radii to values that roughly match the stellar velocity dispersion of the bulge. The luminous members of the sample, on the other hand, show more chaotic kinematics, as evidenced by large velocity splittings and asymmetric line profiles, reminiscent of the pattern observed by Rice et al. (2006) in their sample of Seyfert galaxies. Walsh et al. suggest that above a certain luminosity threshold - one that perhaps coincides with the LINER/Seyfert division-AGN outflows and radio jets strongly perturb the kinematics of the NLR.

A large fraction $(\sim 90 \%)$ of the Palomar LLAGNs have robust measurements of integrated [N II] $\lambda 6583$ line widths, which enable a crude assessment of the dynamical state of the NLR and its relation to the bulge. Consistent with what has been established for more powerful systems (Nelson \& Whittle 1996; Greene \& Ho 2005a), the kinematics of the ionized gas are dominated by random motions that, to first order, trace the gravitational potential of the stars in the bulge. Among the objects with available central stellar velocity dispersions, $\sigma_{\mathrm{NLR}} / \sigma_{*} \approx$ $0.7-0.8$ for the weakest sources $\left(L_{\mathrm{H} \alpha} \approx 10^{38} \mathrm{ergs} \mathrm{s}^{-1}\right)$, systematically rising to $\sigma_{\mathrm{NLR}} / \sigma_{*} \approx 1.2$ in the more luminous members $\left(L_{\mathrm{H} \alpha} \approx 10^{41.5} \mathrm{ergs} \mathrm{s}^{-1}\right)$. L.C. Ho (in preparation) speculates that the central AGN injects a source of dynamical heating of nongravitational origin to the NLR, either in the form of radiation pressure from the central continuum or mechanical interaction from radio jets. Given the empirical correlation between optical line luminosity and radio power (e.g., Ho \& Peng 2001; Ulvestad \& Ho 2001a; Nagar, Falcke \& Wilson 2005), and the near ubiquity of compact radio sources, it is a priori difficult to determine which of these two sources acts as the primary driver. The tendency for extended radio emission to be more prevalent in Seyferts ( $\S 5.2$ ) suggests that jets may be more important.

\subsection{Spectral Energy Distribution}

The broad-band SED provides one of the most fundamental probes of the physical processes in AGNs. Both thermal and nonthermal emission contribute to the broad-band spectrum of luminous AGNs such as quasars and classical Seyfert galaxies. In objects whose intrinsic spectrum has not been modified severely by relativistic beaming or absorption, the SED can be separated into several distinctive components (e.g., Elvis et al. 1994): radio synchrotron emission from a jet, which may be strong ("radio-loud") or weak ("radio-quiet"); an IR excess, now generally considered to be predominantly thermal reradiation by dust grains; a prominent optical to UV "big blue bump," usually interpreted to be pseudoblackbody emission from an optically thick, geometrically thin accretion disk (Shields 1978; Malkan \& Sargent 1982); a soft X-ray excess, whose origin is still highly controversial (Done et al. 2007; Miniutti et al. 2008); and an underlying power law, which is most conspicuous at hard X-ray energies but is thought to extend down to IR wavelengths, that can be attributed to Comptonization of softer seed photons.

Within this backdrop, there were already early indications that the SEDs of LINERs may deviate from the canonical form. Halpern \& Filippenko (1984) succeeded in detecting the featureless optical continuum in NGC 7213, and while these authors suggested that a big blue bump may be present in this object, they 
also noted that it possesses an exceptionally high X-ray-to-optical flux ratio, although perhaps one not inconsistent with the extrapolation of the trend of increasing X-ray-to-optical flux ratio with decreasing luminosity seen in luminous sources (Zamorani et al. 1981; Avni \& Tananbaum 1982). A more explicit suggestion that LINERs may possess a weak UV continuum was made in the context of double-peaked broad-line AGNs such as Arp 102B and Pictor A, whose narrow-line spectra share many characteristics with LINERs (Chen \& Halpern 1989; Halpern \& Eracleous 1994). The HST spectrum of Arp 102B, in fact, shows an exceptionally steep optical-UV nonstellar continuum $(\alpha \approx-2.1$ to -2.4; Halpern et al. 1996). Halpern \& Eracleous (1994) further suggested that the SEDs are flat in the far-IR. In an important study of M81, Petre et al. (1993) proposed that the relative weakness of the UV continuum compared to the X-rays is a consequence of a change in the structure of the central accretion flow, from a standard thin disk to an ion-supported torus (see $\S 8.3$ ). Parameterizing the two-point spectral index between $2500 \AA$ and $2 \mathrm{keV}$ by $\alpha_{\text {ox }} \equiv\left[\log L_{\nu}(2500 \AA)-\right.$ $\left.\log L_{\nu}(2 \mathrm{keV})\right] /[\log \nu(2500 \AA)-\log \nu(2 \mathrm{keV})]$, M81 and possibly other LINERs (Mushotzky 1993) have $\alpha_{\text {ox }} \gtrsim-1$, to be compared with $\alpha_{\text {ox }} \approx-1.4$ for quasars and $\alpha_{\mathrm{ox}} \approx-1.2$ for Seyferts (Mushotzky \& Wandel 1989).

The full scope of the spectral uniqueness of LLAGNs only became evident once the modern, albeit still fragmentary, multiwavelength data could be assembled. The initial studies concentrated on individual objects, emphasizing the weakness of the UV bump (M81: Ho, Filippenko \& Sargent 1996; Sombrero: Nicholson et al. 1998) and the overall consistency of the SED with spectral models generated from advection-dominated accretion flows (ADAFs; see Narayan 2002 and Yuan 2007 for reviews) as unique attributes of systems with low Eddington ratios (NGC 4258: Lasota et al. 1996, Chary et al. 2000; M87: Reynolds et al. 1996; M60: Di Matteo \& Fabian 1997). Ho (1999b) systematically investigated the SEDs of a small sample of seven LLAGNs with available BH mass estimates and reliable small-aperture fluxes from radio to X-ray wavelengths. This was followed by a study of another five similar objects, which have the additional distinction of having double-peaked broad emission lines (Ho et al. 2000; Ho $2002 \mathrm{~b})$. Figure 7 gives the latest update from a comprehensive analysis of the SEDs of 150 nearby type 1 AGNs spanning 4 dex in $\mathrm{BH}$ mass $\left(M_{\mathrm{BH}} \approx 10^{5}-10^{9}\right.$ $\left.M_{\odot}\right)$ and 6.5 dex in Eddington ratio $\left(L_{\mathrm{bol}} / L_{\mathrm{Edd}} \approx 10^{-6}-10^{0.5}\right)$. Let us focus on two regimes: $L_{\mathrm{bol}} / L_{\mathrm{Edd}}=0.1$ to 1 , typical of classical, luminous AGNs, and $L_{\text {bol }} / L_{\text {Edd }}<10^{-3.0}$, which characterizes most nearby LLAGNs $(\S 5.10)$. I defer the discussion of the physical implications until $\S 8$, but for now list the most notable features concerning the LLAGN SED, some of which are also apparent in the composite LINER SED assembled by Eracleous, Hwang \& Flohic (2008a). (1) The big blue bump is conspicuously absent. (2) Instead, a broad excess is shifted to the mid-IR, forming a "big red bump"; this component is probably related to the mid-IR excess previously noted by Lawrence et al. (1985), Willner et al. (1985), and Chen \& Halpern (1989), and more recently from Spitzer observations (e.g., Willner et al. 2004; Bendo et al. 2006; Gu et al. 2007). (3) As a consequence of this shift, the optical-UV slope is exceptionally steep, generally in the range $\alpha_{\mathrm{ou}} \approx-1$ to -2.5 , to be compared with $\alpha_{\mathrm{ou}} \approx-0.5$ to -0.7 for luminous AGNs (Vanden Berk et al. 2001; Shang et al. 2005); the X-ray-to-optical ratio is large, resulting in $\alpha_{\text {ox }} \gtrsim-1$. (4) There is no evidence for a soft X-ray excess. (5) Lastly, the overall SED can be considered radio-loud, defined here by the convention that the radio-to-optical luminosity ratio exceeds a value 


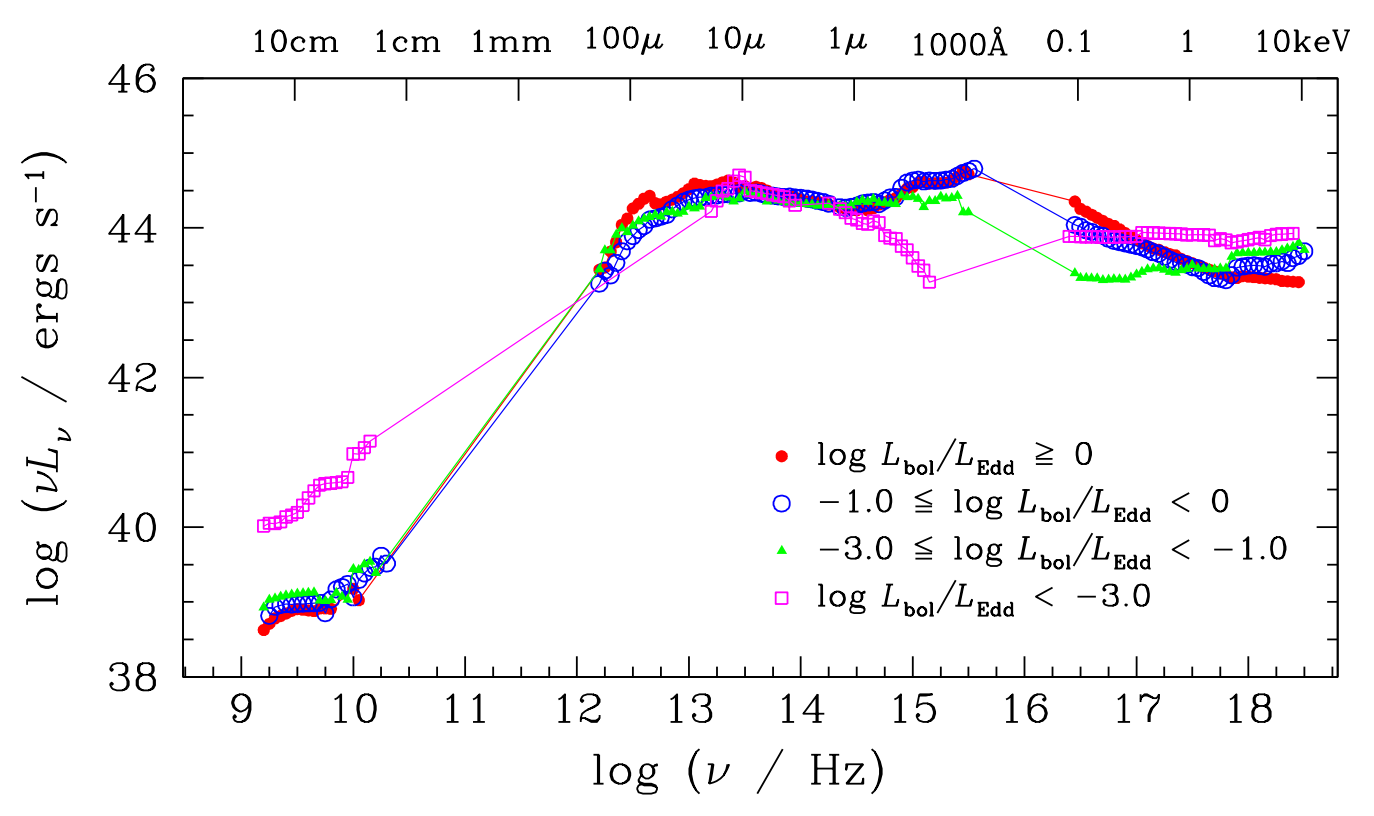

Figure 7: Composite SEDs for radio-quiet AGNs binned by Eddington ratio. The SEDs are normalized at $1 \mu \mathrm{m}$. (Adapted from L.C. Ho, in preparation.)

of $R_{o} \equiv L_{\nu}(5 \mathrm{GHz}) / L_{\nu}(B)=10$. Radio-loudness, in fact, seems to be a property common to essentially all nearby weakly active nuclei (Ho 1999b, 2002a; Ho et al. 2000) and a substantial fraction of Seyfert nuclei (Ho \& Peng 2001). Defining radio-loudness based on the relative strength of the radio and X-ray emission, $R_{\mathrm{X}} \equiv \nu L_{\nu}(5 \mathrm{GHz}) / L_{\mathrm{X}}$, Terashima \& Wilson (2003b) also find that LINERs tend to be radio-loud, here taken to be $R_{\mathrm{X}}>10^{-4.5}$. Moreover, the degree of radio-loudness scales inversely with $L_{\text {bol }} / L_{\text {Edd }}$ (Ho 2002a; Terashima \& Wilson 2003b; Wang, Luo \& Ho 2004; Greene, Ho \& Ulvestad 2006; Panessa et al. 2007; Sikora, Stawarz \& Lasota 2007; L.C. Ho, in preparation; see Figure 10b).

In a parallel development, studies of the low-luminosity, often LINER-like nuclei of FR I radio galaxies also support the notion that they lack a UV bump. M84 (Bower et al. 2000) and M87 (Sabra et al. 2003) are two familiar examples, but it has been well documented that FR I nuclei tend to exhibit flat $\alpha_{\mathrm{ox}}$ (Donato, Sambruna \& Gliozzi 2004; Balmaverde, Capetti \& Grandi 2006; Gliozzi et al. 2008) and steep slopes in the optical (Chiaberge, Capetti \& Celotti 1999; Verdoes Kleijn et al. 2002) and optical-UV (Chiaberge et al. 2002).

Finally, I note that the UV spectral slope can be indirectly constrained from considering the strength of the He II $\lambda 4686$ line. While this line is clearly detected in Pictor A (Carswell et al. 1984; Filippenko 1985), its weakness in NGC 1052 prompted Péquignot (1984) to deduce that the ionizing spectrum must show a sharp cutoff above the $\mathrm{He}^{+}$ionization limit $(54.4 \mathrm{eV})$. In this respect, NGC 1052 is quite representative of LINERs in general. He II $\lambda 4686$ was not detected convincingly in a single case among a sample of 159 LINERs in the entire Palomar survey (Ho, Filippenko \& Sargent 1997a). Starlight contamination surely contributes partly to this, but the line has also eluded detection in $H S T$ spectra (e.g., 
Ho, Filippenko \& Sargent 1996; Nicholson et al. 1998; Barth et al. 2001b; Sabra et al. 2003; Sarzi et al. 2005; Shields et al. 2007), which indicates that it is truly intrinsically very weak. To a first approximation, the ratio of $\mathrm{He}$ II $\lambda 4686$ to $\mathrm{H} \beta$ reflects the relative intensity of the ionizing continuum between 1 and 4 Ryd. For an ionizing spectrum $f_{\nu} \propto \nu^{\alpha}$, case B recombination predicts He II $\lambda 4686 / \mathrm{H} \beta=$ $1.99 \times 4^{\alpha}$ (Penston \& Fosbury 1978). The current observational limits of He II $\lambda 4686 / \mathrm{H} \beta \lesssim 0.1$ thus imply $\alpha \lesssim-2$, qualitatively consistent with the evidence from the SED studies.

Maoz (2007) has offered an alternative viewpoint to the one presented above. Using a sample of 13 LINERs with variable UV nuclei, he argues that their SEDs do not differ appreciably from those of more luminous AGNs, and hence that LINERs inherently have very similar accretion disks compared to powerful AGNs. Maoz does not disagree that LINERs have large X-ray-to-UV flux ratios or that they tend to be radio-loud; his data show both trends. Rather, he contends that because LINERs lie on the low-luminosity extrapolation of the well-known relation between $\alpha_{\text {ox }}$ and luminosity (Zamorani et al. 1981; Avni \& Tananbaum 1982; Strateva et al. 2005) they do not form a distinct population. And while LINERs do have large values of $R_{o}$, they nonetheless occupy the "radio-quiet" branch of the $R_{o}$ versus $L_{\text {bol }} / L_{\text {Edd }}$ plane (Sikora, Stawarz \& Lasota 2007). In my estimation, the key point is not, and has never been, whether LINERs constitute a disjoint class of AGNs, but whether they fit into a physically plausible framework in which their distinctive SEDs, among other properties, find a natural, coherent explanation. Section 8 attempts to offer such a framework.

It should be noted that Maoz's results strongly depend on his decision to exclude all optical and near-IR data from the SEDs, on the grounds that they may be confused by starlight. I think this step is too draconian, as it throws away valuable information. While stellar contamination is certainly a concern, one can take necessary precautions to try to isolate the nuclear emission as much as possible, either through high-resolution imaging (e.g., Ho \& Peng 2001; Ravindranath et al. 2001; Peng et al. 2002) or spectral decomposition. In well-studied sources, there is little doubt that the optical continuum is truly both featureless and nonstellar (e.g., Halpern \& Filippenko 1984; Ho, Filippenko \& Sargent 1996; Ho et al. 2000; Bower et al. 2000; Sabra et al. 2003). Given what we know about the nuclear stellar population, we cannot assign the featureless continuum to young stars. In a few cases, the nonstellar nature of the nucleus can even be established through variability in the optical (Bower et al. 2000; Sabra et al. 2003; O'Connell et al. 2005) and mid-IR (Rieke, Lebofsky \& Kemp 1982; Grossan et al. 2001; Willner et al. 2004).

While the SEDs of LINERs differ from those of traditional AGNs, it is important to recognize that they are decidedly nonstellar and approximate the form predicted for radiatively inefficient accretion flows (RIAFs) onto BHs, often coupled to a jet (Quataert et al. 1999; Yuan, Markoff \& Falcke 2002; Yuan et al. 2002; Fabbiano et al. 2003; Pellegrini et al. 2003b; Ptak et al. 2004; Nemmen et al. 2006; Wu, Yuan \& Cao 2007). They bear little resemblance to SEDs characteristic of normal stellar systems. Inactive galaxies or starburst systems not strongly affected by dust extinction emit the bulk of their radiation in the optical-UV and in the thermal IR regions, with only an energetically miniscule contribution from X-rays. 


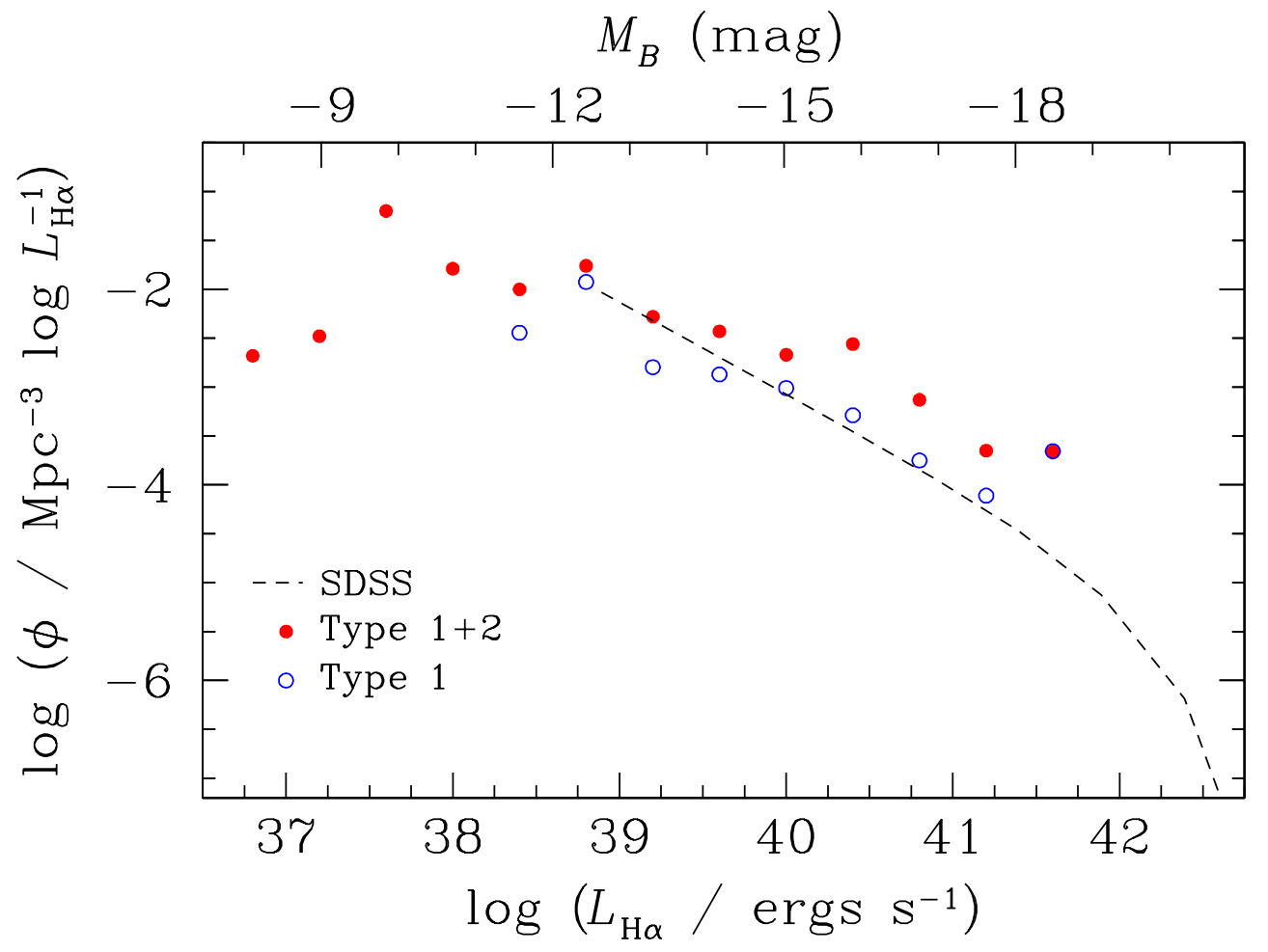

Figure 8: The $\mathrm{H} \alpha$ nuclear luminosity function of nearby AGNs derived from the Palomar survey. The top axis gives an approximate conversion to absolute magnitudes in the $B$ band, using the $\mathrm{H} \alpha$-continuum correlation of Greene \& Ho (2005b). The unfilled circles include only type 1 sources, while the filled circles represent both type 2 and type 1 sources. The luminosities have been corrected for extinction, and in the case of type 1 nuclei, they include both the narrow and broad components of the line. For comparison, I show the $z<0.35$ luminosity function for SDSS Seyfert galaxies (types 1 and 2; dashed line; Hao et al. 2005b). (Adapted from L.C. Ho, A.V. Filippenko \& W.L.W. Sargent, in preparation.)

\subsection{Luminosity Function}

Many astrophysical applications of AGN demographics benefit from knowing the AGN luminosity function, $\Phi(L, z)$. Whereas $\Phi(L, z)$ has been reasonably well charted at high $L$ and high $z$ using quasars, it is very poorly known at low $L$ and low $z$. Indeed, until very recently there has been no reliable determination of $\Phi(L, 0)$. The difficulty in determining $\Phi(L, 0)$ can be ascribed to a number of factors, as discussed in Huchra \& Burg (1992). First and foremost is the challenge of securing a reliable, spectroscopically selected sample. Since nearby AGNs are expected to be faint relative to their host galaxies, most of the traditional techniques used to identify quasars cannot be applied without introducing large biases. The faintness of nearby AGNs presents another obstacle, namely how to disentangle the nuclear emission - the only component relevant to the AGNfrom the usually much brighter contribution from the host galaxy. Finally, most optical luminosity functions of bright, more distant AGNs are specified in terms of the nonstellar optical continuum (usually the $B$ band), whereas spectroscopic 
surveys of nearby galaxies generally only reliably measure optical line emission (e.g., $\mathrm{H} \alpha$ ) because the featureless nuclear continuum is often impossible to detect in ground-based, seeing-limited apertures.

A different strategy can be explored by taking advantage of the fact that $\mathrm{H} \alpha$ luminosities are now available for nearly all of the AGNs in the Palomar survey. Figure 8 shows the $\mathrm{H} \alpha$ luminosity function for the Palomar sources, computed using the $V / V_{\max }$ method (L.C. Ho, A.V. Filippenko \& W.L.W. Sargent, in preparation). Two versions are shown, each representing an extreme view of what kind of sources should be regarded as bona fide AGNs. The open symbols include only type 1 nuclei, whose AGN status is incontrovertible. This may be regarded as the most conservative assumption and a lower bound, since we know that genuine narrow-line AGNs do exist. The filled symbols lump together all sources classified as LINERs, transition objects, and Seyferts, both type 1 and type 2 . This represents the most optimistic view and an upper bound, if some type 2 sources are in fact AGN impostors, although, as I argue in $\S 6.5$, this is likely to be a small effect. The true space density of local AGNs lies between these two possibilities. In either case, the differential luminosity function can be approximated by a single power law from $L_{\mathrm{H} \alpha} \approx 10^{38}$ to $3 \times 10^{41} \mathrm{ergs} \mathrm{s}^{-1}$, roughly of the form $\Phi \propto L^{-1.2 \pm 0.2}$. The slope seems to flatten below $L_{\mathrm{H} \alpha} \approx 10^{38} \mathrm{ergs} \mathrm{s}^{-1}$, but the luminosity function is highly uncertain at the faint end because of density fluctuations in our local volume. Nevertheless, it is remarkable that the Palomar luminosity function formally begins at $L_{\mathrm{H} \alpha} \approx 6 \times 10^{36} \mathrm{ergs} \mathrm{s}^{-1}$, roughly the luminosity of the Orion nebula (Kennicutt 1984). In units more familiar to the AGN community, this corresponds to an absolute $B$-band magnitude of roughly -8 (using the $\mathrm{H} \alpha$-optical continuum conversion of Greene \& Ho 2005b), no brighter than a single supergiant star.

For comparison, I have overlaid the $\mathrm{H} \alpha$ luminosity function of $z \lesssim 0.35$ Seyfert galaxies derived from the SDSS by Hao et al. (2005b). The Palomar survey reaches $\sim 2$ orders of magnitude fainter in $\mathrm{H} \alpha$ luminosity than SDSS, but the latter extends a factor of 10 higher at the bright end. Over the region of overlap, the two surveys show reasonably good agreement, especially considering the small number statistics of the Palomar survey and the fact that Hao et al.'s sample only includes Seyferts.

\subsection{Bolometric Luminosities and Eddington Ratios}

To gain further insight into the physical nature of LLAGNs, it is more instructive to examine their bolometric luminosities rather than their luminosities in a specific band or emission line. Because AGNs emit a very broad spectrum, their bolometric luminosities ideally should be measured directly from their full SEDs. In practice, however, complete SEDs are not readily available for most AGNs, and one commonly estimates $L_{\text {bol }}$ by applying bolometric corrections derived from a set of well-observed calibrators. As discussed in $\S 5.8$, the SEDs of LLAGNs differ quite markedly from those of conventionally studied AGNs. Nonetheless, they do exhibit a characteristic shape, which enables bolometric corrections to be calculated. The usual practice of choosing the optical $B$ band as the reference point should be abandoned for LLAGNs, not only because reliable optical continuum measurements are scarce but also because the optical/UV region of the SED shows the maximal variance with respect to accretion rate $(\S 5.8)$ and depends sensitively on extinction. What is available, by selection, is nuclear emission-line 

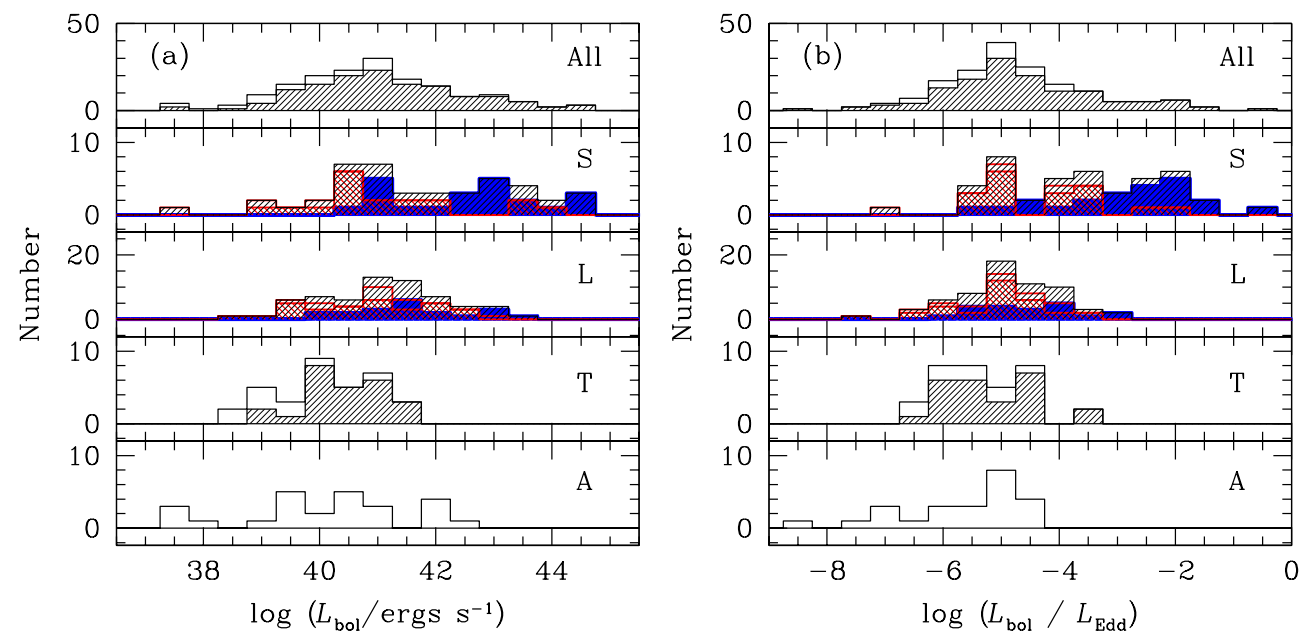

Figure 9: Distribution of $(a)$ bolometric luminosity, $L_{\mathrm{bol}}$, and $(b)$ ratio of bolometric luminosity to the Eddington luminosity, $L_{\mathrm{bol}} / L_{\mathrm{Edd}}$, for all objects, Seyferts (S), LINERs (L), transition objects (T), and absorption-line nuclei (A). $L_{\text {bol }}$ is based on the X-ray $(2-10 \mathrm{keV})$ luminosity. The hatched and open histograms denote detections and upper limits, respectively; type 1 objects are plotted in blue, type 2 objects in red. (Adapted from L.C. Ho, in preparation.)

fluxes, and upper limits thereon. Although the $\mathrm{H} \alpha$ luminosity comprises only a small percentage of the total power, its fractional contribution to $L_{\text {bol }}$ turns out to be fairly well defined: from the SED study of L.C. Ho (in preparation), $L_{\mathrm{bol}} \approx 220 L_{\mathrm{H} \alpha}$, with an rms scatter of $\sim 0.4 \mathrm{dex}$, consistent with the calibration given in Greene \& Ho (2005b, 2007a). Because of the wide range of ionization levels among LLAGNs, a bolometric correction based on $\mathrm{H} \alpha$ should be more stable than one tied to [O III] $\lambda 5007$ (e.g., Heckman et al. 2005). Nevertheless, in light of the nonnuclear component of the nebular flux in LLAGNs $(\S 6.4)$, the luminosity of the narrow $\mathrm{H} \alpha$ line will tend to overestimate $L_{\mathrm{bol}}$. I recommend that, whenever possible, $L_{\text {bol }}$ should be based on the hard X-ray $(2-10 \mathrm{keV})$ luminosity, bearing in mind the added complication that the bolometric correction in this band is luminosity-dependent. Making use again of the database from L.C. Ho (in preparation), I estimate $L_{\mathrm{bol}} / L_{\mathrm{X}} \approx 83,28$, and 16 for quasars, luminous Seyferts, and LLAGNs, respectively.

Figure 9 shows the distributions of $L_{\mathrm{bol}}$ and their values normalized with respect to the Eddington luminosity for Palomar galaxies with measurements of $L_{\mathrm{X}}$ and central stellar velocity dispersion. The $M_{\mathrm{BH}}-\sigma$ relation of Tremaine et al. (2002) was used to obtain $L_{\mathrm{Edd}}$. Although there is substantial overlap, the four spectral classes clearly delineate a luminosity sequence, with $L_{\text {bol }}$ decreasing systematically as $\mathrm{S} \rightarrow \mathrm{L} \rightarrow \mathrm{T} \rightarrow \mathrm{A}$. The differences become even more pronounced in terms of $L_{\mathrm{bol}} / L_{\mathrm{Edd}}$, with Seyferts having a median value $\left(1.3 \times 10^{-4}\right) 20$ times higher than in LINERs $\left(5.9 \times 10^{-6}\right)$, which in turn are higher than transition objects by a factor of $\sim 5$. Among Seyferts and LINERs, type 1 sources are systematically more luminous than type 2 s. Notably, the vast majority of nearby nuclei have highly sub-Eddington luminosities. The total distribution of Eddington ratios is characterized by a prominent peak at $L_{\mathrm{bol}} / L_{\mathrm{Edd}} \approx 10^{-5}$ dominated by Seyfert 2s, LINERs, and transition objects, and a precipitous drop toward 
larger Eddington ratios. Contrary to previous claims (Wu \& Cao 2005; Hopkins \& Hernquist 2006) based on the smaller sample of Ho (2002a), the distribution of Eddington ratios shows no bimodality. The systematic difference in Eddington ratios between LINERs and Seyferts has been noticed before in the Palomar survey (Ho 2002b, 2003, 2005) and in SDSS (Kewley et al. 2006), but this is the first time that the more subtle differences among the different subclasses can be discerned.

\section{EXCITATION MECHANISMS}

\subsection{Nonstellar Photoionization}

The origin and excitation of the ionized gas in the central regions of nearby galaxies has been a longstanding problem (Minkowski \& Osterbrock 1959; Osterbrock 1960). Ever since the early suggestion of Ferland \& Netzer (1983) and Halpern \& Steiner (1983), photoionization by a central AGN has surfaced as the leading candidate for the excitation mechanism of LINERs. Given the success with which more luminous sources have been explained within this framework, and the growing realization that $\mathrm{BHs}$ are commonplace, extending photoionization models to LINERs is both natural and appealing. The requisite relative strengths of the low-ionization lines in LINERs can be achieved by lowering the ionization parameter, commonly defined as $U=Q_{\text {ion }} / 4 \pi r^{2} c n$, where $Q_{\text {ion }}$ is the number of photons $\mathrm{s}^{-1}$ capable of ionizing hydrogen, $r$ is the distance of the inner face of the cloud from the central continuum, $n$ the hydrogen density, and $c$ the speed of light. While Seyfert line ratios can be matched with $\log U \approx-2 \pm 0.5$ (e.g., Ferland \& Netzer 1983; Stasińska 1984; Ho, Shields \& Filippenko 1993), LINERs require $\log U \approx-3.5 \pm 0.5$ (Ferland \& Netzer 1983; Halpern \& Steiner 1983; Péquignot 1984; Binette 1985; Ho, Filippenko \& Sargent 1993; Groves, Dopita \& Sutherland 2004).

An issue that has not been properly addressed is which of the primary variables - $Q_{\text {ion }}, r$, or $n$ - conspire to reduce $U$ in LINERs to the degree required by the models. The answer seems to be all three. The dominant factor comes from the luminosity, as LINERs emit an order of magnitude less ionizing luminosity than do Seyferts: $\left\langle L_{\mathrm{H} \alpha}\right\rangle=3 \times 10^{39} \mathrm{ergs} \mathrm{s}^{-1}$ versus $29 \times 10^{39} \mathrm{ergs} \mathrm{s}^{-1}$ (Ho, Filippenko $\&$ Sargent 2003). Given the gas-poor environments of LINERs (see $\S 5.6$ ), we can now say with some certainty that the reduction in ionizing luminosity is intrinsic and not due to obscuration (as proposed by Halpern \& Steiner 1983). But this is unlikely to be the end of the story. The electron density of LINERs $\left(\left\langle n_{e}\right\rangle \approx 280\right.$ $\left.\mathrm{cm}^{-3}\right)$, at least as probed by the relatively low-density tracer [S II] $\lambda \lambda 6716,6731$, is $\sim 50 \%$ lower than in Seyferts $\left(\left\langle n_{e}\right\rangle \approx 470 \mathrm{~cm}^{-3}\right)$. Very little is known about the detailed morphology and spatial distribution of the NLR in LINERs, or for that matter in low-luminosity Seyferts. Whereas the NLR in luminous Seyferts span $\sim 50-1000 \mathrm{pc}$ in radius, scaling roughly as $L^{0.5}$ (Bennert et al. 2006), LINERs seem to be significantly more compact. This is perhaps not surprising, if the NLR size-luminosity relation extends to LLAGNs. At typical ground-based resolution, narrow-band imaging studies find that the ionized gas in LINERs tends to be quite centrally peaked, with typical dimensions of $r \lesssim 50-100 \mathrm{pc}$ (Keel 1983a; Pogge 1989). In the instances where narrow-band images or slit spatial profiles are available from HST (Bower et al. 2000; Pogge et al. 2000; Cappellari et al. 2001; Verdoes Kleijn et al. 2002; González Delgado et al. 2004; 
Walsh et al. 2008), the line-emitting gas appears even more concentrated still, with scales $\lesssim$ tens pc, although some of it clearly extends to scales of at least $\sim 200$ pc (Shields et al. 2007). The covering factor is high, on average $\sim 0.3$ for the LINER nuclei of the radio galaxies studied by Capetti, Verdoes Kleijn \& Chiaberge (2005). With very few exceptions (e.g., NGC 1052; Pogge et al. 2000), extended, elongated structures analogous to classical ionization cones in Seyferts do not exist in LINERs. Interestingly, both of these trends (smaller $n_{e}$ and $r$ ) would naively drive $U$ in the opposite direction needed to explain LINERs. On the other hand, we know that the NLRs of AGNs in general, and perhaps of LINERs especially, contain a wide range of densities not probed by [S II] and that this material is highly stratified radially (Wilson 1979; Pelat, Alloin \& Fosbury 1981; Carswell et al. 1984; Filippenko \& Halpern 1984; Péquignot 1984; Binette 1985; Filippenko 1985; Ho, Filippenko \& Sargent 1993, 1996; Barth et al. 2001b; Laor 2003; Shields et al. 2007; Walsh et al. 2008). The effective ionization parameter, therefore, depends on the detailed spatial distribution of the gas.

Whereas basic single-zone photoionization models can match many of the strong lines, it is well-known that more complex, multi-component models, especially ones that incorporate a range of densities, are required to achieve a satisfactory fit (Binette 1985; Gabel et al. 2000; Sabra et al. 2003). A notorious deficiency of single-zone models has been their inability to reproduce the high values of the temperature-sensitive ratio [O III] $\lambda 4363 /[\mathrm{O}$ III] $\lambda 5007$. However, because of the different critical densities of these two transitions, they need not originate cospatially, making [O III $] \lambda 4363 /[\mathrm{O}$ III] $\lambda 5007$ no longer a valid thermometer. The discovery that these two lines indeed have different line widths removed one of the principal objections to photoionization models (Filippenko \& Halpern 1984; Filippenko 1985).

The observed weakness of He II $\lambda 4686 / \mathrm{H} \beta$ (see also $\S 5.8$ ) has also been a thorny problem. Péquignot (1984) achieved a consistent fit to the spectrum of NGC 1052 by invoking a modified ionizing spectrum consisting of an 80,000 K blackbody coupled with an X-ray tail extending to higher energies. The main difficulty with this proposal is that the observed SEDs of LINERs do not have a blackbody component peaked in the UV, nor is Péquignot's specific model unique because Gabel et al. (2000) achieved an equally good - if not betterfit using a simple power-law continuum with $\alpha=-1.2$. Since we now have ample evidence that the SEDs of LINERs are not the same as those of more luminous AGNs, future photoionization calculations should adopt empirically motivated input spectra. Important steps in these directions have been taken (e.g., Nicholson et al. 1998; Gabel et al. 2000; Nagao et al. 2002; Lewis, Eracleous \& Sambruna 2003), but much more can be done. One fruitful avenue to pursue is to incorporate the full observed SED, which is noteworthy not only because of its hard ionizing spectrum but, due to its radio-loudness, also because it presents a copious supply of relativistic, synchrotron-emitting particles, which can dramatically alter the excitation of the NLR (Aldrovandi \& Péquignot 1973; Ferland \& Mushotzky 1984; Gruenwald \& Viegas-Aldrovandi 1987). "Cosmic ray heating" boosts the strengths of low-ionization lines such as [N II] $\lambda \lambda 6548$, 6583 and [S II] $\lambda \lambda 6716,6731$ (Viegas-Aldrovandi \& Gruenwald 1990), which are normally underpredicted (e.g., Ho, Filippenko \& Sargent 1993; Lewis, Eracleous \& Sambruna 2003), and thereby help to constrain alternative solutions that invoke selective abundance enhancement of $\mathrm{N}$ and $\mathrm{S}$ (Storchi-Bergmann \& Pastoriza 1990) or dust grain depletion (Gabel et al. 2000). 


\subsection{Contribution from Fast Shocks}

Despite the natural appeal of AGN photoionization, alternative excitation mechanisms for LINERs have been advanced. Collisional ionization by shocks has been a popular contender from the outset (Burbidge, Gould \& Pottasch 1963; Osterbrock 1971; Osterbrock \& Dufour 1973; Koski \& Osterbrock 1976; Danziger, Fosbury \& Penston 1977; Fosbury et al. 1978; Ford \& Butcher 1979; Heckman 1980b; Baldwin, Phillips \& Terlevich 1981). Shocks continue to be invoked (Bonatto, Bica \& Alloin 1989; Dopita \& Sutherland 1995; Alonso-Herrero et al. 2000; Sugai \& Malkan 2000) even after concerns over the [O III] $\lambda 4363$ temperature problem had been dispelled as a result of either revised measurements (Keel \& Miller 1983; Rose \& Tripicco 1984) or complications arising from density stratification (Filippenko \& Halpern 1984). Dopita \& Sutherland (1995) showed that the diffuse radiation field generated by fast $\left(v \approx 150-500 \mathrm{~km} \mathrm{~s}^{-1}\right)$ shocks can reproduce the optical narrow emission lines seen in both LINERs and Seyferts. In their models, LINER-like spectra are realized under conditions in which the precursor H II region of the shock is absent, as might be the case in gas-poor environments. The postshock cooling zone attains a much higher equilibrium electron temperature than a photoionized plasma; consequently, a robust prediction of shock models is that shocked gas should produce a higher excitation spectrum, most readily discernible in the UV, than photoionized gas. In all the cases studied so far, however, the UV spectra are inconsistent with the fast-shock scenario because the observed intensities of high-excitation lines such as C IV $\lambda 1549$ and He II $\lambda 1640$ are much weaker than predicted (Barth et al. 1996, 1997; Maoz et al. 1998; Nicholson et al. 1998; Gabel et al. 2000). Dopita et al. (1997) used the spectrum of the circumnuclear disk of M87 to advance the view that LINERs are shock-excited. This argument is misleading because their analysis deliberately avoids the nucleus. Sabra et al. (2003) demonstrate that the UVoptical spectrum of the nucleus of M87 is best explained by a multi-component photoionization model.

Analysis of the emission-line profiles of the Palomar nuclei further casts doubt on the viability of the fast-shock scenario (Ho, Filippenko \& Sargent 2003). The velocity dispersions of the nuclear gas generally fall short of the values required for fast-shock excitation to be important. Furthermore, the close similarity between the velocity field of LINERs and Seyferts as deduced from their line profiles contradicts the basic premise that shocks are primarily responsible for the spectral differences between the two classes of objects. For a given bulge potential, LINERs, if anything, have smaller, not larger, gas velocity dispersions than Seyferts (L.C. Ho, in preparation). And as discussed in $\S 5.2$, the incidence of extended radio jets, the most likely source of kinetic energy injection into the NLR, is actually lower in LINERs than in Seyferts, again contrary to naive expectations.

Notwithstanding these complications, it is inconceivable that mechanical heating, especially by lower velocity $\left(\sim 50-100 \mathrm{~km} \mathrm{~s}^{-1}\right)$ shocks, does not play some role in the overall excitation budget of LINERs. The velocities of the lineemitting gas are, after all, highly supersonic, turbulent, and most likely pressuredominated (L.C. Ho, in preparation). The trick is to figure out what is the balance between shocks and photoionization, and what physical insights can be

gained from knowing the answer. It would be worthwhile to revisit composite shock plus photoionization models, such as those developed by Viegas-Aldrovandi \& Gruenwald (1990) and Contini (1997) with the latter component maximally 
constrained by observations so that robust, quantitative estimates can be placed on the former. Such an approach might yield meaningful measurements of the amount of mechanical energy deposited into the host galaxy by AGN feedback.

\subsection{Contribution from Stellar Photoionization}

Another widely discussed class of models invokes hot stars formed in a shortduration burst of star formation to supply the primary ionizing photons. Ordinary O-type stars with effective temperatures typical of those found in giant $\mathrm{H}$ II regions in galactic disks do not produce sufficiently strong low-ionization lines to account for the spectra of LINERs. The physical conditions in the centers of galaxies, on the other hand, may be more favorable for generating LINER-like spectra. For example, Terlevich \& Melnick (1985) postulate that the high-metallicity environment of galactic nuclei may be particularly conducive to forming very hot, $T \approx(1-2) \times 10^{5} \mathrm{~K}$, luminous Wolf-Rayet stars whose ionizing spectrum would effectively mimic the power-law continuum of an AGN. The models of Filippenko \& Terlevich (1992) and Shields (1992) appeal to less extreme conditions. These authors show that photoionization by ordinary O stars, albeit of somewhat higher effective temperature than normal (but see Schulz \& Fritsch 1994), embedded in an environment with high density and low ionization parameter can explain the spectral properties of transition objects. Barth \& Shields (2000) extended this work by modeling the ionizing source not as single O-type stars but as a more realistic evolving young star cluster. They confirm that young, massive stars can indeed generate optical emission-line spectra that match those of transition objects, and, under some plausible conditions, even those of bona fide LINERs. But there is an important caveat: the star cluster must be formed in an instantaneous burst, and its age must coincide with the brief phase $(\sim 3-5 \mathrm{Myr}$ after the burst) during which sufficient Wolf-Rayet stars are present to supply the extreme-UV photons necessary to boost the low-ionization lines. The necessity of a sizable population of Wolf-Rayet stars is also emphasized in the study by Gabel \& Bruhweiler (2002). As discussed in Ho, Filippenko \& Sargent (2003), the main difficulty with this scenario, and indeed with all models that appeal to young or intermediate-age stars (e.g., Engelbracht et al. 1998; Alonso-Herrero et al. 2000; Taniguchi, Shioya \& Murayama 2000), is that the nuclear stellar population of the host galaxies of the majority of nearby AGNs, irrespective of spectral class, is demonstrably old (§ 4.2). Stellar absorption indices indicative of young or intermediate-age stars are seldom seen, and the telltale emission features of Wolf-Rayet stars are notably absent, both in ground-based and HST spectra. Sarzi et al. (2005) find that young stars can account for at most a few percent of the blue light within the central few parsecs of nearby LLAGNs, in most cases incapable of providing enough ionizing photons to account for the observed $\mathrm{H} \alpha$ emission. Post-starburst scenarios face another serious dilemma: if most LLAGNs, which constitute the majority of nearby AGNs and a large percentage of all galaxies, are described by this scenario, then where are their precursors? They do not exist. These empirical facts seriously undermine the viability of starburst or post-starburst models for LLAGNs.

Evolved, low-mass stars, on the other hand, probably contribute at some level to the ionization. This idea was advocated by Binette et al. (1994), who proposed that post-asymptotic giant branch (post-AGB) stars, which can attain effective temperatures as high as $\sim 10^{5} \mathrm{~K}$, might be responsible for photoionizing the 
extended ionized gas often observed in elliptical galaxies. The emission-line spectrum of these nebulae, in fact, tend to be of relatively low ionization (DemoulinUlrich, Butcher \& Boksenberg 1984; Phillips et al. 1986). Invoking evolved stars has the obvious appeal of not violating the stellar population constraints discussed above. This mechanism, however, cannot be the dominant contributor to compact LINERs. The line emission tends to be very centrally concentrated (§ 6.1), much more so than the underlying stellar density profile. Moreover, the line strengths in most LLAGNs are simply too high. The calculations of Binette et al. predict $\mathrm{H} \alpha$ equivalent widths of $\mathrm{EW} \approx 1 \AA$, whereas the LINERs and transition objects in the Palomar survey have an average EW 3-4 times higher (Ho, Filippenko \& Sargent 2003), with over $70 \%$ of the sample having EW > 1 $\AA$.

To obtain a quantitative estimate of the contribution of post-AGB stars to the ionization budget of the weaker emission-line nuclei, I convert the nuclear stellar magnitudes $\left(m_{44}\right)$ given in Ho, Filippenko \& Sargent $(1997 a)$ to stellar masses assuming a mass-to-light ratio of $M / L_{B}=8\left(M / L_{B}\right)_{\odot}$ and that post-AGB stars have a specific ionization rate of $Q_{\text {ion }}=7.3 \times 10^{40}\left(M / M_{\odot}\right) \mathrm{s}^{-1}$ (Binette et al. 1994). Within the $100 \times 200 \mathrm{pc}$ aperture of the Palomar spectra, the integrated stellar mass is $\sim 10^{7}$ to $10^{10} M_{\odot}$, with a median value of $2 \times 10^{9} M_{\odot}$, which corresponds to an ionizing photon rate of $Q_{\text {ion }}=1.5 \times 10^{50} \mathrm{~s}^{-1}$. These estimates depend on the choice of the stellar initial mass function, and, most importantly, on detailed processes during the late stages of stellar evolution that are still not fully understood (see, e.g., O'Connell 1999). Nevertheless, taking the fiducial estimates as a rough guide, the predicted values of $Q_{\text {ion }}$ can be compared to the actually observed, extinction-corrected $\mathrm{H} \alpha$ luminosity. Assuming complete reprocessing of the ionizing continuum and that on average it takes 2.2 Lyman continuum photons to produce one $\mathrm{H} \alpha$ photon, I estimate that the nebular line emission in roughly one-third of the Palomar sources can be powered by photoionization from post-AGB stars. The fraction is higher for LINERs (39\%) than Seyferts (16\%), being most prevalent for LINER 2s (44\%) and transition objects (33\%). Eracleous, Hwang \& Flohic (2008b) performed a similar analysis for a handful of LINERs with central stellar luminosity profiles available from $H S T$, concluding also that post-AGB stars can alleviate the ionizing photon deficit in some objects.

\subsection{Energy Budget}

In luminous AGNs, important confirmation of the basic photoionization paradigm comes from the strong empirical scaling and correlated variability between line flux and the strength of the ionizing continuum. Although very little information exists in terms of line-continuum variability for LLAGNs, enough X-ray observations have now been amassed to examine the correlation between optical line luminosity and X-ray luminosity. The X-ray band is only indirectly coupled to the bulk of the Lyman continuum, but in LLAGNs, it offers the most reliable probe of the high-energy spectrum. Studies in the soft X-ray band suggest that LLAGNs roughly follow the extrapolation of the $L_{\mathrm{H} \alpha}-L_{\mathrm{X}}$ correlation established for higher luminosity sources (Koratkar et al. 1995; Roberts \& Warwick 2000; Halderson et al. 2001). Intriguingly, no clear differences could be discerned between LINERs and Seyferts, confirming preliminary evidence presented in Halpern \& Steiner (1983). A more complex picture, however, emerges at higher energies $(2-10 \mathrm{keV})$. Terashima, Ho \& Ptak (2000) and Terashima et al. (2000) 

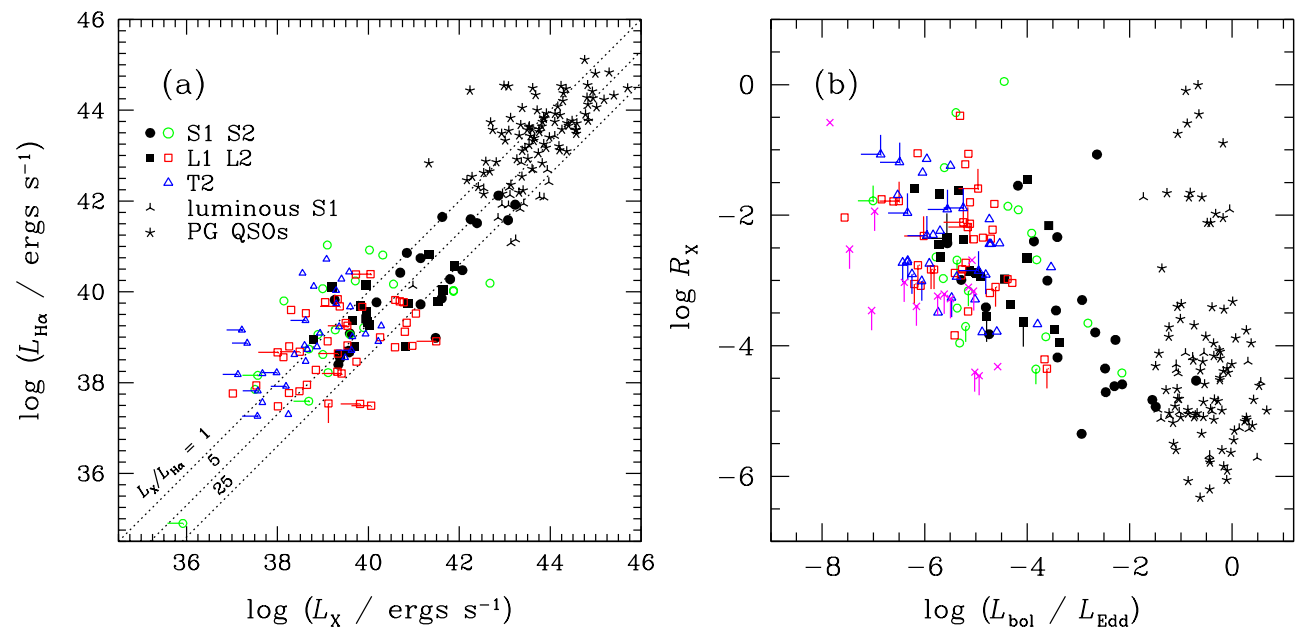

Figure 10: (a) Correlation between (total) $\mathrm{H} \alpha$ luminosity and X-ray $(2-10 \mathrm{keV})$ luminosity. The dotted lines mark $L_{\mathrm{X}} / L_{\mathrm{H} \alpha}=1,5$, and 25. (b) Distribution of radio-loudless parameter versus Eddington ratio, with the bolometric luminosity estimated assuming $L_{\mathrm{bol}}=16 L_{\mathrm{X}}$.

note that LINER $2 \mathrm{~s}$, unlike LINER 1s, suffer from a deficit of ionizing photons: the X-ray emission of the nucleus, when extrapolated to the UV, underpredicts the observed $\mathrm{H} \alpha$ luminosity by a factor of $\sim 10-100$. This trend persists in more recent Chandra observations (Terashima \& Wilson 2003b; Flohic et al. 2006; Eracleous, Hwang \& Flohic 2008b), one that seems to be especially endemic to transition objects (Ho et al. 2001; Filho et al. 2004).

Figure $10 a$ shows an update of the $L_{\mathrm{H} \alpha}-L_{\mathrm{X}}$ relation for all Palomar sources with high-resolution $\left(\lessgtr 5^{\prime \prime}\right) \mathrm{X}$-ray measurements. For comparison, I also include $z<0.5$ Palomar-Green (PG; Schmidt \& Green 1983) quasars and luminous Seyfert 1s with well-determined SEDs (Figure 7; L.C. Ho, in preparation). All broad-line sources follow an approximately linear relation over nearly 7 orders of magnitude in luminosity. In detail, $L_{\mathrm{H} \alpha} \propto L_{\mathrm{X}}^{1.1}$, such that quasars and luminous Seyferts have a median ratio $L_{\mathrm{X}} / L_{\mathrm{H} \alpha} \approx 2$, to be compared with $L_{\mathrm{X}} / L_{\mathrm{H} \alpha} \approx 7$ for Palomar Seyfert 1s. Ignoring the possible effect of a luminosity-dependent covering factor, this can be interpreted as the consequence of a decrease in the ratio of UV radiation to X-rays with decreasing luminosity, reflecting a pattern familiar from samples of bright AGNs (e.g., Strateva et al. 2005), now extended down to lower luminosities by $\sim 3$ orders of magnitude. Since LINER 1 s are weaker than Seyfert 1s and their SEDs lack a UV bump, it is surprising that they actually have a somewhat lower $L_{\mathrm{X}} / L_{\mathrm{H} \alpha}$ ratio $(\sim 5)$ than Seyfert $1 \mathrm{~s}$. This suggests that at least some of the $\mathrm{H} \alpha$ emission in LINER 1s, presumably in the narrow component, is not powered by AGN photoionization. In fact, this turns out to be a property symptomatic of all type 2 LLAGNs (Table 1). The most extreme manifestation can be seen among transition objects, with $L_{\mathrm{X}} / L_{\mathrm{H} \alpha} \approx 0.4$, but both LINER $2 \mathrm{~s}$ and Seyfert $2 \mathrm{~s}$ also exhibit an ionizing photon deficit. For conditions typical of LLAGNs, Eracleous, Hwang \& Flohic (2008b) use photoionization calculations to infer $L_{\mathrm{ion}}=18 L_{\mathrm{H} \alpha} f_{c}^{-1}$, where $L_{\mathrm{ion}}$ is the ionizing luminosity between 1 Ryd and $10 \mathrm{keV}$ and $f_{c}$ is the covering factor of the line-emitting gas. Since $L_{\mathrm{ion}} \approx 3 L_{\mathrm{X}}$ for a power-law spectrum with $\alpha=-0.1$ to $-0.9, L_{\mathrm{X}} / L_{\mathrm{H} \alpha} \approx 6 f_{c}^{-1}$. It is clear 
that most narrow-line LLAGNs violate this energy balance condition, even for the optimistic assumption of $f_{c}=1$.

There are several possible solutions to the ionization deficit problem. (1) The $\mathrm{X}$-rays could be highly obscured, perhaps even Compton-thick. In light of the evidence given in $\S \S 5.3,5.6$, I consider this solution to be untenable for LINER 2s; highly absorbed sources do exist (e.g., NGC 4261; Zezas et al. 2005), but they are in the minority. Moreover, many of the X-ray measurements have already been corrected for absorption. The situation is more complex for Seyfert 2s. Some of the sources with low $L_{\mathrm{X}} / L_{\mathrm{H} \alpha}$ indeed show direct evidence for Compton thickness from their X-ray spectra (Cappi et al. 2006). Others, however, are too faint for spectral analysis, and for these, their status as Compton-thick sources was based on the observed ratio of 2-10 keV flux to [O III] $\lambda 5007$ flux (Bassani et al. 1999; Panessa \& Bassani 2002). Applying an average correction factor of 60 to the X-ray luminosity would bring the Seyfert 2s into agreement with the Seyfert 1 s on the $L_{\mathrm{H} \alpha}-L_{\mathrm{X}}$ relation (Panessa et al. 2006). But this procedure assumes that the low values of $L_{\mathrm{X}} / L_{\mathrm{H} \alpha}$ are due to a reduction of the X-rays by absorption rather than an enhancement of $\mathrm{H} \alpha$ (see below). (2) The SED could be drastically different, specifically in having a much more prominent UV component. This proposition can be promptly dismissed because the SEDs of LLAGNs generically lack a UV bump. There is certainly no indication that type 2 sources are preferentially brighter in the UV; in the case of LINERs, type 2 sources, if anything, tend to be redder than type 1 sources (Maoz et al. 2005). (3) Lastly, and most plausibly, a significant fraction of the ionization for the narrow-line gas comes from nonnuclear sources. As discussed in connection with the preceding two subsections, young, massive stars and fast shocks are generally not viable options. There are a number of candidate sources of "extra" ionization, including hot, evolved stars, turbulent mixing layers, diffuse X-ray emitting plasma, low-mass XRBs, cosmic ray heating, and mechanical heating from radio jets. As all of these sources probably contribute at some level, efforts to single out any dominant mechanism may be hopelessly challenging. Nevertheless, as discussed in $\S 6.3$, post-AGB stars appear especially promising. Taking the calculations of Binette et al. (1994) as a guide, the stellar mass within the central $100-200$ pc region generates sufficient Lyman continuum photons to account for the $\mathrm{H} \alpha$ emission in $\sim 30 \%-40 \%$ of the LINER $2 \mathrm{~s}$ and transition objects. This estimate is crude and admittedly optimistic, as it assumes a covering factor of unity for the NLR, but it serves as a useful illustration of the types of effects that should be included in any complete treatment of the energy budget problem in LLAGNs.

\subsection{The Nature of Transition Objects and a Unified View of LLAGNs}

The physical origin of transition nuclei continues to be a thorny, unresolved problem. In standard line-ratio diagrams (Figure 3), transition nuclei are empirically defined to be those sources that lie sandwiched between the loci of "normal" H II regions and LINERs. This motivated Ho, Filippenko \& Sargent (1993) to propose that transition objects may be composite systems consisting of a LINER nucleus plus an H II region component. The latter could arise from neighboring circumnuclear H II regions or from H II regions randomly projected along the line of sight. A similar argument, based on decomposition of line profiles, was made by Véron, Gonçalves \& Véron-Cetty (1997). If transition objects truly are 
LINERs sprinkled with a frosting of star formation, one would expect that their host galaxies should be similar to those of LINERs, modulo minor differences due to extra contaminating star formation. The study of Ho, Filippenko \& Sargent (2003) tentatively supports this picture. The host galaxies of transition nuclei exhibit systematically higher levels of recent star formation, as indicated by their far-IR emission and broad-band optical colors, compared to LINERs of matched morphological types. Moreover, the hosts of transition nuclei tend to be slightly more inclined than LINERs. Thus, all else being equal, transition-type spectra seem to be found precisely in those galaxies whose nuclei have a higher probability of being contaminated by extra-nuclear emission from star-forming regions.

This story, however, has some holes. If spatial blending of circumnuclear H II regions is sufficient to transform a regular LINER into a transition object, the LINER nucleus should reveal itself unambiguously in spectra taken with angular resolution sufficiently high to isolate it. This test was performed by Barth, Ho \& Filippenko (2003), who obtained HST/STIS spectra, taken with a $0{ }^{\prime \prime} 2$-wide slit, of a well-defined subsample of 15 transition objects selected from the Palomar catalog. To their surprise, the small-aperture spectra of the nuclei, for the most part, look very similar to the ground-based spectra; they are not more LINERlike. Shields et al. (2007) reached the same conclusion from their STIS study of Palomar nuclei, which included six transition objects, showing that even at HST resolution these objects do not reveal the expected excitation gradients.

The "masqueraded-LINER" hypothesis can be further tested by searching for compact radio and X-ray cores using high-resolution images. Recall that this is a highly effective method to filter out weak AGNs ( $\S 5.2,5.3)$. Filho, Barthel \& Ho (2000, 2002a; Filho et al. 2004) have systematically surveyed the full sample of Palomar transition objects using the VLA at $8.4 \mathrm{GHz}$. They find that $\sim 25 \%$ of the population contains arcsecond-scale radio cores. These cores appear to be largely nonstellar in nature. The brighter subset of these sources that are amenable to follow-up Very Long Baseline Interferometry (VLBI) observations (Filho et al. 2004) all reveal more compact (milliarcsecond-scale) cores with flat radio spectra and high brightness temperatures $\left(z 10^{7} \mathrm{~K}\right)$. These radio statistics are hard to interpret, however, in the absence of a control sample of other LLAGNs surveyed to the same depth, resolution, and wavelength. The Nagar, Falcke \& Wilson (2005) $15 \mathrm{GHz}$ survey satisfies these criteria. As Table 1 shows, the frequency of radio cores in transition objects is roughly half of that in Seyfert $2 \mathrm{~s}$ and LINER $2 \mathrm{~s}$. On the other hand, the detection rate of X-ray cores is actually remarkably high-74\% - identical to that of LINER 2 s and similar to that of Seyfert $2 \mathrm{~s}$. This observation strongly suggests that the majority of transition objects indeed do harbor AGNs.

In light of these recent developments, the basic picture for the physical nature of transition objects needs to be revised. Inspection of the statistical properties in Table 1 offers the following clues, which help not only to explain transition objects but provide a unified view to relate the different classes of LLAGNs.

1. Seyferts, LINERs, and transition objects define a sequence of decreasing accretion rate. This is most evident from $L_{\mathrm{X}}$ and $L_{\mathrm{bol}} / L_{\mathrm{Edd}}$, but it is also seen in $L_{\mathrm{H} \alpha}$ and $P_{\mathrm{rad}}$.

2. As noted in $\S 5.10$, type 1 sources have significantly higher luminosities and Eddington ratios than type 2 systems. The basic premise of the conventional orientation-based unification scenario does not hold for LLAGNs. 
The systematic reduction in accretion rate along the sequence $\mathrm{S} \rightarrow \mathrm{L} \rightarrow \mathrm{T}$ also provides a viable explanation for the systematic decrease in the detection rate of broad $\mathrm{H} \alpha$ emission, especially the precipitous drop among transition sources $\left(f_{b}\right.$ in Table 1$)$.

3. Transition objects appear to be anomalously strong in their $\mathrm{H} \alpha$ emission. In light of the $H S T$ evidence for a distributed source of ionization, I suspect that a significant fraction of the $\mathrm{H} \alpha$ emission in these objects in fact is not photoionized by the central AGN. This leads to misleading values of $L_{\mathrm{X}} / L_{\mathrm{H} \alpha}$ and $R_{o}$ (which is based on $L_{\mathrm{H} \alpha}$ ). For this class either the X-ray or radio core provides a cleaner measure of the AGN power.

4. The loose inverse correlation between radio-loudness and accretion rate, best seen by comparing $R_{\mathrm{X}}$ versus either $L_{\mathrm{X}}$ or $L_{\mathrm{X}} / L_{\mathrm{Edd}}$, mirrors the trends found by Ho (2002a) and Terashima \& Wilson (2003b).

5. Focusing on the type 2 sources, note that LINER $2 \mathrm{~s}$ are very similar to Seyfert $2 \mathrm{~s}$, the former being $\sim 1 / 3-1 / 2$ as strong as the latter in terms of $\mathrm{H} \alpha$ luminosity and radio power. The two groups have almost identical $L_{\mathrm{X}}$ and $L_{\mathrm{X}} / L_{\mathrm{Edd}}$, although this may be an artifact of incomplete absorption correction for Seyfert 2s, some of which are highly absorbed (Panessa et al. 2006). In the same vein, I propose that transition objects represent the next step in the luminosity sequence. Judging by their X-ray luminosity, radio power, $L_{\mathrm{X}} / L_{\mathrm{Edd}}$, and radio detection fraction, the AGN component in transition objects is $\sim 1 / 4$ to $1 / 2$ as strong as that in LINER $2 \mathrm{~s}$.

According to the picture just outlined, most, if not all, type 2 sources are genuinely accretion powered. Using the accretion rate as the metric for the level of AGN activity, Seyfert 1s rank at the top of the scale, followed by Seyfert 2s, LINER 1s, LINER 2s, and finally ending with transition objects. This scenario, which in broad-brush terms explains a wide range of data summarized in Table 1, has the virtue of simplicity. It is also physically appealing, given the broad spectrum of accretion rates anticipated in nearby galaxies.

There is, however, one loose end that needs to be tied. What powers the spatially extended, "excess" optical line emission in transition objects? For the reasons explained before, the source of the ionization is unlikely to be shock heating or photoionization by hot, massive stars, notwithstanding the success with which such models have been applied to some individual objects (Engelbracht et al. 1998; Barth \& Shields 2000; Gabel \& Bruhweiler 2002). Shields et al. (2007) suggest two candidates for a spatially extended source of ionization: hot, evolved stars and turbulent mixing layers in the interstellar medium (Begelman \& Fabian 1990). In $\S 6.3$, I showed that the stellar mass in the central 100-200 pc indeed seems to provide enough post-AGB stars to account for the correct level of $\mathrm{H} \alpha$ emission in a significant fraction of the transition objects. I would like to suggest two other sources, ones that have the advantage of being empirically well motivated by recent observations. These processes probably operate in all nuclear environments all the time, maintaining a "baseline" level of weak optical line emission that is only noticeable after the AGN has subsided to a very low level.

As discussed in $\S \S 5.3,5.4$, the X-ray morphology of the central few hundred parsecs of galaxies can be quite complex. The nucleus, if present, is often encircled by other point sources, mostly XRBs (Fabbiano 2006). With X-ray luminosities ranging from $\sim 10^{37}$ to $10^{39} \mathrm{ergs} \mathrm{s}^{-1}$ (Flohic et al. 2006), XRBs individually or 
collectively can outshine the nucleus itself (Ho et al. 2001; Eracleous et al. 2002; Ho, Terashima \& Ulvestad 2003; see Figure 5). The discrete sources themselves are embedded in extended emission, consisting of an optically thin thermal plasma with $k T \approx 0.5 \mathrm{keV}$ and a spectrally harder diffuse component, which contributes a luminosity of $\sim(5-9) \times 10^{38} \mathrm{ergs} \mathrm{s}^{-1}$ in the $0.5-10 \mathrm{keV}$ band (Flohic et al. 2006). The hard diffuse component most likely represents the cumulative emission from faint, unresolved low-mass XRBs, although this interpretation seems somewhat at odds with the spectrum derived by Flohic et al. (power law with $\alpha=-0.3$ to $-0.5)$. Low-mass XRBs typically can be fit by a thermal bremsstrahlung model with $k T=5-10 \mathrm{keV}$ or a power law with $\alpha=-0.6$ to -0.9 (e.g., Makishima et al. 1989). High-mass XRBs would provide a better match to the observed spectrum, but in view of what we know about the stellar populations ( $\S 4.2$ ), they are probably untenable. A possible solution is to invoke a multi-temperature plasma (M. Eracleous, private communications); a hotter component $(k T \approx$ few $\mathrm{keV})$, when added to the cooler $k T=0.5 \mathrm{keV}$ component, would presumably permit a significant contribution from low-mass XRBs without violating the spectral constraints. We can estimate the expected X-ray output from low-mass XRBs from the correlation between optical and X-ray luminosity established for normal galaxies (Fabbiano \& Trinchieri 1985). Using again the nuclear stellar magnitudes from the Palomar survey, I obtain a median $L_{\mathrm{X}}(2-10 \mathrm{keV})=(3 \pm 1) \times 10^{38}$ ergs $\mathrm{s}^{-1}$ within the central $2^{\prime \prime} \times 4^{\prime \prime}$ aperture, which falls within the ballpark of the value measured by Flohic et al. (2006). The combination of hot gas and $\mathrm{XRB}$ emission, therefore, supplies $\sim 10^{39} \mathrm{ergs} \mathrm{s}^{-1}$ in X-rays, comparable to the amount coming from the nucleus alone for Seyfert 2s and LINER 2s, and double the amount from transition nuclei (Table 1). Voit \& Donahue (1997) suggest that hot plasma additionally may transfer heat conductively to the line-emitting gas in LINERs, in a manner analogous to the situation in cooling flow filaments in galaxy clusters.

Lastly, cosmic ray heating ( $\S 6.1$ ) by the central radio core will further enhance the optical line luminosity (Ferland \& Mushotzky 1984). The very source of the fast particles, namely compact radio jets, itself probably injects an additional source of mechanical heating, although this is more difficult to model concretely. Both processes-photoionization by off-nuclear X-rays and cosmic ray heating - have a convenient virtue: they will tend to produce low-ionization spectra and therefore provide a natural match to the spectral characteristics of nearby LLAGNs.

\section{IMPLICATIONS FOR BLACK HOLE DEMOGRAPHICS}

As summarized by Kormendy (2004), spatially resolved kinematical observations have convincingly measured $\mathrm{BH}$ masses in a sizable number of nearby galaxies, to the point that important inferences on their demographics can be drawn (Richstone 2004). Following an argument first due to Soltan (1982), comparison of the integrated radiation density from quasars to the integrated mass density in local BHs shows that BHs have grown mostly via a radiatively efficient mode of accretion during their bright AGN phase (e.g., Yu \& Tremaine 2002). Nearby galaxies, therefore, should be home to AGN relics.

The LLAGNs summarized in this review provide an important confirmation of this basic prediction. Not only are weakly accreting BHs found in great abun- 

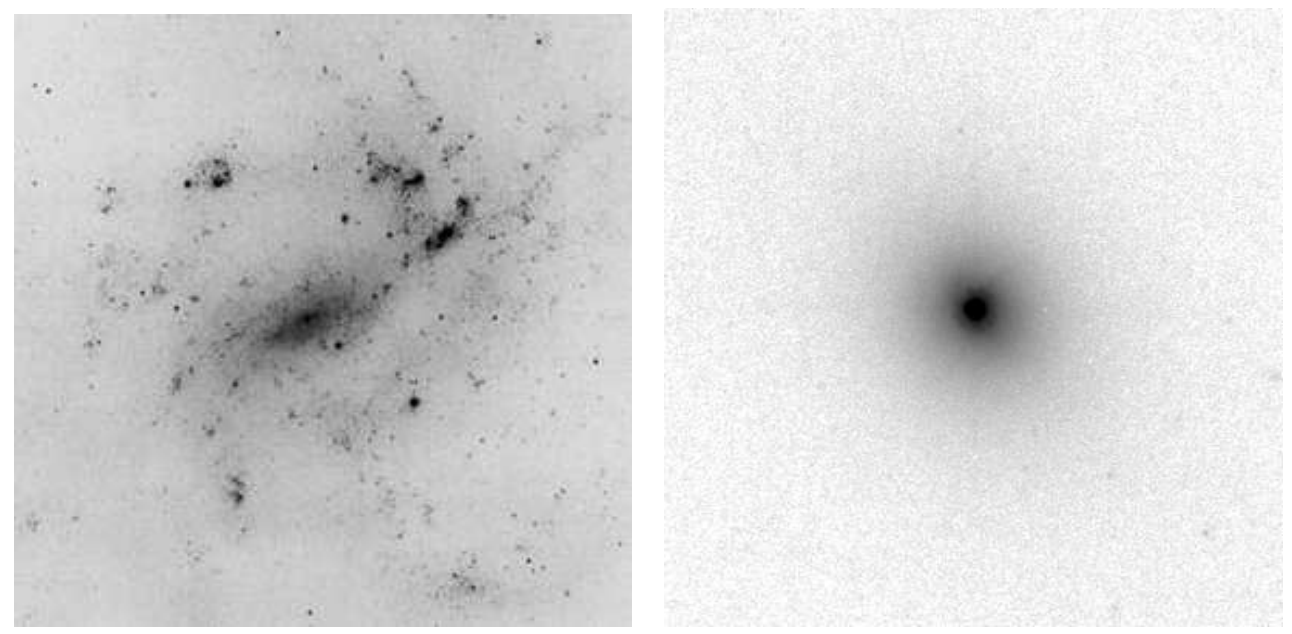

Figure 11: Two examples of AGNs in late-type galaxies. The left panel shows an optical image of NGC 4395, adapted from the Carnegie Atlas of Galaxies (Sandage \& Bedke 1994); the image is $\sim 15^{\prime}(17 \mathrm{kpc})$ on a side. The right panel shows an HST I-band image of POX 52, adapted from C.E. Thornton, A.J. Barth, L.C. Ho, R.E. Rutledge, J.E. Greene (in preparation); the image is $\sim 11^{\prime \prime}$ (5 kpc) on a side.

dance in the local Universe, but they are found where prevailing wisdom says that they should be found, namely in the centers of galaxies that contain bulges. Among E, S0, and Sb galaxies, the AGN detection rate is $\sim 50 \%$, increasing to over $70 \%$ among Sa galaxies. Since sensitivity and confusion impact the detection rates, these statistics are not inconsistent with the notion that BHs are ubiquitous in essentially all E-Sb galaxies.

Notably, the incidence of AGNs plummets for galaxies with Hubble types Sc and later, precisely at the point where classical $\left(r^{1 / 4}\right.$ profile) bulges effectively cease to exist and secular dissipation processes begin to kick in (Kormendy \& Kennicutt 2004). Although the AGN fraction is low for late-type spirals, it is also not zero. Careful scrutiny of this minority population addresses two important questions: (1) Are there central (nonstellar) BHs with masses below $10^{6} M_{\odot}$ ? (2) Must central BHs always be encased in a bulge?

Two remarkable galaxies give the clearest testimony that low-mass $\mathrm{BHs}$ do, in fact, exist. Within the Palomar survey, the nearby ( $\sim 4 \mathrm{Mpc}$ ) galaxy NGC 4395 contains all the usual attributes of a self-respecting AGN: broad optical and UV emission lines (Filippenko \& Sargent 1989; Filippenko, Ho \& Sargent 1993), a compact radio core (Ho \& Ulvestad 2001) of high brightness temperature (Wrobel, Fassnacht \& Ho 2001; Wrobel \& Ho 2006), and rapidly variable hard X-ray emission (Shih, Iwasawa \& Fabian 2003; Moran et al. 2005). Contrary to expectations, however, NGC 4395 is an extremely late-type (Sdm) spiral (Figure 11, left), whose central stellar velocity dispersion does not exceed $\sim 30 \mathrm{~km} \mathrm{~s}^{-1}$ (Filippenko \& Ho 2003). If NGC 4395 obeys the $M_{\mathrm{BH}}-\sigma$ relation, its central BH should have a mass $\lesssim 10^{5} M_{\odot}$. This limit agrees surprisingly well with the value of $M_{\mathrm{BH}}$ estimated from its broad $\mathrm{H} \beta$ line width or X-ray variability properties $\left(\sim 10^{4}-10^{5} M_{\odot} ;\right.$ Filippenko \& Ho 2003$)$, or from reverberation mapping $\left(3.6 \times 10^{5}\right.$ $M_{\odot}$; Peterson et al. 2005). POX 52 (Figure 11, right) presents another interesting case. As first noted by Kunth, Sargent \& Bothun (1987), the presence of a 
Seyfert-like nucleus in POX 52 is unusual because of the low luminosity of the host galaxy. Barth et al. (2004) show that POX 52 bears a close spectroscopic resemblance to NGC 4395. Based on the broad profile of $\mathrm{H} \beta$, these authors derive a virial $\mathrm{BH}$ mass of $1.6 \times 10^{5} M_{\odot}$ for POX 52 , again surprisingly close to the value of $1.3 \times 10^{5} M_{\odot}$ predicted from the $M_{\mathrm{BH}}-\sigma$ relation given the measured central stellar velocity dispersion of $35 \mathrm{~km} \mathrm{~s}^{-1}$.

The two objects highlighted above demonstrate that the mass spectrum of nuclear BHs indeed does indeed extend below $10^{6} M_{\odot}$, providing great leverage for anchoring the $M_{\mathrm{BH}}-\sigma$ at the low end. Furthermore, they shed light on the variety of environments in which nuclear BHs can form, providing muchneeded empirical clues to the conditions that fostered the formation of the seeds for supermassive BHs. NGC 4395 has little or no bulge, but it does have a compact, central star cluster in which the BH is embedded (Filippenko \& Ho 2003). Interestingly, G1, a massive star cluster in M31 that to date contains the best direct detection of an intermediate-mass $\left(\sim 20,000 M_{\odot}\right) \mathrm{BH}$ (Gebhardt, Rich \& Ho 2002, 2005; Ulvestad, Greene \& Ho 2007), is thought to be the stripped nucleus of a once small galaxy. The same holds for the Galactic cluster $\omega$ Cen, for which Noyola, Gebhardt \& Bergmann (2008) report a central dark mass of $5 \times 10^{4} M_{\odot}$. POX 52 is equally striking. Deep images reveal POX 52 to be most akin to a spheroidal galaxy (Barth et al. 2004; C.E. Thornton, A.J. Barth, L.C. Ho, R.E. Rutledge, J.E. Greene, in preparation), to date an unprecedented morphology for an AGN host galaxy. This is quite unexpected because spheroidal galaxies, while technically hot stellar systems, bear little physical resemblance to classical bulges. Spheroidals occupy a distinct locus on the fundamental plane (e.g., Geha, Guhathakurta \& van der Marel 2002; Kormendy et al. 2008), and they may originate from harassment and tidal stripping of late-type disk galaxies (e.g., Moore et al. 1996). Thus, like NGC 4395, POX 52 stands as testimony that a classical bulge is not a prerequisite for the formation of central $\mathrm{BH}$.

But how common are such objects? AGNs hosted in high-surface brightness, late-type spirals appear to be quite rare in the nearby Universe. Within the comprehensive Palomar survey, NGC 4395 emerges as a unique case of an unambiguous broad-line AGN hosted in a late-type system. The majority of late-type spirals do possess compact, photometrically distinct nuclei (Böker et al. 2002), morphologically not dissimilar from that in NGC 4395, but, with few exceptions (Shields et al. 2008), these nuclei are compact star clusters with no compelling evidence for an accompanying accreting central BH (Walcher et al. 2006). Nuclear star clusters do not appear to directly impact a galaxy's ability to host an AGN (Seth et al. 2008). Several serendipitous cases of AGNs in late-type galaxies have recently been found from analysis of Spitzer mid-IR spectra (Satyapal et al. 2007, 2008), as well as a number of AGN candidates from inspection of Chandra images (Desroches \& Ho 2008). Among earlier Hubble types, Gallo et al. (2008) report the detection of X-ray nuclei in two low-luminosity early-type galaxies.

To assess the true incidence of AGNs like NGC 4395 and POX 52 requires a spectroscopic survey much larger than Palomar. Greene \& Ho (2007b) performed a systematic spectral analysis of over 500,000 SDSS spectra with $z<0.35$ to search for broad-line AGNs, producing not only a detailed BH mass function for low-redshift AGNs (Greene \& Ho 2007a) but also a comprehensive catalog of $\sim 200$ low-mass $\left(M_{\mathrm{BH}}<10^{6} M_{\odot}\right)$ objects. Not much is known yet about the host galaxies, except that on average they are about 1 mag fainter than $L^{*}$. HST imaging of the initial sample of 19 objects discovered by Greene \& Ho (2004) 
reveal that the host galaxies are either mid- to late-type spirals (although none seems as late-type as NGC 4395) or compact, spheroidal-looking systems not unlike POX 52 (Greene, Ho \& Barth 2008). When projected onto the galaxy fundamental plane, the "bulge" component in some systems resides within the locus of spheroidal galaxies. Follow-up high-dispersion spectroscopy shows that these objects approximately follow the $M_{\mathrm{BH}}-\sigma$ relation (Barth, Greene \& Ho 2005).

\section{IMPLICATIONS FOR ACCRETION PHYSICS}

\subsection{Why Are LLAGNs So Dim?}

This review highlights a class of galactic nuclei that are extraordinary for being so ordinary. At their most extreme manifestation, LLAGNs emit a billion times less light than the most powerful known quasars. When quasars were first discovered, the challenge then was to explain their tremendous luminosities. Ironically, more than four decades later, the problem has been reversed: the challenge now is to explain how dead quasars can remain so dormant. The luminosity deficit problem was noted by Fabian \& Canizares (1988), who drew attention to the fact that elliptical galaxies, despite being suffused with a ready supply of hot gas capable of undergoing spherical accretion, have very dim nuclei. We can no longer speculate that these systems lack supermassive BHs, for we now know that they are there, at least in galaxies with bulges. And as I have shown in this review, the problem is by no means confined to ellipticals either.

Explanations of the luminosity paradox fall in several categories.

- Obscuration This trivial possibility can be summarily dismissed as a general solution in light of the evidence presented in $\S \S 5.3,5.6$.

- Low accretion rate A more plausible strategy is to starve the BH. Presentday massive galaxies, after all, should be relatively gas-poor, especially in their central regions, which are largely devoid of significant ongoing star formation. This argument quickly falls apart when one realizes just how little material is needed to light up the nuclei. The bolometric luminosities of nearby nuclei span $\sim 10^{38}-10^{44} \mathrm{ergs} \mathrm{s}^{-1}$, with a median value of $L_{\mathrm{bol}}=3 \times$ $10^{40} \mathrm{ergs} \mathrm{s}^{-1}$ and half of the sample lying between $3 \times 10^{39}$ and $3 \times 10^{41} \mathrm{ergs}$ $\mathrm{s}^{-1}$. For a canonical radiative efficiency of $\eta=0.1$, the required accretion rate is merely $\dot{M}=L_{\text {bol }} / \eta c^{2}=5 \times 10^{-6 \pm 1} M_{\odot} \mathrm{yr}^{-1}$. This is a pitifully miniscule amount, in comparison with the amount of fuel actually available to be accreted. Galactic nuclei unavoidably receive fuel from two sources: ordinary mass loss from evolved stars and gravitational capture of gas from the hot interstellar medium.

During the normal course of stellar evolution, evolved stars return a significant fraction of their mass to the ISM through mass loss. For a Salpeter stellar initial mass function with a lower-mass cutoff of $0.1 M_{\odot}$, an uppermass cutoff of $100 M_{\odot}$, solar metallicities, and an age of 15 Gyr, Padovani \& Matteucci (1993) estimate that

$$
\dot{M}_{*} \approx 3 \times 10^{-11}\left(\frac{L}{L_{\odot, V}}\right) \quad M_{\odot} \mathrm{yr}^{-1} .
$$

This result is consistent, within a factor of $\sim 2$, with the work of Ciotti et al. 
(1991) and Jungwiert, Combes \& Palous (2001). HST images reveal that galaxies contain central density concentrations, either in the form of nuclear cusps or photometrically distinct, compact stellar nuclei. The cusp profiles continue to rise to the resolution limit of $\operatorname{HST}\left(0 .{ }^{\prime \prime} 1\right)$, which is $r \approx 10 \mathrm{pc}$ at a distance of $20 \mathrm{Mpc}$, where $\rho \approx 10-10^{3} L_{\odot, V} \mathrm{pc}^{-3}$ for the "core" ellipticals and $\rho \approx 10^{2}-10^{4} L_{\odot, V} \mathrm{pc}^{-3}$ for the "power-law" ellipticals and bulges of early-type spirals and S0s (e.g., Faber et al. 1997). Within a spherical region of $r=10 \mathrm{pc}$, the diffuse cores have $L \approx 4 \times 10^{4}-4 \times 10^{6} L_{\odot, V}$, which yields $\dot{M}_{*} \approx 1 \times 10^{-6}-1 \times 10^{-4} M_{\odot} \mathrm{yr}^{-1}$; for the denser power-law cusps, $L \approx 4 \times 10^{5}-4 \times 10^{7} L_{\odot, V}$, or $\dot{M}_{*} \approx 1 \times 10^{-5}-1 \times 10^{-3} M_{\odot} \mathrm{yr}^{-1}$. Centrally dominant nuclear star clusters, present in a large fraction of disk galaxies, typically have luminosities $L \approx 10^{7} L_{\odot}$ (Carollo et al. 1997; Böker et al. 2002), and hence $\dot{M}_{*} \approx 10^{-3} M_{\odot} \mathrm{yr}^{-1}$.

Diffuse, hot gas in the central regions of galaxies holds another potential fuel reservoir. Low-angular momentum gas sufficiently close to a $\mathrm{BH}$ can accrete spherically in the manner described by Bondi (1952). To estimate the Bondi accretion rate, one needs to know the gas density and temperature at the accretion radius, $R_{a} \approx G M_{\mathrm{BH}} / c_{s}^{2}$, where $c_{s} \approx 0.1 T^{1 / 2} \mathrm{~km} \mathrm{~s}^{-1}$ is the sound speed of the gas at temperature $T$. The mass accretion rate follows from the continuity equation, $\dot{M}_{\mathrm{B}}=4 \pi R_{a}^{2} \rho_{a} c_{s}$, where $\rho_{a}$ is the gas density at $R_{a}$. Expressed in terms of typical observed parameters (see below),

$$
\dot{M}_{\mathrm{B}} \approx 7.3 \times 10^{-4}\left(\frac{M_{\mathrm{BH}}}{10^{8} M_{\odot}}\right)^{2}\left(\frac{n}{0.1 \mathrm{~cm}^{-3}}\right)\left(\frac{200 \mathrm{~km} \mathrm{~s}^{-1}}{c_{s}}\right)^{3} M_{\odot} \mathrm{yr}^{-1} .
$$

Chandra observations with sufficient resolution to resolve $R_{a}$ find that the diffuse gas in the central regions of elliptical galaxies typically has temperatures of $k T \approx 0.3-1 \mathrm{keV}$ and densities of $n \approx 0.1-0.5 \mathrm{~cm}^{-3}$ (e.g., Di Matteo et al. 2001, 2003; Loewenstein et al. 2001; Pellegrini 2005). Our knowledge of the hot gas content in the central regions of the bulges of spiral and S0 galaxies is more fragmentary. Chandra has so far resolved the hot gas in the centers of a handful of bulges (Milky Way: Baganoff et al. 2003; M31: Garcia et al. 2005; M81: Swartz et al. 2002; NGC 1291: Irwin, Sarazin \& Bregman 2002; NGC 1553: Blanton, Sarazin \& Irwin 2001; Sombrero: Pellegrini et al. 2003a). These studies suggest that bulges typically have gas temperatures of $k T \approx 0.3-0.6 \mathrm{keV}$. Information on gas densities is sketchier, but judging from the work on M81 and the Sombrero, a fiducial value might be $n \approx 0.1 \mathrm{~cm}^{-3}$.

If, for simplicity, we assume that the hot gas in most bulges is characterized by $n=0.1 \mathrm{~cm}^{-3}$ and $k T=0.3 \mathrm{keV}$, then $\dot{M}_{\mathrm{B}} \approx 10^{-5}-10^{-3} M_{\odot} \mathrm{yr}^{-1}$ for $M_{\mathrm{BH}}=10^{7}-10^{8} M_{\odot}$. In elliptical galaxies $M_{\mathrm{BH}} \approx 10^{8}-10^{9} M_{\odot}$, and for $n=0.2 \mathrm{~cm}^{-3}$ and $k T=0.5 \mathrm{keV}, \dot{M}_{\mathrm{B}} \approx 10^{-4}-10^{-2} M_{\odot} \mathrm{yr}^{-1}$. We note that these estimates of the Bondi accretion rates, which fall within the range given in recent compilations (e.g., Donato, Sambruna \& Gliozzi 2004; Pellegrini 2005; Soria et al. 2006), are probably lower limits because the actual densities near $R_{a}$ are likely to be higher than we assumed. For the above fiducial temperatures and $\mathrm{BH}$ masses, $R_{a} \approx 1-10 \mathrm{pc}$ for bulges and $\sim 10-100 \mathrm{pc}$ for ellipticals, roughly an order of magnitude smaller than the typical linear resolution achieved by Chandra for nearby galaxies. In well-resolved cases, the gas temperature profile generally remains constant 
to within $\sim 50 \%$ whereas the density typically increases by a factor of a few toward the center (e.g., Milky Way: Baganoff et al. 2003; M31: Garcia et al. 2005; M87: Di Matteo et al. 2003; NGC 1316: Kim \& Fabbiano 2003). Although the above estimates are very rough, and they are valid only in a statistical sense, one cannot escape the conclusion that in general in the central few parsecs of nearby galaxies $\dot{M}_{\mathrm{B}}+\dot{M}_{*} \gg \dot{M}$. Although meager, the joint contributions from stellar mass loss and Bondi accretion, if converted to radiation with $\eta=0.1$, would generate nuclei far more luminous than actually observed. The net accretion from the Bondi flow would be considerably reduced if the gas possesses some angular momentum at large radii (Proga \& Begelman 2003) — as inevitably it must - but even so it seems likely that the $\mathrm{BH}$ still has plenty of food at its disposal. LLAGNs are by no means fuel-starved. Moreover, the above estimates have erred on the conservative side. For example, I have assumed that all of the stars are evolved, although in reality most nuclei have composite populations and hence larger mass loss rates. Furthermore, I have neglected additional dissipation from larger scales (e.g., due to nuclear bars or spirals), as well as discrete, episodic events such as stellar tidal disruptions, which can provide a significant source of fuel, especially for BHs with masses $\lesssim 10^{7} M_{\odot}$ (Milosavljević, Merritt \& Ho 2006). All of these additional sources will only exacerbate the fuel surplus crisis.

- Low radiative efficiency If accretion does proceed at a reasonable fraction of the supply rate, then one has no option but to conclude that the radiative efficiency is much less than $\eta=0.1$, the standard value for an optically thick, geometrically thin disk. This type of argument has been invoked to explain the apparent conflict between the nuclear luminosities and Bondi accretion rates in many early-type galaxies (e.g., Fabian \& Rees 1995; Reynolds et al. 1996; Di Matteo \& Fabian 1997; Mahadevan 1997; Di Matteo et al. 2001, 2003; Loewenstein et al. 2001; Ho, Terashima \& Ulvestad 2003; Pellegrini et al. 2003a; Donato, Sambruna \& Gliozzi 2004; Evans et al. 2006). Accretion flows with low radiative efficiency, of which the most popular version is the ADAF (see reviews in Narayan 2002; Yuan 2007), arise when the accreting medium is sufficiently tenuous that its cooling time exceeds the accretion timescale. RIAFs are predicted to exist for accretion rates below a critical threshold of $\dot{M}_{\text {crit }} \approx 0.3 \alpha^{2} \dot{M}_{\text {Edd }} \approx 0.01 \dot{M}_{\text {Edd }}$, where the Shakura \& Sunyaev (1973) viscosity parameter is taken to be $\alpha \approx 0.1-0.3$ and $\dot{M}_{\mathrm{Edd}} \equiv L_{\mathrm{Edd}} / \eta c^{2}=0.22(\eta / 0.1)\left(M_{\mathrm{BH}} / 10^{8} M_{\odot}\right) M_{\odot} \mathrm{yr}^{-1}$. LLAGNs lie comfortably below this threshold.

- Inefficient accretion/jet feedback Precisely how low $\eta$ can become depends on how much of the native fuel supply actually gets accreted. In the presence of some rotation in the ambient medium, numerical simulations find that the amount of material accreted is much less than is available at large radii (e.g., Stone \& Pringle 2001; Igumenshchev, Narayan \& Abramowicz 2003). Since the gravitational binding energy in a RIAF cannot be radiated efficiently, it must be lost by nonradiative means (Blandford \& Begelman 1999), either through convective transport of energy and angular momentum to large radii or through a global outflowing wind (see review by Quataert 2003). The net effect of either process is to flatten the density profile near the center and to dramatically reduce the accretion rate. At very low accretion rates 
$\lesssim\left(10^{-5}-10^{-6}\right) \dot{M}_{\text {Edd }}$, such as in Sgr A* and some giant elliptical galaxies, electron heat conduction will further suppress the accretion rate (Johnson \& Quataert 2007). While these effects will ease the burden of invoking extremely small and perhaps physically unrealistic radiative efficiencies, it is important to note that these more recent models are still radiatively inefficient.

Whether the outflows generated in RIAFs can lead directly to relativistic jets is unclear, but what observations have established is the tendency for lowly accreting systems to become increasingly jet-dominated. We see this not only in FR I radio galaxies (Chiaberge, Capetti \& Celotti 1999; Donato, Sambruna \& Gliozzi 2004; Kharb \& Shastri 2004; Chiaberge, Capetti \& Macchetto 2005; Balmaverde \& Capetti 2006; Wu, Yuan \& Cao 2007), but it is also reflected in the nuclear properties of more run-of-the-mill LLAGNs ( $\S \S 5.3,5.8$ ), as well as in nearly quiescent nuclei (Pellegrini et al. 2007; Wrobel, Terashima \& Ho 2008). Detailed analysis of some sources, in fact, indicates that most of the accretion power is not radiated but instead channeled into the kinetic energy of relativistic jets (M87: Di Matteo et al. 2003; IC 1459: Fabbiano et al. 2003; IC 4296: Pellegrini et al. 2003b). By analogy with the situation in cooling flows in galaxy clusters, the propensity for LLAGNs to become radio-loud opens up the possibility that the kinetic energy from small-scale jets or collimated outflows provides a major source of "feedback" into the circumnuclear environment, perhaps to the extent that accretion can be significantly interrupted or curtailed (Binney \& Tabor 1995; Di Matteo et al. 2003; Pellegrini et al. 2003a; Omma et al. 2004). Indeed, calculations by Körding, Jester \& Fender (2008; see also Heinz, Merloni \& Schwab 2007) show that the total kinetic energy injected by LLAGN jets is very substantial. Given the prominent hard X-ray component in LLAGN spectra, inverse-Compton scattering of the hard photons might also provide another avenue to heat the ambient medium (Ciotti \& Ostriker 2001). Either form of energy injection - mechanical or radiativecan lead to unsteady, intermittent accretion with a short duty cycle.

- Subluminous disk A thin disk can be tolerated if it can be made extremely subluminous during periods of intermittent activity (Shields \& Wheeler 1978). This situation would arise if accretion disks in AGNs undergo the thermal-viscous ionization instability (Lin \& Shields 1986; Siemiginowska, Czerny \& Kostyunin 1996), in which case they spend most of their time in quiescence, punctuated by brief episodes of intense outbursts. Menou \& Quataert (2001) questioned the applicability of the ionization instability in AGNs, but they suggested that low-luminosity systems (with $\dot{M} \lesssim$ $10^{-3} M_{\odot} \mathrm{yr}^{-1}$ ) may contain disks in which mass accumulates in a stable, nonaccreting "dead zone." Others have managed to stall accretion by condensing the hot flow into an inner cold, inert disk (Nayakshin 2003; Tan \& Blackman 2005; Jolley \& Kuncic 2007), which may form naturally from Compton cooling of the corona (Liu et al. 2007). Finally, Merloni \& Fabian (2002) proposed that LLAGNs do contain a cold thin disk, but because of their low mass accretion rates, they liberate a large fraction of their gravitational energy in a strongly magnetized, unbound corona. Since a cold disk component is present in all these models, they face a serious, and in my opinion insurmountable, challenge because LLAGNs generally do not show 
fluorescent $\mathrm{Fe} \mathrm{K} \alpha$ emission or reflection features in their X-ray spectra. The Merloni \& Fabian model may be spared of this criticism, as the disk may be highly ionized, but it does predict strong and rapid X-ray variability, which is generally not observed in LLAGNs (§ 5.3; Ptak et al. 1998).

\subsection{The Disk-Jet Connection}

As the mass accretion rate drops and the radiative efficiency declines, an increasing fraction of the accretion power gets channeled into a relativistic jet whose energy release is mainly kinetic rather than radiative. The principal evidence for the growing importance of jets in LLAGNs comes from the broad-band SEDs, which invariably are prominent in the radio, with the degree of radio-loudness rising systematically (albeit with significant scatter) with decreasing Eddington ratio ( $\S 5.8$; Figure 10b). Where available, VLBI imaging on milliarcsecond scales reveals unresolved cores with nonthermal brightness temperatures and a flat or slightly inverted spectrum - classical signposts of a relativistic jet (Blandford \& Königl 1979). Detailed modeling of the SEDs of individual sources often shows that the accretion flow itself does not produce enough radio emission to match the data: that extra "something else" is most plausibly attributed to the jet component (Quataert et al. 1999; Ulvestad \& Ho 2001b; Fabbiano et al. 2003; Pellegrini et al. 2003b; Anderson, Ulvestad \& Ho 2004; Ptak et al. 2004; Wu \& Cao 2005; Nemmen et al. 2006; Wu, Yuan \& Cao 2007). Moreover, RIAF models predict radio spectral indices of $\alpha \approx+0.4$ (Mahadevan 1997), whereas the observed values more typically fall in the range $\alpha \approx-0.2$ to +0.2 .

The jet may contribute substantially outside of the radio band, especially in the optical and X-rays. Some advocate that the jet, in fact, accounts for most or even all of the emission across the broad-band SED. For example, Yuan et al. (2002) successfully fitted the multiwavelength data of NGC 4258 with effectively a jet-only model. In their picture, a radiative shock at the base of the jet gives rise to synchrotron emission in the near-IR and optical regions, whose self-Compton component then explains the X-rays; the flat-spectrum radio emission comes from further out in the jet. Similar models have been devised for the Galactic Center source Sgr A* (Falcke \& Markoff 2000; Yuan, Markoff \& Falcke 2002). The gross similarity between the SEDs of some FR I nuclei and BL Lac objects, which are jet-dominated sources but otherwise also low-accretion rate systems (Wang, Staubert \& Ho 2002), has also been noted (e.g., Bower et al. 2000; Capetti et al. 2000; Chiaberge et al. 2003; Meisenheimer et al. 2007).

Statistical samples that are larger but more limited in spectral coverage have come from combining radio data with high-resolution optical or X-ray observations. Studies that specifically target radio galaxies, particularly FR I sources and weak-line FR IIs, report that the core radio power scales tightly with the optical and/or X-ray continuum luminosity, a finding often taken to support a common nonthermal, jet origin for the broad-band emission (Worrall \& Birkinshaw 1994; Canosa et al. 1999; Chiaberge, Capetti \& Celotti 1999, 2000; Capetti et al. 2002; Verdoes Kleijn et al. 2002; Donato, Sambruna \& Gliozzi 2004; Balmaverde \& Capetti 2006; Balmaverde, Capetti \& Grandi 2006; Evans et al. 2006; for a counterargument, see Rinn, Sambruna \& Gliozzi 2005 and Gliozzi et al. 2008). A similar radio-optical correlation, after correcting for Doppler boosting, is also seen among BL Lac objects (Giroletti et al. 2006), strengthening the case that FR I radio galaxies and BL Lac objects are intrinsically the same but 
misoriented siblings. Many FR II systems, on the other hand, especially those with broad lines, deviate systematically from the baseline FR I correlations, by exhibiting stronger optical and X-ray emission for a given level of radio emission (Chiaberge, Capetti \& Celotti 2000, 2002; Varano et al. 2004). In concordance with the frequent detection of X-ray absorption and Fe $\mathrm{K} \alpha$ emission (Evans et al. 2006), this suggests that FR IIs have higher accretion rates and a much more dominant accretion flow component, relative to the jet, than FR Is.

Any attempt to explain the broad-band spectrum of LLAGNs with either just a RIAF or just a jet runs the risk of oversimplification. Clearly both are required. The trick is to figure out a reliable way to divvy up the two contributions to the SED. We cannot deny that there is a jet, because we see it directly in the radio at a strength far greater than can be attributed to the RIAF. The jet emission must contribute at some level outside of the radio band. At the same time, the jet cannot exist in isolation; it is anchored to and fed by some kind of accretion flow, of which a promising configuration is a vertically thick RIAF (Livio, Ogilvie \& Pringle 1999; Meier 1999). An outstanding problem is that the interpretation of the data is not unique. Because many of the model parameters are poorly constrained and the broad-band data remain largely fragmentary and incomplete, the SEDs often can be fit with pure jet models, pure accretion flow models, or some combination of the two. The recent detection of high levels of polarization in the optical nuclei of FR Is (Capetti et al. 2007) strongly points toward a synchrotron origin in the jet for the optical continuum, but even this observation cannot be considered definitive, because a RIAF can also produce nonthermal flares (e.g., in Sgr A*; Quataert 2003).

The so-called BH fundamental plane - a nonlinear correlation among radio luminosity, X-ray luminosity, and BH mass - offers a promising framework to unify accreting BHs over a wide range in mass and accretion rates. Merloni, Heinz \& Di Matteo (2003) first demonstrated that the correlation between $L_{\mathrm{rad}}$ and $L_{\mathrm{X}}$ tightens considerably after including $M_{\mathrm{BH}}$ as a third variable. Combining observational material for several Galactic stellar BHs and a large sample of nearby LLAGNs, they find that

$$
\log L_{\mathrm{rad}}=0.60 \log L_{\mathrm{X}}+0.78 \log M_{\mathrm{BH}} .
$$

This empirical correlation agrees well with the theoretical relations between radio flux, BH mass, and accretion rate derived from the scale-invariant disk-jet model of Heinz \& Sunyaev (2003). The BH fundamental plane, however, appears to be a very blunt tool. In an independent analysis, Falcke, Körding \& Markoff (2004) obtained a similar empirical relation, but unlike Merloni, Heinz \& Di Matteo these authors explained the scaling coefficients entirely in terms of a jet-dominated model. Moreover, as emphasized by Körding, Falcke \& Corbel (2006), objects with very different emission processes, including luminous quasars and BL Lac objects, sit on the same correlation, albeit with larger scatter.

I illustrate this point in Figure 12a, which includes all Palomar LLAGNs with suitable data, along with the collection of high-luminosity sources from L.C. Ho (in preparation). With the exception of a handful of radio-loud quasars, the vast majority of the objects fall on a well-defined swath spanning $\sim 10$ orders of magnitude in luminosity. There are no obvious differences among the various subclasses of LLAGNs, except that the type 1 sources appear more tightly correlated. Plotting the residuals of the fundamental plane relation versus the Eddington ratio reveals two interesting points (Figure 12b). First, although the 

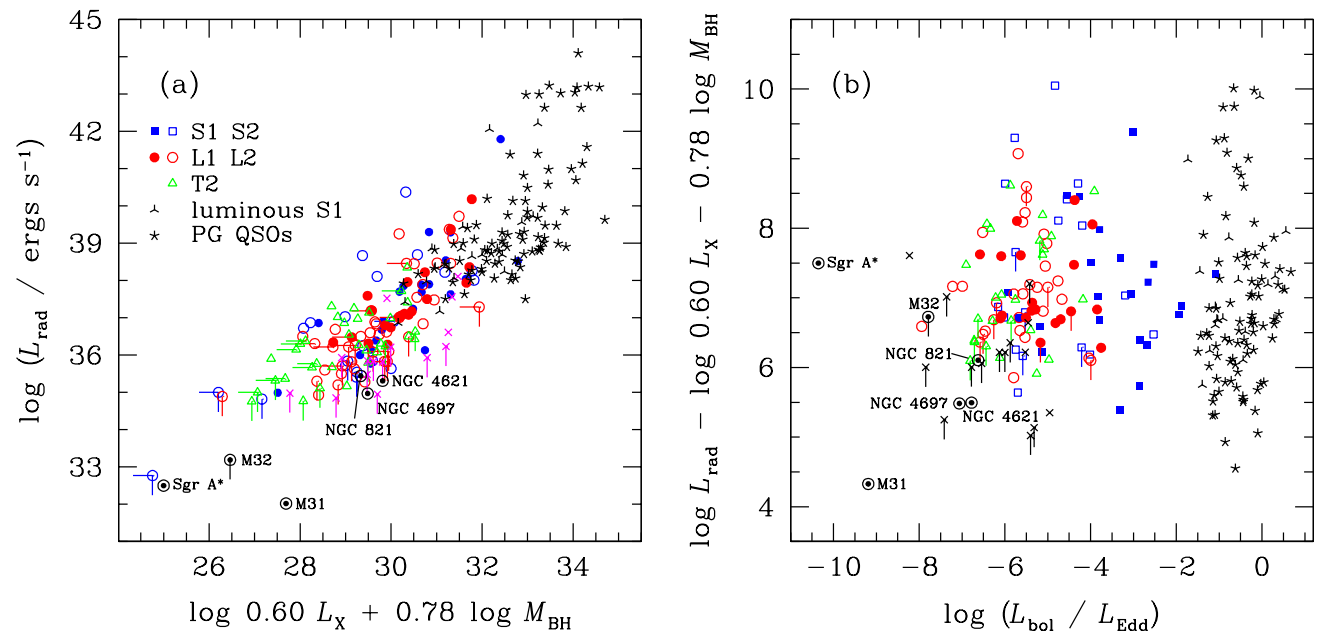

Figure 12: (a) Fundamental plane correlation among core radio luminosity, Xray luminosity, and $\mathrm{BH}$ mass. (b) Deviations from the fundamental plane as a function of Eddington ratio.

intrinsic scatter of the relation is quite large, it markedly increases for objects with high Eddington ratios, at $L_{\mathrm{bol}} / L_{\mathrm{Edd}} \approx 10^{-1 \pm 1}$, as already noted by Maccarone, Gallo \& Fender (2003) and Merloni, Heinz \& Di Matteo (2003). The scatter flares up because the radio-loud quasars lie offset above the relation and the radio-quiet quasars on average lie offset below the relation. At the opposite extreme, sources with $L_{\text {bol }} / L_{\text {Edd }} \lesssim 10^{-6.5}$ may also show a systematic downturn, in possible agreement with the proposal by Yuan \& Cui (2005) that below a critical threshold, $L_{\mathrm{X}} \approx 10^{-5.5} L_{\mathrm{Edd}}$, both the radio and the $\mathrm{X}$-rays should be dominated by emission from the jet. M31 (Garcia et al. 2005), NGC 821 (Pellegrini et al. 2007), and NGC 4621 and NGC 4697 (Wrobel, Terashima \& Ho 2008) seem to conform to Yuan \& Cui's prediction, but M32 and especially Sgr A* clearly do not. Additional deep radio and X-ray observations of ultra-low-luminosity nuclei would be very valuable to clarify the situation in this regime.

If, as surmised, the relative proportions between jet and disk output depend on accretion rate, with the bulk of the radiated power, even in the X-rays, originating from the former in the lowest accretion rate systems, two important consequences ensue. With respect to the microphysics of RIAFs, it implies that the radiative efficiencies are even lower than previously inferred under the assumption that the X-rays emanate solely from the accretion flow. On a more global, environmental scale, shifting the emphasis from the disk to the jet changes the balance between kinetic versus radiative output, with important implications for prescriptions of AGN feedback in models of galaxy formation because BHs spend most of their lives in a low-state. From empirical and theoretical considerations (Heinz, Merloni \& Schwab 2007; Körding, Jester \& Fender $2008)$, the jet carries a substantial fraction of the accreted rest mass energy: $P_{\text {jet }} \approx 0.2 \eta \dot{M} c^{2} \approx 7.2 \times 10^{36}\left(L_{\mathrm{rad}} / 10^{30} \mathrm{ergs} \mathrm{s}^{-1}\right)^{12 / 17} \mathrm{ergs} \mathrm{s}^{-1}$. In fact, the total kinetic energy injected by LLAGN jets is comparable to or perhaps even greater than the contribution from supernovae. At low redshifts, radiative feedback from quasars, which is commonly assumed to operate with an efficiency of $\sim 5 \%$, may 


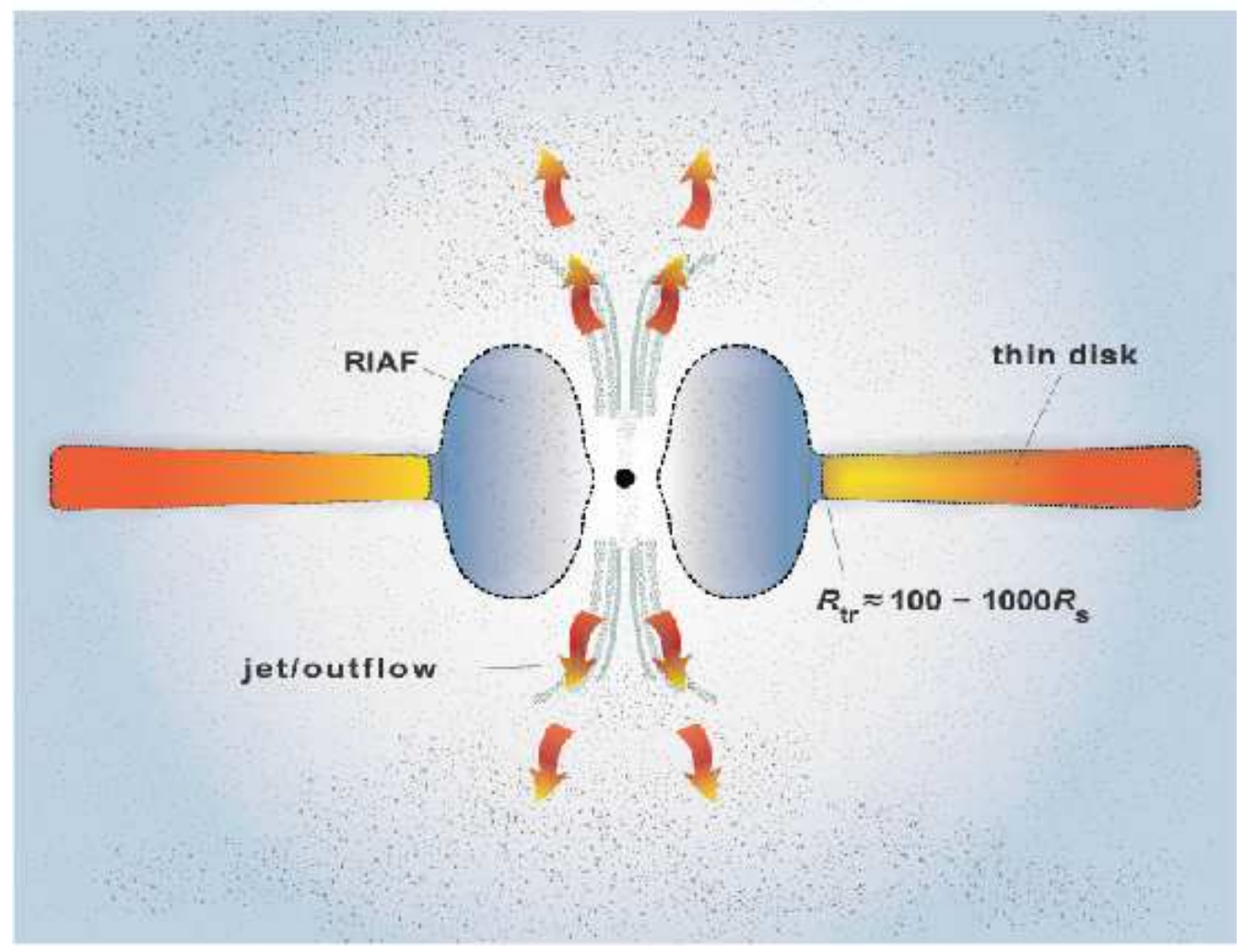

Figure 13: A cartoon of the central engine of LLAGNs, consisting of three components: an inner, radiatively inefficient accretion flow (RIAF), an outer, truncated thin disk, and a jet or outflow. (Courtesy of S. Ho.)

be less important then jet-driven feedback from LLAGNs (Körding, Jester \& Fender 2008).

\subsection{The Central Engine of LLAGNs}

The preceding sections argue that the weak nuclear activity seen in the majority of nearby galaxies traces low-level $\mathrm{BH}$ accretion akin to the more familiar form observed in powerful AGNs. However, multiple lines of evidence indicate that LLAGNs are not simply scaled-down versions of their more luminous cousins. They are qualitatively different. From the somewhat fragmentary clues presented in this review, we can piece together a schematic view of the structure of the central engine in LLAGNs (Ho 2002b, 2003, 2005). As sketched in Figure 13, it has three components.

1. Radiatively inefficient accretion flow In the present-day Universe, and especially in the centers of big bulges, the amount of material available for accretion is small, resulting in mass accretion rates that fall far below $10^{-2} \dot{M}_{\text {Edd }}$. In such a regime, the low-density, tenuous material is optically thin and cannot cool efficiently. Rather than settling into a classical optically thick, geometrically thin, radiatively efficient disk - the normal configuration for luminous AGNs - the accretion flow puffs up into a hot, quasi-spherical, radiatively inefficient distribution, whose dynamics may be 
dominated by advection, convection, or outflows. This is an area of active ongoing theoretical research. In the interest of brevity, I will gloss over the technical details and simply follow Quataert (2003) by calling these RIAFs. The existence of RIAFs, or conversely the absence of a standard thin disk extending all the way to small radii (a few Schwarzschild radii $R_{\mathrm{S}}$ ), is suggested by the feeble luminosities of LLAGNs, by their low Eddington ratios, and especially by their low inferred radiative efficiencies. The great disparity between the available fuel supply and the actually observed accretion luminosity demands that the radiative efficiency of the accretion flow be much less than $\eta=0.1$ ( $\S 8.1$ ). Additional support for RIAFs comes from considerations of the SED, particularly the absence of the big blue bump, a classical signature of the thin disk, and the preponderance of intrinsically hard X-ray spectra.

2. Truncated thin disk Beyond a transition radius $R_{\mathrm{tr}} \approx 100-1000 R_{\mathrm{S}}$, the RIAF switches to a truncated optically thick, geometrically thin disk. The observational evidence for this component comes in three forms. First, the SEDs of some well-studied LLAGNs require a truncated thin disk to explain the big red bump - the prominent mid-IR peak and the steep fall-off of the spectrum in the optical-UV region $(\S 5.8)$. The thermal disk emission is cool (red) not only because of a low accretion rate (Lawrence 2005) but also because the inner radius of the disk does not extend all the way in to a few $R_{\mathrm{S}}$ as in luminous AGNs. Second, the very same truncated disk structure employed to model the SED simultaneously accounts for the relativistically broadened, double-peaked emission-line profiles observed in some sources ( $§ 5.5$ ). Indeed, in the case of NGC 1097 (Nemmen et al. 2006), the transition radius derived from modeling the $\operatorname{SED}\left(R_{\mathrm{tr}}=225 R_{\mathrm{S}}\right)$ agrees remarkably well with the inner radius of the disk obtained from fitting the double-peaked broad $\mathrm{H} \alpha$ profile. Ho et al. (2000) suggested that doublepeaked broad emission lines are commonplace in LLAGNs. By implication, the truncated disk configuration inferred from this special class of line profiles must be commonplace too. Lastly, the striking absence of broad Fe $\mathrm{K} \alpha$ emission in the X-ray spectra of LLAGNs $(\S 5.3)$, a feature commonly attributed to X-ray fluorescence off of a cold accretion disk extending inward to a few $R_{\mathrm{S}}$ in bright Seyfert 1 nuclei (e.g., Nandra et al. 1997b, 2007), strongly suggests that in low-luminosity sources such a structure is either absent or truncated interior to some radius, such that it subtends a significantly smaller solid angle. Similar lines of reasoning have been advanced for broad-line radio galaxies that show weak Fe K $\alpha$ emission and weak Compton reflection (Woźniak et al. 1998; Eracleous, Sambruna \& Mushotzky 2000; Lewis et al. 2005), although these characteristics can be mimicked by an ionized but otherwise untruncated disk (Ballantyne, Ross \& Fabian 2002).

3. Jet/outflow The empirical connection between LLAGNs and jets has been established unequivocally from radio observations. Not only are the SEDs of LLAGNs generically radio-loud, but the strength of the radio emission generally cannot be fit without recourse to a jet component, which in many cases can be seen directly from VLBI-scale radio images. From a theoretical point of view, jets may share a close physical connection with RIAFs. As emphasized by Narayan \& Yi (1995) and Blandford \& Begelman (1999), RIAFs have a strong tendency to drive bipolar outflows due to the high 
thermal energy content of the hot gas. Whether such outflows can develop into highly collimated, relativistic ejections remains to be seen, but they at least provide a promising starting point. RIAFs may be additionally conducive to jet formation because its vertically thick structure enhances the large-scale poloidal component of the magnetic field, which plays a critical role in launching jets (Livio, Ogilvie \& Pringle 1999; Meier 1999; Ballantyne \& Fabian 2005; Ballantyne 2007). It is interesting to recall that the original motivation for ion-supported tori (Rees et al. 1982), an early incarnation of RIAFs, was to explain the low luminosity of radio galaxies. Rees et al. postulated that the puffed-up structure of the ion torus may help facilitate the collimation of the jet.

The above-described three-component structure has been applied to model the broad-band spectrum of a number of LLAGNs, including NGC 4258 (Lasota et al. 1996; Gammie, Narayan \& Blandford 1999), M81 and NGC 4579 (Quataert et al. 1999), NGC 3998 (Ptak et al. 2004), and NGC 1097 (Nemmen et al. 2006). For the handful of LLAGNs with available estimates of the transition radii, $R_{\text {tr }}$ seems to scale roughly inversely with $L_{\text {bol }} / L_{\text {Edd }}$ (Yuan \& Narayan 2004). This trend may be in agreement with models for disk evaporation (Liu \& Meyer-Hofmeister 2001). As the latter authors note, however, disks attain their maximum evaporation efficiency at $R_{\mathrm{tr}} \approx 300 R_{\mathrm{S}}$, making sources such as M81 and NGC 4579, both with $R_{\mathrm{tr}} \approx 100 R_{\mathrm{S}}$ (Quataert et al. 1999), difficult to explain. At a qualitative level, at least, the general idea that the thin disk recedes to larger and larger radii as the accretion rate drops is probably correct. In an analysis of $33 \mathrm{PG}$ quasars with Fe K $\alpha$ emission detected in XMM-Newton spectra, Inoue, Terashima \& Ho (2007) find that the iron line profile varies systematically with Eddington ratio. Specifically, the $\mathrm{Fe} \mathrm{K} \alpha$ profile becomes narrower with decreasing $L_{\text {bol }} / L_{\text {Edd }}$, a result that can be interpreted as a systematic increase in the inner radius of the accretion disk at low accretion rates.

The basic schematic proposed in Figure 13 is hardly new. To my knowledge, a hybrid model consisting of a RIAF - then called an ion-supported torus - plus a truncated thin disk was most clearly articulated in a prescient paper by Chen \& Halpern (1989) in their description of Arp 102B, later elaborated by Eracleous \& Halpern (1994) in the general context of double-peaked broad-line radio galaxies. Chen \& Halpern identified the $25 \mu \mathrm{m}$ peak in the SED with the turnover frequency of the synchrotron peak from the RIAF, whose elevated structure illuminates an outer thin disk that emits the double-peaked broad optical lines. The overall weakness of the UV continuum in Arp 102B (Halpern et al. 1996) further corroborates a truncated thin disk structure and potentially provides an explanation for the low-ionization state of the emission-line spectrum. As for the jet component, it was assumed to be present, at least implicitly, insofar as the double-peaked broad-line AGNs were thought to reside preferentially in radio-loud AGNs, and the very concept of ion-supported tori was invented with reference to radio galaxies (Rees et al. 1982).

Recent developments add important refinements and modifications to Chen \& Halpern's original picture. First, the mid-IR peak in most objects is dominated by thermal emission from the truncated thin disk rather than by the synchrotron peak of the RIAF. Second, the jet component, which was once regarded as somewhat incidental, has emerged as a natural and perhaps inevitable outgrowth of the inner accretion flow itself. Third, although the original model was invented 
to explain a small minority of the AGN population (double-peaked radio-loud sources), now we have good reason to believe that similar physical conditions prevail in LINERs as a class (Ho et al. 2000), and, by extension, in the majority of nearby accreting BHs.

The physical picture outlined above for LLAGNs shares strong similarities with that developed for X-ray binaries in their hard state (see Maccarone, Fender \& Ho 2005 and references therein), suggesting that the basic architecture of the central engine around accreting BHs - across 10 orders of magnitude in mass - is essentially scale-invariant (Meier 2001; Maccarone, Gallo \& Fender 2003; Merloni, Heinz \& Di Matteo 2003; Falcke, Körding \& Markoff 2004; Ho 2005; Körding, Jester \& Fender 2006).

\section{CONCLUDING REMARKS}

The topics covered in this article are both very old and very new. It has been known for over three decades that a large segment of the galaxy population exhibits signs of unusual activity in their nuclei, and for nearly as long people have puzzled over the physical origin of this activity. Over time the observational material at optical wavelengths has improved markedly, especially with the completion of the Palomar survey, but the debate has only intensified. Given their abundance, most of the controversy has centered, not surprisingly, on LINERs. While the nonstellar nature of a sizable fraction of LINERs is now incontrovertible (e.g., those with broad $\mathrm{H} \alpha$ emission), the AGN content in the majority of the class remains unsettled. Determining the physical origin of these systems is more than of mere phenomenological interest. Because LINERs are so numerousbeing the dominant constituent of the local LLAGN population and a sizable fraction of all galaxies - they have repercussions on virtually every issue related to AGN and BH demographics.

The main source of contention stems from the fact that Mother Nature knows too many ways of generating nebular conditions that qualitatively look similar to the low-ionization characteristics of LINERs at optical wavelengths. A dizzying array of excitation mechanisms has been proposed to explain LINERs, ranging from variants of conventional AGN photoionization, to shocks of various flavors, to interstellar processes such as cooling flows and turbulent mixing layers, to stellar-based photoionization by populations both young and old, prosaic and exotic. This field has suffered not from a shortage of ideas, but from too many. As a consequence, whenever LINERs are discussed, it is customary to end with the pessimistic mantra that they are a mixed-bag, heterogeneous class of objects, a statement with somewhat dismissive connotations that is often taken to mean that we have no idea what they are and that they are too messy to deal with. This is an unfair characterization of the progress that has been made, and I think that there is good reason to sound a more positive note.

As summarized in this review, a number of developments during the last few years shed considerable light on the physical origin of LLAGNs in general and LINERs in particular. The key advances have come from the broader perspective afforded by observations outside of the traditional optical window, especially in the radio and X-rays, although important insights can also be credited to optical and UV data taken with HST. In all instances, high angular resolution has been a critical factor to disentangle the weak nuclear emission from the blinding host 
galaxy background.

Other developments have been instrumental in forging a coherent view of nuclear activity in the nearby Universe. On the theoretical side, rapid advances in the study of radiatively inefficient accretion flows, originally primarily motivated by applications to X-ray binaries and to the Galactic Center source Sgr A*, has led to a growing appreciation that they are also relevant to LLAGNs in general. Many investigators have sharpened the physical analogy between the spectral states of X-ray binaries and certain classes of AGNs, an effort that has resulted in a more holistic picture of $\mathrm{BH}$ accretion, especially as it concerns the evolution of the accretion flow in response to variations in mass accretion rate and the mechanism for generating jets or outflows. Meanwhile, the dynamical detection of supermassive BHs, their ubiquity, and the discovery of scaling relations between BHs and their host galaxies have given a major boom to studies of AGNs in all their multi-faceted manifestations. More than ever, in the grand scheme of things, AGNs are no longer viewed as rare and exotic oddities but as natural episodes during the life cycle of galaxies during which their BHs accrete, grow, and shine. The impact of BH growth and AGN feedback have emerged forcefully as major new themes in galaxy formation. LLAGNs gain an even greater prominence within this context. Although the bulk of the mass density of BHs was accreted in a luminous, radiatively efficient mode, it behooves us to understand how BHs spend most of their lives. The detection of supermassive BHs has also fundamentally altered the character of the discourse on LLAGNs. We can now shift our attention from the question of whether LLAGNs contain BHs - an implicit or explicit motivation for much of the past discussion on the nature of these sources - to why these BHs have the properties that they do. Among other things, LLAGNs can be used as an effective platform for exploring accretion physics in highly sub-Eddington systems and for investigating physical processes in the circumnuclear regions of galaxies that are normally masked by brighter nuclei.

The following is a list of the "top ten" results from this paper.

1. Approximately $2 / 3$ of local E-Sb galaxies exhibit weak nuclear activity incompatible with normal stellar processes; in contrast, only about $15 \%$ of Sc-Sm galaxies show AGN activity $(\S 3)$.

2. The vast majority of LINERs, and, by implication, most nearby weakly active nuclei, are genuine, accretion-powered AGNs ( $\S 6.1,6.5)$.

3. The ubiquity of LLAGNs in galaxies with bulges strongly supports the current paradigm derived from dynamical studies that all bulges contain BHs. However, the detection of AGNs in some bulgeless, even dwarf, galaxies proves that bulges are not necessary for the formation of central BHs ( $\$ 7)$.

4. The luminosity function of nearby LLAGNs follows $\Phi \propto L^{-1.2 \pm 0.2}$ from $L_{\mathrm{H} \alpha} \approx 3 \times 10^{41}$ to $10^{38} \mathrm{ergs} \mathrm{s}^{-1}$, below which it appears to flatten down to $L_{\mathrm{H} \alpha} \approx 6 \times 10^{36} \mathrm{ergs} \mathrm{s}^{-1}$ or $M_{B} \approx-8 \mathrm{mag}(\S 5.9)$.

5. Stellar photoionization by young or intermediate-stars and shock heating can be ruled out as the excitation mechanisms for LLAGNs $(\S \S 6.2,6.3)$.

6. Despite the overall success of AGN photoionization models, many LLAGNs, especially type 2 sources, have a shortage of ionizing photons. The energy deficit problem could be solved with cosmic ray heating and extra ionization 
from evolved (post-AGB) stars, diffuse thermal plasma, and the cumulative $\mathrm{X}$-ray emission from low-mass X-ray binaries (§6.4).

7. Variations in the mass accretion rate give rise to the different classes of emission-line nuclei. LINERs are the low-luminosity, low-accretion rate extension of Seyferts, followed by transition nuclei, and ending with absorptionline nuclei at the end of the $\mathrm{BH}$ starvation sequence $(\S 6.5)$.

8. LLAGNs are not simply scaled-down versions of powerful AGNs. Their central engines undergo fundamental changes when the accretion rate drops to extremely sub-Eddington values. In this regime, the BLR and obscuring torus disappear $(\S \S 5.5,5.6)$. LLAGNs do not follow the standard AGN unification model.

9. Below a characteristic luminosity of $\sim 1 \%$ Eddington, the canonical optically thick, geometrically thin accretion disk transforms into a three-component structure consisting of an inner vertically thick and radiatively inefficient accretion flow, a truncated outer thin disk, and a jet or outflow $(\S \S 5.8,8.3)$.

10. At the lowest accretion rates, an increasing fraction of the accretion energy gets channeled into a relativistic jet. The emitted energy is mainly kinetic rather than radiative. Since radiation and kinetic jets interact differently with the surrounding gas, this has important implications for AGN feedback into galaxy formation $(\S 8.2)$.

My research is supported by the Carnegie Institution of Washington and by NASA grants from the Space Telescope Science Institute (operated by AURA, Inc., under NASA contract NAS5-26555). I would like to recognize my collaborators who have contributed to the work covered in this review, especially A.J. Barth, M. Eracleous, M.E. Filho, A.V. Filippenko, J.E. Greene, D. Maoz, E.C. Moran, C.Y. Peng, A. Ptak, E. Quataert, H.-W. Rix, W.L.W. Sargent, M. Sarzi, J.C. Shields, Y. Terashima, J.S. Ulvestad, and J.M. Wrobel. Several of them (A.J. Barth, M. Eracleous, J.E. Greene, M. Sarzi, J.C. Shields, Y. Terashima) read an early draft of the manuscript and provided useful feedback that helped to improve it. Some of the concepts expressed in the review were sharpened after correspondence with M. Eracleous, G. Ferland, J.C. Shields, and Y. Terashima. I thank A.J. Barth, A.V. Filippenko, and W.L.W. Sargent for permission to cite material in advance of publication, H.M.L.G. Flohic and M. Eracleous for providing the images for Figure 5, and Salvador Ho for drafting Figure 13. I am grateful to J. Kormendy for his steadfast encouragement, wise counsel, and meticulous editing.

\section{LITERATURE CITED}

Aldrovandi SMV, Péquignot D. 1973. Astron. Astrophys. 26:33

Allen MG, Dopita, MA, Tsvetanov ZI. 1998. Ap. J. 493:571

Alonso-Herrero A, Rieke MJ, Rieke GH, Ruiz M. 1997. Ap. J. 482:74

Alonso-Herrero A, Rieke MJ, Rieke GH, Shields JC. 2000. Ap. J. 530:688

Anderson JM, Ulvestad JS. 2005. Ap. J. 627:674

Anderson JM, Ulvestad JS, Ho LC. 2004. Ap. J. 603:42

Antonucci R. 1993. Annu. Rev. Astron. Astrophys. 31:473

Armus L, Heckman TM, Miley GK. 1990. Ap. J. 364:471 
Atkinson JW, Collett JL, Marconi A, Axon DJ, Alonso-Herrero A, et al. 2005. MNRAS 359:504

Avni Y, Tananbaum H. 1982. Ap. J. 262:L17

Awaki H, Mushotzky R, Tsuru T, Fabian AC, Fukazawa Y, et al. 1994. PASJ 46:L65

Baganoff FK, Maeda Y, Morris M, Bautz MW, Brandt WN, et al. 2003. Ap. J. 591:891

Baldwin JA, Phillips MM, Terlevich R. 1981. PASP 93:5

Ballantyne DR. 2007. Mod. Phys. Lett. A 22:2397

Ballantyne DR, Fabian AC. 2005. Ap. J. 622:L97

Ballantyne DR, Ross RR, Fabian AC. 2002. MNRAS 332:L45

Balmaverde B, Capetti A. 2006. Astron. Astrophys. 447:97

Balmaverde B, Capetti A, Grandi P. 2006. Astron. Astrophys. 451:35

Barger AJ, Cowie LL, Mushotzky RF, Richards EA. 2001. Astron. J. 121:662

Barth AJ. 2004. In Carnegie Observatories Astrophysics Series, Vol 1: Coevolution of Black Holes and Galaxies, ed. LC Ho, p. 21. Cambridge: Cambridge Univ. Press

Barth AJ, Filippenko AV, Moran EC. 1999a. Ap. J. 515:L61

Barth AJ, Filippenko AV, Moran EC. 1999b. Ap. J. 525:673

Barth AJ, Greene JE, Ho LC. 2005. Ap. J. 619:L151

Barth AJ, Ho LC, Filippenko AV. 2003. In Active Galactic Nuclei: from Central Engine to Host Galaxy, ed. S Collin, F Combes, I Shlosman, p. 387. San Francisco: ASP

Barth AJ, Ho LC, Filippenko AV, Rix H-W, Sargent WLW 2001a. Ap. J. 546:205

Barth AJ, Ho LC, Filippenko AV, Sargent WLW. 1998. Ap. J. 496:133

Barth AJ, Ho LC, Rutledge RE, Sargent WLW. 2004. Ap. J. 607:90

Barth AJ, Reichert GA, Filippenko AV, Ho LC, Shields JC, Mushotzky RF, Puchnarewicz EM. 1996. Astron. J. 112:1829

Barth AJ, Reichert GA, Ho LC, Shields JC, Filippenko AV, Puchnarewicz EM. 1997. Astron. J. 114:2313

Barth AJ, Sarzi M, Rix H-W, Ho LC, Filippenko AV, Sargent WLW. 2001b. Ap. J. 555:685

Barth AJ, Shields JC. 2000. PASP 112:753

Barth AJ, Tran H, Brotherton MS, Filippenko AV, Ho LC, et al. 1999. Astron. J. 118:1609

Bassani L, Dadina M, Maiolino R, Salvati M, Risaliti G, et al. 1999. Ap. J. Suppl. 121:473

Begelman MC, Fabian AC. 1990. MNRAS 244:36P

Bendo GJ, Dale DA, Draine BT, Engelbracht CW, Kennicutt RC Jr, et al. 2006. Ap. J. 645:134

Bendo GJ, Joseph RD. 2004. Astron. J. 127:3338

Bennert N, Jungwiert B, Komossa S, Haas M, Chini R. 2006. Astron. Astrophys. 456:953

Bianchi S, Corral A, Panessa F, Barcons X, Matt G, et al. 2008. MNRAS 385:195

Bietenholz MF, Bartel N, Rupen NP. 2000. Ap. J. 532:895

Binette L. 1985. Astron. Astrophys. 143:334

Binette L, Magris CG, Stasińska G, Bruzual AG. 1994. Astron. Astrophys. 292:13

Binney J, Tabor G. 1995. MNRAS 276:663

Blandford RD, Begelman MC. 1999. MNRAS 303:L1

Blandford RD, Königl A. 1979. Ap. J. 232:34 
Blanton EL, Sarazin CL, Irwin JA. 2001. Ap. J. 552:106

Böhringer H, Belsole E, Kennea J, Matsushita K, Molendi S, et al. 2001. Astron. Astrophys. 365::181

Boisson C, Joly M, Moultaka J, Pelat D, Serote-Roos M. 2000. Astron. Astrophys. $357: 850$

Böker T, van der Marel RP, Laine S, Rix H-W, Sarzi M, Ho LC, Shields JC. 2002. Astron. J. 123:1389

Bonatto C, Bica E, Alloin D. 1989. Astron. Astrophys. 226:23

Bondi H. 1952. MNRAS 112:195

Bower GA, Green RF, Quillen AC, Danks A, Gull T, et al. 2000. Ap. J. 534:189

Bower GA, Wilson AS, Heckman TM, Richstone DO. 1996. Astron. J. 111:1901

Burbidge EM, Burbidge G. 1962. Ap. J. 135:694

Burbidge EM, Burbidge G. 1965. Ap. J. 142:634

Burbidge G, Gould RJ, Pottasch SR. 1963. Ap. J. 138:945

Canosa CM, Worrall DM, Hardcastle MJ, Birkinshaw M. 1999. MNRAS 310:30

Capetti A, Axon DJ, Chiaberge M, Sparks WB, Macchetto FD, et al. 2007. Astron. Astrophys. 471:137

Capetti A, Celotti A, Chiaberge M, de Ruiter HR, Fanti R, et al. 2002. Astron. Astrophys. 383:104

Capetti A, Trussoni E, Celotti A, Feretti L, Chiaberge M. 2000. MNRAS 318:493

Capetti A, Verdoes Kleijn G, Chiaberge M. 2005. Astron. Astrophys. 439:935

Cappellari M, Bertola F, Burstein D, Buson LM, Greggio L, Renzini A. 2001. Ap. J. 551:197

Cappi M, Panessa F, Bassani L, Dadina M, Dicocco G, et al. 2006. Astron. Astrophys. 446:459

Carollo CM, Stiavelli M, de Zeeuw PT, Mack, J. 1997. Astron. J. 114:2366

Carrillo R, Masegosa J, Dultzin-Hacyan D, Ordoñez R. 1999. Rev. Mex. Astron. Astrof. 35:187

Carswell RF, Baldwin JA, Atwood B, Phillips MM. 1984. Ap. J. 286:464

Chary R, Becklin EE, Evans AS, Neugebauer G, Scoville NZ, et al. 2000. Ap. J. 531:756

Chen K, Halpern JP. 1989. Ap. J. 344:115

Chiaberge M, Capetti A, Celotti A. 1999. Astron. Astrophys. 349:77

Chiaberge M, Capetti A, Celotti A. 2000. Astron. Astrophys. 355:873

Chiaberge M, Capetti A, Celotti A. 2002. Astron. Astrophys. 394:791

Chiaberge M, Capetti A, Macchetto FD. 2005. Ap. J. 625:716

Chiaberge M, Gilli R, Capetti A, Macchetto FD. 2003. Ap. J. 597:166

Chiaberge M, Macchetto FD, Sparks W, Capetti A, Allen MG, Martel AR. 2002. Ap. J. 571:247

Cid Fernandes R Jr, Golzález Delgado RM, Schmitt H, Storchi-Bergmann T, Martins LP, et al. 2004. Ap. J. 605:105

Ciotti L, D'Ercole A, Pellegrini S, Renzini A. 1991. Ap. J. 376:380

Ciotti L, Ostriker JP. 2001. Ap. J. 551:131

Colbert EJM, Mushotzky RF. 1999. Ap. J. 519:89

Colina L, Golzález Delgado RM, Mas-Hesse JM, Leitherer C. 2002. Ap. J. 579:545

Collins JA, Rand RJ. 2001. Ap. J. 551:57

Combes F. 2003. In Active Galactic Nuclei: from Central Engine to Host Galaxy, ed. S Collin, F Combes, I Shlosman, p. 411. San Francisco: ASP

Constantin A, Vogeley MS. 2006. Ap. J. 650:727

Contini M. 1997. Astron. Astrophys. 323:71 
Corbett EA, Kewley L, Appleton PN, Charmandaris V, Dopita MA, et al. 2003. Ap. J. 583:670

Costero R, Osterbrock DE. 1977. Ap. J. 211:675

Dale DA, Smith JDT, Armus L, Buckalew BA, Helou G, et al. 2006. Ap. J. 646:161

Danziger IJ, Fosbury RAE, Penston MV. 1977. MNRAS 179:41P

Decarli R, Gavazzi G, Arosio I, Cortese L, Boselli A, et al. 2007. MNRAS 381:136

Demoulin-Ulrich M-H, Butcher HR, Boksenberg A. 1984. Ap. J. 285:527

Desroches L-B, Ho LC. 2008. Ap. J., submitted

Dewangan GC, Griffiths RE, Di Matteo T, Schurch NJ. 2004. Ap. J. 607:788

Díaz AI, Pagel BEJ, Wilson IRG. 1985. MNRAS 212:737

Díaz AI, Pagel BEJ, Terlevich E. 1985. MNRAS 214:41P

Di Matteo T, Allen SW, Fabian AC, Wilson AS, Young AJ. 2003. Ap. J. 582:133

Di Matteo T, Fabian AC. 1997. MNRAS 286:L50

Di Matteo T, Johnstone RM, Allen SW, Fabian AC. 2001. Ap. J. 550:L19

Disney MJ, Cromwell RH. 1971. Ap. J. 164:L35

Doi A, Kameno S, Kohno K, Nakanishi K, Inoue M. 2005. MNRAS 363:692

Donato D, Sambruna RM, Gliozzi M. 2004. Ap. J. 617:915

Done C., Gierliński M, Sobolewska M, Schurch N. 2007. In The Central Engine of Active Galactic Nuclei, ed. LC Ho, J-M Wang, p. 121. San Francisco: ASP

Dong X-B, Wang T, Yuan W, Shan H, Zhou H, et al. 2007. Ap. J. 657:700

Dopita MA, Koratkar AP, Allen MG, Tsvetanov ZI, Ford HC, et al. 1997. Ap. J. 490:202

Dopita MA, Sutherland RS. 1995. Ap. J. 455:468

Dudik RP, Satyapal S, Gliozzi M, Sambruna RM. 2005. Ap. J. 620:113

Elitzur M, Shlosman I. 2006. Ap. J. 648:L101

Elvis M, Wilkes BJ, McDowell JC, Green RF, Bechtold J, et al. 1994. Ap. J. Suppl. 95:1

Engelbracht CW, Rieke MJ, Rieke GH, Kelley DM, Achtermann JM. 1998. Ap. J. 505:639

Eracleous M, Halpern JP. 1994. Ap. J. Suppl. 90:1

Eracleous M, Halpern JP. 2001. Ap. J. 554:240

Eracleous M, Hwang JA, Flohic HMLG. 2008a. Ap. J. submitted

Eracleous M, Hwang JA, Flohic HMLG. 2008b. Ap. J. submitted

Eracleous M, Sambruna R, Mushotzky RF. 2000. Ap. J. 537:654

Eracleous M, Shields JC, Chartas G, Moran EC. 2002. Ap. J. 565:108

Evans DA, Worrall DM, Hardcastle MJ, Kraft RP, Birkinshaw M. 2006. Ap. J. 642:96

Fabbiano G. 2006. Annu. Rev. Astron. Astrophys. 44:323

Fabbiano G, Baldi A, Pellegrini S, Siemiginowska A, Elvis M, et al. 2004. Ap. J. 616:730

Fabbiano G, Elvis M, Markoff S, Siemiginowska A, Pellegrini S, et al. 2003. Ap. J. 588:175

Fabbiano G, Trinchieri G. 1985. Ap. J. 296:430

Faber SM, Tremaine S, Ajhar EA, Byun Y-I, Dressler A, et al. 1997. Astron. J. 114:1365

Fabian AC, Arnaud KA, Nulsen PEJ, Mushotzky RF. 1986. Ap. J. 305:9

Fabian AC, Canizares CR. 1988. Nature 333:829

Fabian AC, Rees MJ. 1995. MNRAS 277:L55

Falcke H, Körding E, Markoff S. 2004. Astron. Astrophys. 414:895 
Falcke H, Markoff S. 2000. Astron. Astrophys. 362:113

Falcke H, Nagar NM, Wilson AS, Ulvestad JS. 2000. Ap. J. 542:197

Fanaroff BL, Riley JM. 1974. MNRAS 167:31P

Ferrarese L, Merritt D. 2000. Ap. J. 539:L9

Ferland GJ, Mushotzky RF. 1984. Ap. J. 286:42

Ferland GJ, Netzer H. 1983. Ap. J. 264:105

Filho ME, Barthel PD, Ho LC. 2000. Ap. J. Suppl. 129:93

Filho ME, Barthel PD, Ho LC. 2002a. Ap. J. Suppl. 142:223

Filho ME, Barthel PD, Ho LC. 2002b. Astron. Astrophys. 385:425

Filho ME, Barthel PD, Ho LC. 2006. Astron. Astrophys. 451:71

Filho ME, Fraternali F, Nagar NM, Barthel PD, Markoff S, et al. 2004. Astron. Astrophys. 418:429

Filippenko AV. 1985. Ap. J. 289:475

Filippenko AV. 1996. In The Physics of LINERs in View of Recent Observations, ed. M Eracleous, A Koratkar, C Leitherer, LC Ho, p. 17. San Francisco: ASP

Filippenko AV, Halpern JP. 1984. Ap. J. 285:458

Filippenko AV, Ho LC. 2003. Ap. J. 588:L13

Filippenko AV, Ho LC, Sargent WLW. 1993. Ap. J. 410:L75

Filippenko AV, Sargent WLW. 1985. Ap. J. Suppl. 57:503

Filippenko AV, Sargent WLW. 1988. Ap. J. 324:134

Filippenko AV, Sargent WLW. 1989. Ap. J. 342:L11

Filippenko AV, Terlevich R. 1992. Ap. J. 397:L79

Fiore F, ed. 2006. AGNs and Galaxy Evolution. MmSAI 77

Fiore F, Pellegrini S, Matt G, Antonelli LA, Comastri A, et al. 2001. Ap. J. $556: 150$

Flohic HMLG, Eracleous M, Chartas G, Shields JC, Moran EC. 2006. Ap. J. 647:140

Ford HC, Butcher H. 1979. Ap. J. Suppl. 41:147

Fosbury RAE, Melbold U, Goss WM, Dopita MA. 1978. MNRAS 183:549

Fosbury RAE, Melbold U, Goss WM, van Woerden H. 1977. MNRAS 179:89

Fukazawa Y, Iyomoto N, Kubota A, Matsumoto Y, Makishima K. 2001. Astron. Astrophys. 374:73

Gabel JR, Bruhweiler FC. 2002. Astron. J. 124:737

Gabel JR, Bruhweiler FC, Crenshaw DM, Kraemer SB, Miskey CL. 2000. Ap. J. 532:883

Gallimore JF, Axon DJ, O'Dea CP, Baum SA, Pedlar A. 2006. Astron. J. 132:546

Gallo E, Treu T, Jacob J, Woo J-H, Marshall RJ, Antonucci R. 2008. Ap. J. in press

Gammie CF, Narayan R, Blandford RD. 1999. Ap. J. 516:177

Garcia MR, Williams BF, Yuan F, Kong AKH, Primini FA, et al. 2005. Ap. J. 632:1042

Gebhardt K, Bender R, Bower G, Dressler A, Faber SM, et al. 2000. Ap. J. 539:L13

Gebhardt K, Rich RM, Ho LC. 2002. Ap. J. 578:L41

Gebhardt K, Rich RM, Ho LC. 2005. Ap. J. 634:1093

Geha M, Guhathakurta P, van der Marel RP. 2002. Astron. J. 124:3073

Georgantopoulos I, Panessa F, Akylas A, Zezas A, Cappi M, Comastri A. 2002. Astron. Astrophys. 386:60

George IM, Fabian AC. 1991. MNRAS 249:352

Ghosh H, Pogge RW, Mathur S, Martini P, Shields JC. 2007. Ap. J. 656:105 
Giroletti M, Giovannini G, Taylor GB, Falomo R. 2006. Ap. J. 646:801

Gliozzi M, Foschini L, Sambruna RM, Tavecchio F. 2008. Astron. Astrophys. 478:723

Gliozzi M, Sambruna RM, Brandt WN, Mushotzky RF, Eracleous M. 2004. Astron. Astrophys. 413:139

Gliozzi M, Sambruna RM, Foschini L. 2007. Ap. J. 662:878

González Delgado RM, Cid Fernandes R, Peréz E, Martins LP, Storchi-Bergmann T, et al. 2004. Ap. J. 605:127

González-Martín O, Masegosa J, Márquez I, Guerrero MA, Dultzin-Hacyan D. 2006. Astron. Astrophys. 460:45

Granato GL, De Zotti G, Silva L, Bressan A, Danese L. 2004. Ap. J. 600:580

Grandi SA, Osterbrock DE. 1978. Ap. J. 220:783

Greene JE, Ho LC. 2004. Ap. J. 610:722

Greene JE, Ho LC. 2005a. Ap. J. 627:721

Greene JE, Ho LC. 2005b. Ap. J. 630:122

Greene JE, Ho LC. 2006. Ap. J. 641:L21

Greene JE, Ho LC. 2007a. Ap. J. 667:131

Greene JE, Ho LC. 2007b. Ap. J. 670:92

Greene JE, Ho LC, Barth AJ. 2008. Ap. J. submitted

Greene JE, Ho LC, Ulvestad JS. 2006. Ap. J. 636:56

Gronwall C, Jangren A, Salzer JJ, Werk JK, Ciardullo R. 2004. Astron. J. 128:644

Grossan B, Gorjian V, Werner M, Ressler M. 2001. Ap. J. 563:687

Groves BA, Dopita MA, Sutherland RS. 2004. Ap. J. Suppl. 153:75

Groves B, Heckman T, Kauffmann G. 2006. MNRAS 371:1559

Gruenwald RB, Viegas-Aldrovandi SM. 1987. Astron. Astrophys. Suppl. 70:143

Gu Q-S, Huang J-S, Wilson G, Fazio GG. 2007. Ap. J. 671:L105

Guainazzi M, Oosterbroek T, Antonelli LA, Matt G. 2000. Astron. Astrophys. 364:L80

Haas M, Müller SAH, Bertoldi F, Chini R, Egner S, et al. 2004. Astron. Astrophys. 424:531

Halderson EL, Moran EC, Filippenko AV, Ho LC. 2001. Astron. J. 122:637

Hall PB, Yee HKC, Lin H, Morris SL, Patton DR, et al. 2000. Astron. J. 120:2220

Halpern JP, Eracleous M. 1994. Ap. J. 433:L17

Halpern JP, Eracleous M, Filippenko AV, Chen K. 1996. Ap. J. 464:704

Halpern JP, Filippenko AV. 1984. Ap. J. 285:475

Halpern JP, Steiner JE. 1983. Ap. J. 269:L37

Hao L, Strauss MA, Fan XH, Tremonti CA, Schlegel DJ, et al. 2005a. Astron. J. 129:1783

Hao L, Strauss MA, Tremonti CA, Schlegel DJ, Heckman TM, et al. 2005b. Astron. J. 129:1795

Hawkins MRS. 2004. Astron. Astrophys. 424:519

Heckman TM. 1980a. Astron. Astrophys. 87:142

Heckman TM. 1980b. Astron. Astrophys. 87:152

Heckman TM, Balick B, Crane PC. 1980. Astron. Astrophys. Suppl. 40:295

Heckman TM, Baum SA, van Breugel WJM, McCarthy P. 1989. Ap. J. 338:48

Heckman TM, Ptak A, Hornschemeier A, Kauffmann G. 2005. Ap. J. 634:161

Heinz S, Merloni A, Schwab J. 2007. Ap. J. 658:L9

Heinz S, Sunyaev R. 2003. MNRAS 343:L59

Heller CH, Shlosman I. 1994. Ap. J. 424:84

Hernquist L. 1989. Nature. 340;687 
Ho LC. 1996. In The Physics of LINERs in View of Recent Observations, ed. M

Eracleous, A Koratkar, C Leitherer, LC Ho, p. 103. San Francisco: ASP

Ho LC. 1999a. Ap. J. 510:631

Ho LC. 1999b. Ap. J. 516:672

Ho LC. 2002a. Ap. J. 564:120

Ho LC. 2002b. In Issues in Unification of AGNs, ed. R Maiolino, A Marconi, N Nagar, p. 165. San Francisco: ASP

Ho LC. 2003. In Active Galactic Nuclei: from Central Engine to Host Galaxy, ed.

S Collin, F Combes, I Shlosman, p. 379. San Francisco: ASP

Ho LC, ed. 2004a. Carnegie Observatories Astrophysics Series, Vol. 1: Coevolution of Black Holes and Galaxies Cambridge: Cambridge Univ. Press

Ho LC. 2004b. In Carnegie Observatories Astrophysics Series, Vol. 1: Coevolution of Black Holes and Galaxies, ed. LC Ho, p. 293. Cambridge: Cambridge Univ. Press

Ho LC. 2005. In From X-ray Binaries to Quasars: Black Hole Accretion on All Mass Scales, ed. TJ Maccarone, RP Fender, LC Ho, p. 219. Dordrecht: Kluwer

Ho LC, Feigelson ED, Townsley LK, Sambruna RM, Garmire GP, et al. 2001. Ap. J. 549:L51

Ho LC, Filippenko AV. 1993. Astrophys. Space Sci. 205:19

Ho LC, Filippenko AV, Sargent WLW. 1993. Ap. J. 417:63

Ho LC, Filippenko AV, Sargent WLW. 1995. Ap. J. Suppl. 98:477

Ho LC, Filippenko AV, Sargent WLW. 1996. Ap. J. 462:183

Ho LC, Filippenko AV, Sargent WLW. 1997a. Ap. J. Suppl. 112:315

Ho LC, Filippenko AV, Sargent WLW. 1997b. Ap. J. 487:568

Ho LC, Filippenko AV, Sargent WLW. 1997c. Ap. J. 487:579

Ho LC, Filippenko AV, Sargent WLW. 1997d. Ap. J. 487:591

Ho LC, Filippenko AV, Sargent WLW. 2003. Ap. J. 583:159

Ho LC, Filippenko AV, Sargent WLW, Peng CY. 1997e. Ap. J. Suppl. 112:391

Ho LC, Peng CY. 2001. Ap. J. 555:650

Ho LC, Ptak A, Terashima Y, Kunieda H, Serlemitsos PJ, et al. 1999a. Ap. J. 525:168

Ho LC, Rudnick G, Rix H-W, Shields JC, McIntosh DH, et al. 2000. Ap. J. 541:120

Ho LC, Sarzi M, Rix H-W, Shields JC, Rudnick G, et al. 2002. PASP 114:137

Ho LC, Shields JC, Filippenko AV. 1993. Ap. J. 410:567

Ho LC, Terashima Y, Ulvestad JS. 2003. Ap. J. 589:783

Ho LC, Ulvestad JS. 2001. Ap. J. Suppl. 133:77

Ho LC, Van Dyk SD, Pooley GG, Sramek RA, Weiler KW. 1999b. Astron. J 118:843

Hopkins PF, Hernquist L. 2006. Ap. J. Suppl. 166:1

Hopkins PF, Hernquist L, Cox TJ, Di Matteo T, Martini P, et al. 2006. Ap. J. Suppl. $163: 1$

Huchra JP, Burg R. 1992. Ap. J. 393:90

Humason ML, Mayall NU, Sandage AR. 1956. Astron. J. 61:97

Igumenshchev IV, Narayan R, Abramowicz MA. 2003. Ap. J. 592:1042

Inoue H, Terashima Y, Ho LC. 2007. Ap. J. 662:860

Irwin JA, Sarazin CL, Bregman JN. 2002. Ap. J. 570:152

Ishisaki Y, Makishima K, Iyomoto N, Hayashida K, Inoue H, et al. 1996. PASJ 48:237

Iyomoto N, Fukazawa Y, Nakai N, Ishihara Y. 2001. Ap. J. 561:L69 
Iyomoto N, Makishima K, Fukazawa Y, Tashiro M, Ishisaki Y, et al. 1996. PASJ, $48: 231$

Iyomoto N, Makishima K, Fukazawa Y, Tashiro M, Ishisaki Y. 1997. PASJ 49:425

Iyomoto N, Makishima K, Matsushita K, Fukazawa Y, Tashiro M, Ohashi T. 1998a. Ap. J. 503:168

Iyomoto N, Makishima K, Tashiro M, Inoue S, Kaneda H, et al. 1998b. Ap. J. 503:L31

Jolley EJD, Kuncic Z. 2007. Ap\&SS 310:327

Johnson BM, Quataert E. 2007. Ap. J. 660:1273

Jungwiert B, Combes F, Palous J. 2001. Astron. Astrophys. 376:85

Kauffmann G, Heckman TM, Tremonti C, Brinchmann J, Charlot S, et al. 2003. MNRAS 346:1055

Kauffmann G, White SDM, Heckman TM, Meńard B, Brinchmann J, et al. 2004. MNRAS 353:713

Keel WC. 1983a. Ap. J. 268:632

Keel WC. 1983b. Ap. J. 269:466

Keel WC. 1983c. Ap. J. Suppl. 52:229

Keel WC, Miller JS. 1983. Ap. J. 266:L89

Kennicutt RC Jr. 1984. Ap. J. 287:116

Kewley LJ, Groves B, Kauffmann G, Heckman T. 2006. MNRAS 372:961

Kewley LJ, Heisler CA, Dopita MA, Lumsden S. 2001. Ap. J. Suppl. 132:37

Khachikian EY, Weedman DW. 1974. Ap. J. 192:581

Kharb P, Shastri P. 2004. Astron. Astrophys. 425:825

Kim D-C, Sanders DB, Veilleux S, Mazzarella JM, Soifer BT. 1995. Ap. J. Suppl. 98:129

Kim D-W, Fabbiano G. 2003. Ap. J. 586:826

Kinkhabwala A, Sako M, Behar E, Kahn SM, Paerels F, et al. 2002. Ap. J. 575:732

Kirhakos S, Phillips MM. 1989. PASP 101:949

Komossa S, Böhringer H, Huchra JP. 1999. Astron. Astrophys. 349:88

Koratkar AP, Deustua S, Heckman TM, Filippenko AV, Ho LC, Rao M. 1995. Ap. J. 440:132

Körding E, Falcke H, Corbel S. 2006. Astron. Astrophys. 456:439

Körding E, Jester S, Fender R. 2006. MNRAS 372:1366

Körding E, Jester S, Fender R. 2008. MNRAS 383:277

Kormendy J. 1993. In The Nearest Active Galaxies, ed. J Beckman, L Colina, H Netzer, p. 197. (Madrid: CSIC)

Kormendy J. 2004. In Carnegie Observatories Astrophysics Series, Vol 1: Coevolution of Black Holes and Galaxies, ed. LC Ho, p. 1. Cambridge: Cambridge Univ. Press

Kormendy J, Fisher DB, Cornell ME, Bender R. 2008. Ap. J. Suppl. submitted

Kormendy J, Kennicutt RC. 2004. Annu. Rev. Astron. Astrophys. 42:603

Kormendy J, Richstone DO. 1995. Annu. Rev. Astron. Astrophys. 33:581

Koski AT, Osterbrock DE. 1976. Ap. J. 203:L49

Krips M, Eckart A, Krichbaum TP, Pott J-U, Leon S, et al. 2007. Astron. Astrophys. 464:553

Krolik JH. 1998. Active Galactic Nuclei. Princeton: Princeton Univ. Press

Kukula MJ, Pedlar A, Baum SA, O'Dea CP. 1995. MNRAS 276:1262

Kunth D, Sargent WLW, Bothun GD. 1987. Astron. J. 92:29

Laor A. 2003. Ap. J. 590:86

Larkin JE, Armus L, Knop RA, Soifer BT, Matthews K. 1998. Ap. J. Suppl. 
114:59

Lasota J-P, Abramowicz MA, Chen X, Krolik J, Narayan R, Yi I. 1996. Ap. J. 462:142

Laurikainen E, Salo H, Buta R. 2004. Ap. J. 607:103

Lawrence A. 2005. MNRAS 363:57

Lawrence A, Elvis M. 1982. Ap. J. 256:410

Lawrence A, Ward M, Elvis M, Fabbiano G, Willner SP, et al. 1985. Ap. J. 291:117

Lewis KT, Eracleous M, Gliozzi M, Sambruna RM, Mushotzky RF. 2005. Ap. J. 622:816

Lewis KT, Eracleous M, Sambruna RM. 2003. Ap. J. 593:115

Li C, Kauffmann G, Heckman TM, White SDM, Jing YP. 2008. MNRAS in press Lightman AP, White TR. 1988. Ap. J. 335:57

Lin DC, Shields GA. 1986. Ap. J. 305:28

Lira P, Lawrence A, Johnson RA. 2000. MNRAS 319:17

Liu BF, Meyer-Hofmeister E. 2001. Astron. Astrophys. 372:386

Liu BF, Taam RE, Meyer-Hofmeister E, Meyer F. 2007. Ap. J. 671:695

Livio M, Ogilvie GI, Pringle JE. 1999. Ap. J. 512:100

Loewenstein M, Mushotzky RF, Angelini L, Arnaud KA, Quataert E. 2001. Ap. J. 555:L21

Maccarone TJ, Fender RP, Ho LC, eds. 2005. From X-ray Binaries to Quasars: Black Hole Accretion on All Mass Scales Dordrecht: Kluwer

Maccarone TJ, Gallo E, Fender RP. 2003. MNRAS 345:L19

Magorrian J, Tremaine S, Richstone D, Bender R, Bower G, et al. 1998. Astron. J. 115:2285

Mahadevan R. 1997. Ap. J. 477:585

Maia MAG, Machado RS, Willmer CNA. 2003. Astron. J. 126:1750

Maiolino R, Rieke GH. 1995. Ap. J. 454:95

Makishima K, Fujimoto R, Ishisaki Y, Kii T, Loewenstein M, et al. 1994. PASJ 46:L77

Makishima K, Ohashi T, Hayashida K, Inoue H, Koyama K, et al. 1989. PASJ 41:697

Malkan MA, Sargent WLW. 1982. Ap. J. 254:22

Maoz D. 2007. MNRAS 377:1696

Maoz D, Filippenko AV, Ho LC, Macchetto FD, Rix H-W, Schneider DP. 1996. Ap. J. Suppl. 107:215

Maoz D, Filippenko AV, Ho LC, Rix H-W, Bahcall JN, et al. 1995. Ap. J. 440:91

Maoz D, Koratkar AP, Shields JC, Ho LC, Filippenko AV, Sternberg A. 1998. Astron. J. 116:55

Maoz D, Nagar NM, Falcke H, Wilson AS. 2005. Ap. J. 625:699

Matsumoto Y, Fukazawa Y, Nakazawa K, Iyomoto N, Makishima K. 2001. PASJ $53: 475$

Meier DL. 1999. Ap. J. 522:753

Meier DL. 2001. Ap. J. 548:L9

Meisenheimer K, Tristram KRW, Jaffe W, Israel F, Neumayer N, et al. 2007. Astron. Astrophys. 471:453

Menou K, Quataert E. 2001. Ap. J. 552:204

Merloni A, Fabian AC. 2002. MNRAS 332:165

Merloni A, Heinz S, Di Matteo T. 2003. MNRAS 345:1057

Merloni A, Nayakshin S, Sunyaev R, eds. 2005. Growing Black Holes: Accretion 
in a Cosmological Context Berlin: Springer-Verlag

Middleton M, Done C, Schurch N. 2008. MNRAS 383:1501

Miller CJ, Nichol RC, Gomez PL, Hopkins AM, Bernardi M. 2003. Ap. J. 597:142

Milosavljević M, Merritt D, Ho LC. 2006. Ap. J. 652:120

Minkowski R, Osterbrock DE. 1959. Ap. J. 129:583

Miniutti G, Ponti G, Greene JE, Ho LC, Fabian AC, Iwasawa K. 2008. MNRAS in press

Moore B, Katz N, Lake G, Dressler A, Oemler A. 1996. Nature 379:613

Moran EC, Eracleous M, Leighly KM, Chartas G, Filippenko AV, et al. 2005. Astron. J. 129:2108

Müller SAH, Haas M, Siebenmorgen R, Klaas U, Meisenheimer K, et al. 2004. Astron. Astrophys. 426:L29

Muno MP, Baganoff FK, Bautz MW, Feigelson ED, Garmire GP, et al. 2004. Ap. J. $613: 326$

Murray N, Chiang J. 1997. Ap. J. 474:91

Mushotzky RF. 1993. In The Nearest Active Galaxies, ed. J Beckman, L Colina, H Netzer, p. 47. Madrid: CSIC Press

Mushotzky RF, Wandel A. 1989. Ap. J. 339:674

Nagao T, Murayama T, Shioya Y, Taniguchi Y. 2002. Ap. J. 567:73

Nagar NM, Falcke H, Wilson AS. 2005. Ap. J. 435:521

Nagar NM, Falcke H, Wilson AS, Ho LC. 2000. Ap. J. 542:186

Nagar NM, Falcke H, Wilson AS, Ulvestad JS. 2002. Astron. Astrophys. 392:53

Nagar NM, Wilson AS, Falcke H. 2001. Ap. J. 559:L87

Nandra K, George IM, Mushotzky RF, Turner TJ, Yaqoob T. 1997a. Ap. J. 476:70

Nandra K, George IM, Mushotzky RF, Turner TJ, Yaqoob T. 1997b. Ap. J. 477:602

Nandra K, O'Neill PM, George IM, Reeves JN. 2007. MNRAS 382:194

Narayan R. 2002. In Lighthouses of the Universe, ed. M Gilfanov et al., p. 405. Berlin: Springer

Narayan R, Yi I. 1995. Ap. J. 444:231

Nayakshin S. 2003. Astron. Nachr. Suppl. 324:3

Nelson CH, Whittle M. 1996. Ap. J. 465:96

Nemmen RS, Storchi-Bergmann T, Yuan F, Eracleous M, Terashima Y, Wilson AS. 2006. Ap. J. 643:652

Nicastro F. 2000. Ap. J. 530:L65

Nicholson KL, Reichert GA, Mason KO, Puchnarewicz EM, Ho LC, et al. 1998. MNRAS 300:893

Noyola E, Gebhardt K, Bergmann M. 2008. Ap. J. in press

O'Connell RW. 1999. Annu. Rev. Astron. Astrophys. 37:603

O'Connell RW, Martin JR, Crane JD, Burstein D, Bohlin RC, et al. 2005. Ap. J. $635: 305$

Omma H, Binney J, Bryan G, Slyz A. 2004. MNRAS 348:1105

Osterbrock DE. 1960. Ap. J. 132:325

Osterbrock DE. 1971. In Nuclei of Galaxies, ed. DJK O'Connell, p. 151. Amsterdam: North Holland

Osterbrock DE, Dufour RJ. 1973. Ap. J. 185:441

Osterbrock DE, Miller JS. 1975. Ap. J. 197:535

Osterbrock DE, Shaw RA. 1988. Ap. J. 327:89

Padovani P, Matteucci F. 1993. Ap. J. 416:26 
Page MJ, Breeveld AA, Soria R, Wu K, Branduardi-Raymont G, et al. 2003. Astron. Astrophys. 400:145

Page MJ, Soria R, Zane S, Wu K, Starling R. 2004. Astron. Astrophys. 422:77

Panessa F, Barcons X, Bassani L, Cappi M, Carrera FJ, et al. 2007. Astron. Astrophys. 467:519

Panessa F, Bassani L. 2002. Astron. Astrophys. 394:435

Panessa F, Bassani L, Cappi M, Dadina M, Barcons X, et al. 2006. Astron. Astrophys. 455:173

Pappa A, Georgantopoulos I, Stewart GC, Zezas AL. 2001. MNRAS 326:995

Peimbert M, Torres-Peimbert S. 1981. Ap. J. 245:845

Pelat D, Alloin D, Fosbury RAE. 1981. MNRAS 195:787

Pellegrini S. 2005. Ap. J. 624:155

Pellegrini S, Baldi A, Fabbiano G, Kim D-W. 2003a. Ap. J. 597:175

Pellegrini S, Cappi M, Bassani L, Della Ceca R, Palumbo GGC. 2000a. Astron. Astrophys. 360:878

Pellegrini S, Cappi M, Bassani L, Malaguti G, Palumbo GGC, Persic M. 2000b. Astron. Astrophys. 353:447

Pellegrini S, Fabbiano G, Fiore F, Trinchieri G, Antonelli A. 2002. Astron. Astrophys. 383:1

Pellegrini S, Siemiginowska A, Fabbiano G, Elvis M, Greenhill L, et al. 2007. Ap. J. $667: 749$

Pellegrini S, Venturi T, Comastri A, Fabbiano G, Fiore F, et al. 2003b. Ap. J. 585:677

Peng CY, Ho LC, Impey CD, Rix H-W. 2002. Astron. J. 124:266

Penston MV, Fosbury RAE. 1978. MNRAS 183:479

Péquignot D. 1984. Astron. Astrophys. 131:159

Peterson BM, Bentz MC, Desroches L-B, Filippenko AV, Ho LC, et al. 2005. Ap. J. 632:799. Erratum. 2005. Ap. J. 641:638

Petre R, Mushotzky RF, Serlemitsos PJ, Jahoda K, Marshall FE. 1993. Ap. J. 418:644

Phillips MM. 1979. Ap. J. 227:L121

Phillips MM, Charles PA, Baldwin JA. 1983. Ap. J. 266:485

Phillips MM, Jenkins CR, Dopita MA, Sadler EM, Binette L. 1986. Astron. J. 91:1062

Pogge RW. 1989. Ap. J. Suppl. 71:433

Pogge RW, Maoz D, Ho LC, Eracleous M. 2000. Ap. J. 532:323

Proga D, Begelman MC. 2003. Ap. J. 592:767

Ptak A, Serlemitsos PJ, Yaqoob T, Mushotzky R. 1999. Ap. J. Suppl. 120:179

Ptak A, Terashima Y, Ho LC, Quataert E. 2004. Ap. J. 606:173

Ptak A, Yaqoob T, Mushotzky R, Serlemitsos P, Griffiths R. 1998. Ap. J. 501:L37

Ptak A, Yaqoob T, Serlemitsos PJ, Kunieda H, Terashima Y. 1996. Ap. J. 459:542 Quataert E. 2003. Astron. Nachr. Suppl. 324:435

Quataert E, Di Matteo T, Narayan R, Ho LC. 1999. Ap. J. 525:L89

Quillen AC, McDonald C, Alonso-Herrero A, Lee A, Shaked S, et al. 2001. Ap. J. $547: 129$

Ravindranath S, Ho LC, Peng CY, Filippenko AV, Sargent WLW. 2001. Astron. J. 122:653

Rees MJ, Phinney ES, Begelman MC, Blandford RD. 1982. Nature 295:17

Reeves JN, Turner MJL. 2000. MNRAS 316:234

Renzini A, Greggio L, di Serego Alighieri S, Cappellari M, Burstein D, Bertola 
F. 1995. Nature 378:39

Reynolds CS, Di Matteo T, Fabian AC, Hwang U, Canizares CR. 1996. MNRAS 283:L111

Rice MS, Martini P, Greene JE, Pogge RW, Shields JC, et al. 2006. Ap. J. 636:654

Richstone DO. 2004. In Carnegie Observatories Astrophysics Series, Vol 1: Coevolution of Black Holes and Galaxies, ed. LC Ho, p. 280. Cambridge: Cambridge Univ. Press

Rieke GH, Lebofsky MJ, Kemp JC. 1982. Ap. J. 252:L53

Rinn AS, Sambruna RM, Gliozzi M. 2005. Ap. J. 621:167

Roberts TP, Schurch NJ, Warwick RS. 2001. MNRAS 324:737

Roberts TP, Warwick RS. 2000. MNRAS 315:98

Rola C, Terlevich E, Terlevich R. 1997. MNRAS 289:419

Rose JA, Searle L. 1982. Ap. J. 253:556

Rose JA, Tripicco MJ. 1984. Ap. J. 285:55

Rubin VC, Ford WK Jr, Thonnard N. 1980. Ap. J. 238:471

Rupke D, Veilleux S, Kim D-C, Sturm E, Contursi A, et al. 2007. In The Central Engine of Active Galactic Nuclei, ed. LC Ho, J-M Wang, p. 525. San Francisco: ASP

Sabra BM, Shields JC, Ho LC, Barth AJ, Filippenko AV 2003. Ap. J. 584:164

Sadler EM, Jenkins CR, Kotanyi CG. 1989. MNRAS 240:591

Sambruna RM, Gliozzi M, Eracleous M, Brandt WN, Mushotzky RF. 2003. Ap. J. 586:L37

Sandage A, Bedke J. 1994. The Carnegie Atlas of Galaxies. Washington, DC: Carnegie Inst. of Washington

Sandage A, Tammann, GA. 1981. A Revised Shapley-Ames Catalog of Bright Galaxies. Washington, DC: Carnegie Inst. of Washington

Sargent WLW, Filippenko AV. 1991. Astron. J. 102:107

Sarzi M, Shields JC, Pogge RW, Martini P. 2007. In The Central Engine of Active Galactic Nuclei, ed. LC Ho, J-M Wang, p. 643. San Francisco: ASP

Sarzi M, Rix H-W, Shields JC, Ho LC, Barth AJ, et al. 2005. Ap. J. 628:169

Satyapal S, Dudik RP, O'Halloran B, Gliozzi M. 2005. Ap. J. 633:86

Satyapal S, Sambruna RM, Dudik RP. 2004. Astron. Astrophys. 414:825

Satyapal S, Vega D, Dudik RP, Abel NP, Heckman T. 2008. Ap. J. in press

Satyapal S, Vega D, Heckman T, O'Halloran B, Dudik R. 2007. Ap. J. 663:L9

Schmidt M, Green RF. 1983. Ap. J. 269:352

Schmitt HR. 2001. Astron. J. 122:2243

Schmitt HR, Kinney AL, Ho LC, eds. 1999. The AGN/Normal Galaxy Connection Oxford: Elsevier Science Ltd.

Schulz H, Fritsch C. 1994. Astron. Astrophys. 291:713

Serote-Roos M, Gonçalves AC. 2004. Astron. Astrophys. 413:91

Seth AC, Agüeros M, Lee D, Basu-Zych A. 2008. Ap. J. in press

Shakura NI, Sunyaev RA. 1973. Astron. Astrophys. 24:337

Shang Z, Brotherton MS, Green RF, Kriss GA, Scott J, et al. 2005. Ap. J. 619:41

Shields GA. 1978. Nature. 272:706

Shields GA, Wheeler JC. 1978. Ap. J. 222:667

Shields JC. 1992. Ap. J. 399:L27

Shields JC, Rix H-W, McIntosh DH, Ho LC, Rudnick G, et al. 2000. Ap. J. 534:L27

Shields JC, Rix H-W, Sarzi M, Barth AJ, Filippenko AV, et al. 2007. Ap. J. 654:125 
Shields JC, Sabra BM, Ho LC, Barth AJ, Filippenko AV. 2002. In Mass Outflow in Active Galactic Nuclei: New Perspectives, ed. DM Crenshaw, SB Kraemer, IM George, p. 105. San Francisco: ASP

Shields JC, Walcher CJ, Böker, T, Ho LC, Rix H-W, van der Marel RP. 2008. $A p$. $J$. in press

Shih DC, Iwasawa K, Fabian AC. 2003. MNRAS 341:973

Siemiginowska A, Czerny B, Kostyunin V. 1996. Ap. J. 458:491

Sikora M, Stawarz L, Lasota J-P. 2007. Ap. J. 658:815

Slee OB, Sadler EM, Reynolds JE, Ekers RD. 1994. MNRAS 269:928

Smith JDT, Draine BT, Dale DA, Moustakas J, Kennicutt RC, et al. 2007. Ap. J. 656:770

Soltan A. 1982. MNRAS 200:115

Soria R, Fabbiano G, Graham A, Baldi A, Elvis M, et al. 2006. Ap. J. 640:126

Spinoglio L, Malkan MA. 1992. Ap. J. 399:504

Springel V, Di Matteo T, Hernquist L. 2005. MNRAS 361:776

Starling RLC, Page MJ, Branduardi-Raymont G, Breeveld AA, Soria R, Wu K. 2005. MNRAS 356:727

Stasińska G. 1984. Astron. Astrophys. 135:341

Stasińska G, Cid Fernandes R, Mateus A, Sodré L Jr, Asari NV. 2006. MNRAS 371:972

Stauffer JR. 1982a. Ap. J. Suppl. 50:517

Stauffer JR. 1982b. Ap. J. 262:66

Stauffer JR, Spinrad H. 1979. Ap. J. 231:L51

Stone JD, Pringle JE. 2001. MNRAS 322:461

Storchi-Bergmann T, Baldwin JA, Wilson AS. 1993. Ap. J. 410:L11

Storchi-Bergmann T, Ho LC, Schmitt HR, eds. 2004. IAU Symp. 222, Interplay among Black Holes, Stars and ISM in Galactic Nuclei Cambridge: Cambridge Univ. Press

Storchi-Bergmann T, Pastoriza MG. 1990. PASP 102:1359

Strateva IV, Brandt WN, Schneider DP, Vanden Berk DG, Vignali C. 2005. Astron. J. 130:387

Sturm E, Rupke D, Contursi A, Kim D-C, Lutz D, et al. 2006. Ap. J. 653:L13

Sturm E, Schweitzer M, Lutz D, Contursi A, Genzel R, et al. 2005. Ap. J. 629:L21

Sugai H, Malkan MA. 2000. Ap. J. 529:219

Sulentic JW, Marziani P, Dultzin-Hacyan D. 2000. Annu. Rev. Astron. Astrophys. $38: 521$

Swartz DA, Ghosh KK, Suleimanov V, Tennant AF, Wu K. 2002. Ap. J. 574:382

Szokoly GP, Bergeron J, Hasinger G, Lehmann I, Kewley L, et al. 2004. Ap. J. Suppl. 155:271

Tan JC, Blackman EG. 2005. MNRAS 362:983

Taniguchi Y, Shioya Y, Murayama T. 2000. Astron. J. 120:1265

Terashima Y, Ho LC, Ptak AF. 2000. Ap. J. 539:161

Terashima Y, Ho LC, Ptak AF, Mushotzky RF, Serlemitsos PJ, et al. 2000a. Ap. J. 533:729

Terashima Y, Ho LC, Ptak AF, Yaqoob T, Kunieda H, et al. 2000b. Ap. J. 535:L79

Terashima Y, Iyomoto N, Ho LC, Ptak AF. 2002. Ap. J. Suppl. 139:1

Terashima Y, Kunieda H, Misaki K, Mushotzky RF, Ptak AF, Reichert GA. 1998a. Ap. J. 503:212

Terashima Y, Ptak A, Fujimoto R, Itoh M, Kunieda H, et al. 1998b. Ap. J. 
496:210

Terashima Y, Wilson AS. 2003a. Ap. J. 560:139

Terashima Y, Wilson AS. 2003b. Ap. J. 583:145

Terlevich R, Melnick J. 1985. MNRAS 213:841

Tran HD. 2001. Ap. J. 554:L19

Tremaine S, Gebhardt K, Bender R, Bower G, Dressler A, et al. 2002. Ap. J. $574: 740$

Tremonti CA, Heckman TM, Kauffmann G, Brinchmann J, Charlot S, et al. 2004. Ap. J. 613:898

Turner TJ, Pounds KA. 1989. MNRAS 240:833

Tzanavaris P, Georgantopoulos I. 2007. Astron. Astrophys. 468:129

Ulvestad JS, Greene JE, Ho LC. 2007. Ap. J. 661:L159

Ulvestad JS, Ho LC. 2001a. Ap. J. 558:561

Ulvestad JS, Ho LC. 2001b. Ap. J. 562:L133

Ulvestad JS, Ho LC. 2002. Ap. J. 581:925

Ulvestad JS, Wilson AS. 1989. Ap. J. 343:659

Vanden Berk DE, Richards GT, Bauer A, Strauss MA, Schneider DP, et al. 2001. Astron. J. 122:549

Van Dyk SD, Ho LC. 1998. In IAU Symp. 184, The Central Regions of the Galaxy and Galaxies, ed. Y Sofue, p. 489. Dordrecht: Kluwer

Varano S, Chiaberge M, Macchetto FD, Capetti A. 2004. Astron. Astrophys. 428:401

Veilleux S, Osterbrock DE. 1987. Ap. J. Suppl. 63:295

Verdoes Kleijn GA, Baum SA, de Zeeuw PT, O'Dea CP. 2002. Astron. J. 123:1334

Verdoes Kleijn GA, van der Marel RP, Noel-Storr J. 2006. Astron. J. 131:1961

Véron P, Gonçalves AC, Véron-Cetty M-P. 1997. Astron. Astrophys. 319:52

Véron P, Véron-Cetty M-P. 1986. Astron. Astrophys. 161:145

Véron-Cetty M-P, Véron P. 1986. Astron. Astrophys. Suppl. 66:335

Véron-Cetty M-P, Véron P. 2006. Astron. Astrophys. 455:773

Viegas-Aldrovandi SM, Gruenwald RB. 1990. Ap. J. 360:474

Voit GM. 1992. Ap. J. 399:495

Voit GM, Donahue M. 1997. Ap. J. 486:242

Wake DA, Miller CJ, Di Matteo T, Nichol RC, Pope A, et al. 2004. Ap. J. 610:L85

Walcher CJ, Böker T, Charlot S, Ho LC, Rix H-W, et al. 2006. Ap. J. 649:692

Walsh JL, Barth AJ, Ho LC, Filippenko AV, Rix H-W, et al. 2008. Ap. J. submitted

Wang J-M, Luo B, Ho LC. 2004. Ap. J. 615:L5

Wang J-M, Staubert R, Ho LC. 2002. Ap. J. 579:554

Weaver KA, Wilson AS, Henkel C, Braatz JA. 1999. Ap. J. 520:130

Weedman DW, Feldman FR, Balzano VA, Ramsey LW, Sramek RA, Wu C-C. 1981. Ap. J. 248:105

Whysong D, Antonucci R. 2004. Ap. J. 602:116

Willner SP, Ashby MLN, Barmby P, Fazio GG, Pahre M, et al. 2004. Ap. J. Suppl. 154:222

Willner SP, Elvis M, Fabbiano G, Lawrence A, Ward MJ. 1985. Ap. J. 299:443

Wilson AS 1979. Proc. Roy. Soc. London A366:461

Worrall DM, Birkinshaw M. 1994. Ap. J. 427:134

Woźniak PR, Zdziarski AA, Smith D, Madejski GM, Johnson WN. 1998. MNRAS 299:449

Wrobel JM. 1991. Astron. J. 101:127 
Wrobel JM, Fassnacht CD, Ho LC. 2001. Ap. J. 553:L23

Wrobel JM, Heeschen DS. 1991. Astron. J. 101:148

Wrobel JM, Ho LC. 2006. Ap. J. 646:L95

Wrobel JM, Terashima Y, Ho LC. 2008. Ap. J. in press

Wu Q, Cao X. 2005. Ap. J. 621:130

Wu Q, Yuan F, Cao X. 2007. Ap. J. 669:96

$\mathrm{Xu}$ Y, Cao X-W. 2007. ChJAA 7:63

Yan R, Newman JA, Faber SM, Konidaris N, Koo D, Davis M. 2006. Ap. J. 648:281

Yaqoob T, Serlemitsos PJ, Ptak A, Mushotzky RF, Kunieda H, Terashima Y. 1995. Ap. J. 455:508

Young AJ, Nowak MA, Markoff S, Marshall HL, Canizares CR. 2007. Ap. J. 669:830

Yu Q, Tremaine S. 2002. MNRAS 335:965

Yuan F. 2007. In The Central Engine of Active Galactic Nuclei, ed. LC Ho, J-M Wang, p. 95. San Francisco: ASP

Yuan F, Cui W. 2005. Ap. J. 629:408

Yuan F, Markoff S, Falcke H. 2002. Astron. Astrophys. 383:854

Yuan F, Markoff S, Falcke H, Biermann PL. 2002. Astron. Astrophys. 391:139

Yuan F, Narayan R. 2004. Ap. J. 612:724

Zakamska NL, Strauss MA, Krolik JH, Collinge MJ, Hall PB, et al. 2003. Astron. J. 126:2125

Zamorani G, Henry JP, Maccacaro T, Tananbaum H, Sołtan A, et al. 1981. Ap. J. $245: 357$

Zezas A, Birkinshaw M, Worrall DM, Peters A, Fabbiano G. 2005. Ap. J. 627:711

Zhang X-G, Dultzin-Hacyan D, Wang T-G. 2007. MNRAS 374:691

Zhang Y, Gu Q-S, Ho LC. 2008. Astron. Astrophys. submitted 